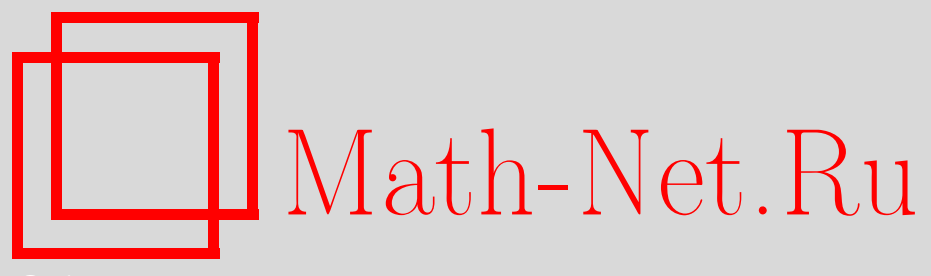

А. Д. Коршунов, Сложность вычислений булевых функций, УМН, 2012, том 67, выпуск 1,97-168

DOI: https://doi.org/10.4213/rm9459

Использование Общероссийского математического портала Math-Net.Ru подразумевает, что вы прочитали и согласны с пользовательским соглашением http://www . mathnet.ru/rus/agreement

Параметры загрузки:

IP: 18.234 .197 .8

26 апреля 2023 г., 13:02:29

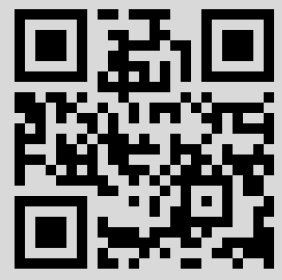




\title{
Сложность вычислений булевых функций
}

\begin{abstract}
А. Д. Коршунов
Булевы функции являются одним из основных объектов дискретной математики, в особенности тех ее разделов, которые входят в математическую логику и математическую кибернетику. Язык булевых функций удобен для описания функционирования многих дискретных систем, например, контактных схем, булевых схем, ветвящихся программ и некоторых других. Важным параметром таких дискретных систем является их сложность. Эта характеристика активно изучается, начиная с работ К. Шеннона. Опубликовано много научных статей, в которых содержится большое число результатов. Цель обзора - изложение основных результатов по сложности вычислений (реализации) булевых функций контактными схемами, булевыми схемами и булевыми схемами без ветвлений, которые получены за последние шестьдесят лет.

Библиография: 165 названий.

Ключевые слова: базис, булевы схемы, булева функция, глубина и задержка булевой схемы, дизъюнктивная нормальная форма, инвариантные классы булевых функций, клеточная схема, контактная схема без нулевых цепей, логическая формула, нижние оценки сложности схем, параллельно-последовательная контактная схема, симметрическая булева функция, сложность схемы, частичная булева функция.
\end{abstract}

\section{СОДЕРЖАНИЕ}

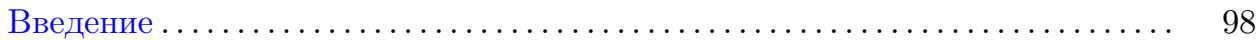

1. Контактные схемы . . . . . . . . . . . . . . . . . . . . . . . . 101

1.1. Общие све́дения о контактных схемах ................... 101

1.2. Произвольные контактные схемы . . . . . . . . . . . . . . . . 102

1.3. Параллельно-последовательные контактные схемы . . . . . . . . . . 107

1.4. Плоские, локально-неплоские и клеточные контактные схемы . . 110

1.5. Контактные схемы без нулевых цепей . . . . . . . . . . . . . . . . 112

1.6. Контактные схемы ограниченной степени .............. 113

Работа выполнена при поддержке Российского фонда фундаментальных исследований (проект № 08-01-00611) и Программы фундаментальных исследований ОМН РАН “Алгебраические и комбинаторные методы математической кибернетики" (проект "Новые методы дискретной и комбинаторной оптимизации").

(C) А. Д. Коршунов, 2012 
1.7. Метод Храпченко для $\pi$-схем ........................ 114

1.8. Симметрические булевы функции . . . . . . . . . . . . . . . 115

1.9. Инвариантные классы булевых функций .................. 116

1.10. Нижние оценки сложности минимальных контактных схем . . . 117

1.11. Дизъюнктивные нормальные формы ..................... 119

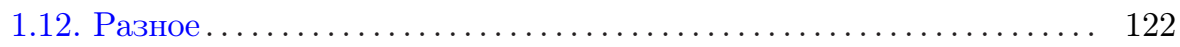

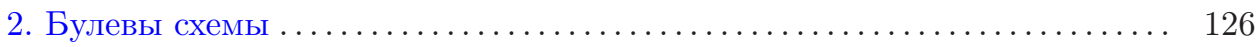

2.1. Общие све́дения о булевых схемах ..................... 126

2.2. Произвольные булевы схемы . . . . . . . . . . . . . . . . 126

2.3. Булевы схемы с ограниченным ветвлением ................ 130

2.4. Метод локального кодирования Лупанова ................... 131

2.5. Частичные булевы функции . . . . . . . . . . . . . . . . . . . 133

2.6. Схемы из пороговых элементов ...................... 134

2.7. Булевы схемы в бесконечных базисах ................... 136

2.8. Глубина булевых схем . . . . . . . . . . . . . . . . . . . . . . 137

2.9. Булевы схемы с задержками ....................... 138

2.10. Активность булевых схем . . . . . . . . . . . . . . . . . . . . . 139

2.11. Неявная и параметрическая выразимость булевых функций ... 140

2.12. Клеточные булевы схемы . . . . . . . . . . . . . . . . . 142

2.13. Булевы схемы в базисах, содержащих элементы с нулевыми

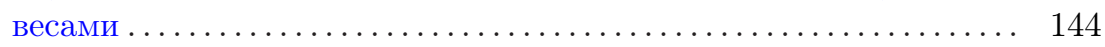

2.14. Полиномиальные нижние оценки сложности булевых схем, реализующих конкретные булевы функции . . . . . . . . . . 145

2.15. Высокие нижние оценки сложности булевых схем. Методы Разборова и Андреева................................... 146

2.16. Разное ..................................... 150

3. Булевы схемы без ветвлений . . . . . . . . . . . . . . . . . . 152

3.1. Общие све́дения о булевых схемах без ветвлений .............. 152

3.2. Сложность булевых схем без ветвлений..................... 154

3.3. Булевы схемы без ветвлений в базисах, содержащих элементы с нулевыми весами . . . . . . . . . . . . . . . . . . . . 156

3.4. Глубина и сложность булевых схем без ветвлений ........... 156

3.5. Нижние оценки сложности булевых схем без ветвлений, вычисляющих конкретные булевы функции ................ 157

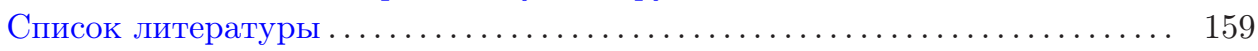

\section{Введение}

Булева функция - функция, переменные которой, как и сама функция, принимают значения 0 и 1 . Булевы функции сочетают предельную простоту с универсальностью.

Булевы функции являются одним из основных объектов дискретной математики, в особенности тех ее разделов, которые входят в математическую логику и математическую кибернетику. Они стали использоваться при изучении логических задач и названы по имени Дж. Буля, положившего начало их применению в математической логике. 
Позднее выяснилось, что язык булевых функций удобен для описания функционирования некоторых дискретных систем: контактные схемы, булевы схемы (частный вид схем из функциональных элементов), формулы (суперпозиции, булевы схемы без ветвлений) и др. Наиболее известными из них стали булевы схемы и булевы схемы без ветвлений.

Следует также заметить, что многие задачи общей теории вычислительной сложности без ограничения общности можно представить как задачу вычисления последовательности булевых функций от $n$ переменных при различных $n$.

Из сказанного следует, что исследование сложности вычислений булевых функций является весьма актуальным.

При изучении сложности вычислений булевых функций различного вида схемами естественными мерами сложности являются такие, как число контактов в контактной схеме, число элементов в булевой схеме, занимаемый ею объем, число конъюнкций в кратчайшей дизъюнктивной нормальной форме булевой функции и т. п.

Как правило, существует тривиальное решение задачи о построении схем минимальной сложности. Например, при построении схемы с минимальным числом элементов (контактов, элементов, реализующих предписанные булевы функции, и т. п.) тривиальный метод решения состоит в переборе сначала схем, содержащих один элемент, с проверкой того, есть ли среди них схема, вычисляющая заданную функцию, затем в переборе схем с двумя элементами и т. д. до тех пор, пока не встретится схема, вычисляющая заданную функцию. Эта схема является искомой.

Из-за большого числа таких шагов метод перебора схем практически неосуществим. Поэтому требуется уточнение задачи синтеза схем.

Опубликовано большое число статей, в которых исследованы различные аспекты сложности вычислений булевых функций. Отдельные результаты освещены в монографиях и учебных пособиях [1]-[11] и в обзорных статьях [12]-[18].

Однако до сих пор нет публикации, в которой достаточно полно были бы изложены основные результаты исследований по сложности вычислений булевых функций контактными и контактно-вентильными схемами, булевыми схемами (схемами из функциональных элементов), бинарными программами и некоторыми схемами других видов, полученные начиная с работ К. Шеннона.

Цель настоящего обзора состоит в освещении основных результатов, относящихся к сложности вычисления булевых функций контактными схемами, булевыми схемами и булевыми схемами без ветвлений, которые получены за последние шестьдесят лет. При этом следует отметить, что обзор является в основном обзором по максимальной сложности вычисления булевых функций (эффект Шеннона-Лупанова) с включением некоторых результатов по сложности вычисления индивидуальных булевых функций.

Отметим, что в обзоре отсутствует следующая информация:

1) све́дения о сложности вычислений многозначных функций;

2) поскольку размер обзора ограничен, в списке литературы по существу отсутствуют сведения о статьях, опубликованных в журнале "Доклады АН CCСР", диссертациях, отчетах, тезисах и статьях в электронном виде; 
3) информация о работах, в которых рассматриваются вычисления булевых функций без анализа сложности получаемых схем; как правило, такие работы пишут инженеры, специалисты по проектированию вычислительной техники и т. д.;

4) информация о сложности вычислений монотонных булевых функций (за исключением нескольких случаев), поскольку основные результаты, относящиеся к этой тематике, освещены в [18];

5) результаты по диагностике и синтезу надежных схем; число публикаций по этой тематике велико и для их освещения нужен отдельный обзор;

6) све́дения о сложности вычислений булевых функций ветвящимися бинарными программами; по этой тематике имеется несколько сот публикаций, в том числе монографии и обзорные статьи; требуется написание подробного обзора.

На протяжении всего обзора используются следующие понятия и обозначения.

Число единиц в двоичном наборе называется весом набора. Через $E^{n}$ обозначается $n$-мерный булев куб, т. е. неориентированный граф с $2^{n}$ вершинами, которые помечены двоичными наборами длины $n$. В этом графе две вершины, помеченные наборами $\widetilde{\alpha}=\left(\alpha_{1}, \ldots, \alpha_{n}\right)$ и $\widetilde{\beta}=\left(\beta_{1}, \ldots, \beta_{n}\right)$, смежны (соединены ребром) тогда и только тогда, когда расстояние Хемминга $\rho(\widetilde{\alpha}, \widetilde{\beta})=\sum_{i=1}^{n}\left|\alpha_{i}-\beta_{i}\right|$ равно 1. Множество вершин в $E^{n}$, помеченных наборами веса $k, 0 \leqslant k \leqslant n$, называется $k$-м слоем в $E^{n}$ и обозначается через $E^{n, k}$.

Через $\lfloor x\rfloor$ обозначается наибольшее целое число, не превосходящее действительное число $x$, а через $\lceil x\rceil$ - минимальное целое число, не меньшее $x$.

Пусть функции $f(n)$ и $g(n)$ принимают положительные значения при любом натуральном $n$. Тогда запись $f(n)=O(g(n))$ (или $f(n) \leqslant O(g(n)))$ означает: существует такая константа $c>0$, что $f(n)<c g(n)$ при любом $n \geqslant 1$. Запись $f(n)=\Theta(g(n))$ означает: существуют константы $c_{1}, c_{2}>0$ такие, что $c_{1} g(n) \leqslant f(n) \leqslant c_{2} g(n)$ при любом $n \geqslant 1$. Запись $f(n) \sim g(n)$ означает, что $\lim _{n \rightarrow \infty}(f(n) / g(n))=1$. Запись $f(n)=\Omega(g(n))$ означает существование такой константы $c>0$, что $f(n) \geqslant c g(n)$ при любом $n \geqslant 1$.

Пусть $\widetilde{\alpha}=\left(\alpha_{1}, \ldots, \alpha_{n}\right)$ и $\widetilde{\beta}=\left(\beta_{1}, \ldots, \beta_{n}\right)$ - различные двоичные наборы длины $n$. Говорят, что набор $\widetilde{\alpha}$ предшествует набору $\widetilde{\beta}$ ( $\widetilde{\alpha}$ меньше $\widetilde{\beta}$ ) (обозначение: $\widetilde{\alpha} \prec \widetilde{\beta})$, если $\alpha_{i} \leqslant \beta_{i}$ при любом $i, 1 \leqslant i \leqslant n$.

Булева функция $f\left(x_{1}, \ldots, x_{n}\right)$ называется монотонной, если $f(\widetilde{\alpha}) \leqslant f(\widetilde{\beta})$ на любых наборах $\widetilde{\alpha}=\left(\alpha_{1}, \ldots, \alpha_{n}\right), \widetilde{\beta}=\left(\beta_{1}, \ldots, \beta_{n}\right)$ длины $n$ таких, что $\widetilde{\alpha} \prec \widetilde{\beta}$.

Литералом называется булева переменная или ее отрицание. Элементарной конгюнкиией называется логическое произведение различных литералов, в котором отсутствуют литералы вида $x$ и $\bar{x}$. В случае, когда число множителей равно 0, конъюнкция называется пустой.

Пусть $\sigma$ равно либо 0, либо 1. Для обозначения литералов используется функция $x^{\sigma}$, определяемая следующим образом:

$$
x^{\sigma}= \begin{cases}x & \text { при } \sigma=1, \\ \bar{x} & \text { при } \sigma=0 .\end{cases}
$$


Ясно, что $x^{\sigma}=1$ тогда и только тогда, когда $x=\sigma$, т. е. значение "основания" равно значению "показателя".

Пусть $P$ - произвольное свойство булевых функций, $B_{n}-$ множество некоторых булевых функций от переменных $x_{1}, \ldots, x_{n}$ такое, что $\left|B_{n}\right| \rightarrow \infty$ при $n \rightarrow \infty$, $P_{B}(n)$ - множество функций из $B_{n}$, обладающих свойством $P$. Говорят, что почти все функции из $B_{n}$ обладают свойством $P$, если $\lim _{n \rightarrow \infty}\left|P_{B}(n)\right| /\left|B_{n}\right|=1$.

\section{1. Контактные схемы}

1.1. Общие све́дения о контактных схемах. Контактные схемы являются удобной математической моделью электромеханических релейно-контактных устройств, которые доминировали в доэлектронную эпоху в автоматике и телемеханике. Они определяются следующим образом.

Контактной схемой называется связный неориентированный граф (без петель и, возможно, с параллельными ребрами) с несколькими выделенными вершинами (называемыми полюсами), каждому ребру которого приписана одна из букв $x_{1}, \ldots, x_{n}, \bar{x}_{1}, \ldots, \bar{x}_{n}$ (разным ребрам может быть приписана одна и та же буква), где $x_{1}, \ldots, x_{n}$ являются булевыми переменными, а $\bar{x}_{1}, \ldots, \bar{x}_{n}$ - их отрицаниями.

Ребро с приписанной ему переменной $x_{i}$ (или $\bar{x}_{i}$ ) называется замыкающим (размыкающим) контактом. Последовательность контактов между двумя полюсами $a$ и $b$ схемы $S$, соответствующая простой цепи в графе схемы $S$, называется иепъю между полюсами $a$ и $b$ схемы $S$; конъюнкция соответствующих букв (литералов) называется проводимостью цепи.

Цепь между полюсами $a$ и $b$ в контактной схеме $S$ замкнута, если замкнуты все ее элементы, и разомкнута, если разомкнут хотя бы один ее контакт. Пример контактной схемы с двумя полюсами $a$ и $b$ приведен на рис. 1.1.

Пусть $C$ - произвольная цепь, соединяющая полюсы $a$ и $b$. Если цепь состоит из контактов $x_{i_{1}}^{\sigma_{1}}, x_{i_{2}}^{\sigma_{2}}, \ldots, x_{i_{l}}^{\sigma_{l}}$, то ей сопоставляется конъюнкция $K_{l}=$ $x_{i_{1}}^{\sigma_{1}} \& x_{i_{2}}^{\sigma_{2}} \& \cdots \& x_{i_{l}}^{\sigma_{l}}$. Например, цепи рис. 1.1 , проходящей через вершины $a, c$, $f, b$, соответствует конъюнкция $x \bar{y} \bar{z}$.

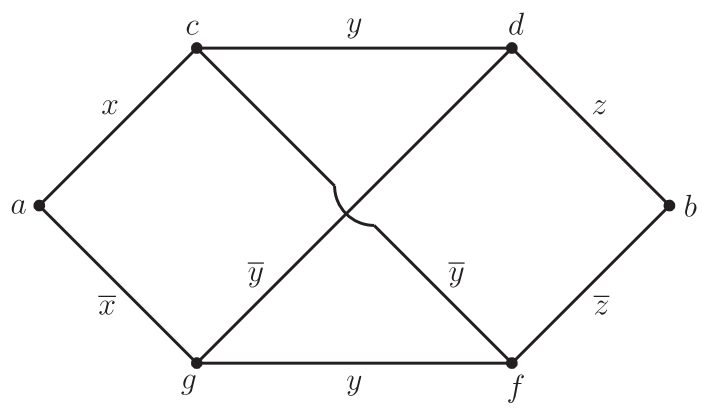

Рис. 1.1

Дизъюнкция проводимостей всех замкнутых цепей между полюсами $a$ и $b$ схемы $S$ называется проводимостью схемы $S$ между полюсами $a$ и $b$. 
Всякая булева функция реализуема многими двухполюсными контактными схемами.

Иногда в контактной схеме множество всех полюсов разбивается на непересекающиеся подмножества входов и выходов. Контактная схема с $r$ входами и $s$ выходами называется контактным $(r, s)$-полюсником. В статье в основном используются контактные $(1,1)$-полюсники и $(1, s)$-полюсники при $s \geqslant 2$.

Контактная схема, в которой проводимости между любыми двумя выходами равны 0, называется разделительной (по выходам).

Контактная схема называется ориентированной, если граф такой схемы является ориентированным. В ориентированной контактной схеме каждый контакт имеет одностороннюю проводимость.

Двухполюсной контактно-вентильной схемой называется сеть без петель с двумя выделенными вершинами (полюсами) и множеством ребер и дуг. Каждое ребро интерпретируется как контакт с приписанной буквой из конечного множества $\left\{x_{1}, \ldots, x_{n}, \bar{x}_{1}, \ldots, \bar{x}_{n}\right\}$, а каждая дуга, ориентированная от вершины $u$ к вершине $v$, интерпретируется как вентиль, проводящий в направлении от $u$ к $v$.

Один полюс такой схемы называется входным, а другой - выходным полюсом схемы. Простая цепь от входного полюса $a$ к выходному полюсу $b$ в контактно-вентильной схеме $S$ замкнута, если замкнуты все ее контакты, и каждый вентиль, содержащийся в этой цепи, ориентирован от $a$ к $b$.

Проводимостью в схеме $S$ от полюса $a$ к полюсу $b$ называется совокупность всех замкнутых простых цепей от $a$ и $b$. Таким образом, проводимость от полюса $a$ к полюсу $b$ в $S$ задается булевой функцией от тех переменных, которые присутствуют в $S$.

1.2. Произвольные контактные схемы. Задача построения схем с минимальным числом контактов (иначе, схем с минимальной сложностью), реализующих булевы функции из заданного множества, рассматривается с сороковых годов прошлого века. Имеется тривиальный метод построения таких экстремальных схем, основанный на переборе всех схем ограниченной сложности, реализующих заданную функцию, и выбора из них схемы с минимальным числом контактов. Однако такой метод построения схем, как правило, мало эффективен, поскольку требует перебора очень большого числа схем.

Среди известных методов построения (контактных и других) схем имеются универсальные методы и методы синтеза схем, вычисляющих конкретные функции.

Задача об отыскании универсальных методов синтеза контактных схем может быть сформулирована следующим образом. Пусть $\mathscr{F}$ - некоторый класс булевых функций (например, всех булевых функций) и $\Sigma$ - множество контактных схем, которые можно использовать для вычисления функций из $\mathscr{F}$. Предполагается, что каждая функция из $\mathscr{F}$ может быть вычислена по меньшей мере одной схемой из $\Sigma$.

Через $L_{\Sigma}(f)$ обозначается нижняя грань сложности схем из $\Sigma$, вычисляющих функцию $f$ из $\mathscr{F}$, а через $L_{\Sigma}^{\mathscr{F}}(n)$ - наибольшее из чисел $L_{\Sigma}(f)$, где максимум берется по всем функциям $f$ из $\mathscr{F}$ от $n$ переменных. Требуется найти такой 
“эффективный” метод синтеза схем из $\Sigma$, который для любой функции $f$ из $\mathscr{F}$ от $n$ переменных позволяет строить такую схему $S$ из $\Sigma$, что $S$ вычисляет $f$ и имеет не более $L_{\Sigma}^{\mathscr{F}}(n)\left(1+\varepsilon_{n}\right)$ контактов, где $\varepsilon_{n} \rightarrow \infty$ при $n \rightarrow \infty$, либо $\varepsilon_{n}=$ const.

Функция $L_{\Sigma}^{\mathscr{F}}(n)$ впервые введена в рассмотрение К. Шенноном при исследовании сложности минимальных контактных схем, реализующих произвольные булевы функции [19], [20]. Впоследствии такие функции стали называть сложностными функииями Шеннона.

Пусть $f$ - произвольная булева функция. Обозначим через $L_{k}(f)$ минимальное число контактов, достаточное для построения контактной схемы, реализующей функцию $f$, и пусть $L_{k}(n)=\max L_{k}(f)$, где максимум берется по всем булевым функциям от $n$ переменных.

Первые серьезные исследования по синтезу контактных схем провел К. Шеннон. В работе [20] им доказана следующая теорема.

ТеОРема 1.1. При любом фиксированном $\varepsilon>0 u n \rightarrow \infty$

$$
\frac{2^{n}}{n}\left(1-\frac{\log _{2} n}{n}\right)<L_{k}(n)<\frac{2^{n+2}}{n}(1+\varepsilon),
$$

причем среди булевых функиий от переменных $x_{1}, \ldots, x_{n}$ доля функиий $f$ таких, что $L_{k}(f)<\frac{2^{n}}{n}\left(1-\frac{\log _{2} n}{n}\right)$, стремится $\kappa$ нулю с ростом $n$.

Доказательства нижней и верхней оценок для $L_{k}(n)$ основаны на использовании разных идей. Нижняя оценка устанавливается с применением следующего мощностного соображения. Пользуясь верхней оценкой для числа двухполюсных контактных схем с заданным числом контактов, нетрудно убедиться в том, что число схем, в каждой из которых содержится не более $\left\lfloor\frac{2^{n}}{n}\left(1-\frac{\log _{2} n}{n}\right)\right\rfloor$ контактов, меньше числа всех булевых функций от переменных $x_{1}, \ldots, x_{n}$, т. е. величины $2^{2^{n}}$. Отсюда следует нижняя оценка для $L_{k}(n)$ из теоремы 1.1 .

Верхняя оценка извлекается из предложенного метода синтеза контактных схем, который для каждой булевой функции от $n$ переменных позволяет строить реализующую ее схему не более чем с $\left[\frac{2^{n+2}}{n}(1+\varepsilon)\right\rfloor$ контактами.

Позднее в [21], [22] Г.Н. Поваров предложил усовершенствование метода Шеннона - так называемый метод каскадов, позволяющий для отдельных булевых функций получить простые схемы. Однако метод каскадов не позволяет понизить верхнюю оценку для $L_{k}(n)$ из теоремы 1.1 .

Окончательное решение задачи об асимптотическом поведении функции $L_{k}(n)$ получено О.Б. Лупановым в [23]. Им был предложен такой метод построения контактных схем, который для каждой булевой функции $f$ от $n$ переменных позволяет получить схему, реализующую $f$ и имеющую не более $\left\lfloor\frac{2^{n}}{n}\left(1+O\left(\frac{1}{\sqrt{n}}\right)\right)\right\rfloor$ контактов. Этот результат формулируется следующим образом. 


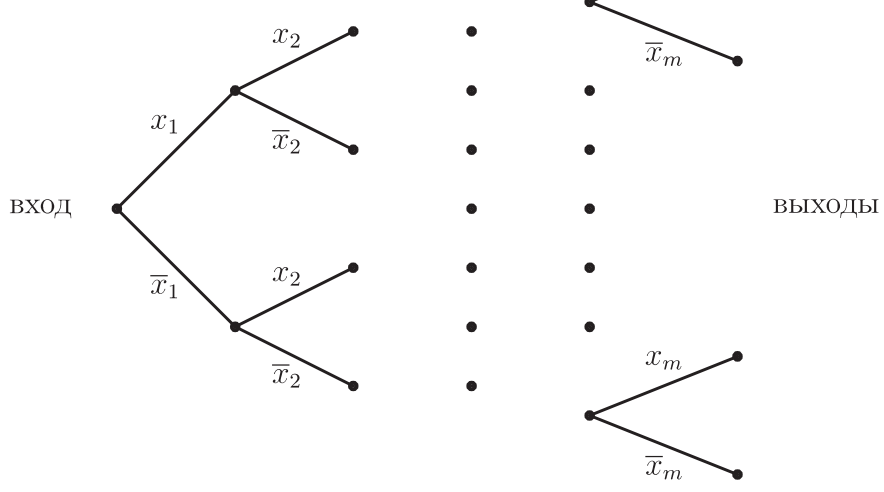

Рис. 1.2

TeOpema 1.2. При $n \rightarrow \infty$

$$
L_{k}(n)<\frac{2^{n}}{n}\left(1+O\left(\frac{1}{\sqrt{n}}\right)\right) .
$$

Одновременно О.Б. Лупанов получил следующую более точную (нежели в теореме 1.1) нижнюю оценку для $L_{k}(n)$.

ТеОрема 1.3. Если $\varepsilon>0$ - произвольное число и $n \rightarrow \infty$, то среди булевых функиий от переменных $x_{1}, \ldots, x_{n}$ доля функиий $f$ таких, что

$$
L_{k}(f)<\frac{2^{n}}{n}\left(1+(2-\varepsilon) \frac{\log _{2} n}{n}\right),
$$

стремится к нулю с ростом $n$.

Из теорем 1.2 и 1.3 вытекает следующая теорема.

TeOpema 1.4. При $n \rightarrow \infty$

$$
L_{k}(f) \sim \frac{2^{n}}{n},
$$

причем при любом $\varepsilon>0$ среди булевых функиий от переменных $x_{1}, \ldots, x_{n}$ доля функций $f$ таких, что $L_{k}(f)=\frac{2^{n}}{n}\left(1+(2-\varepsilon) \frac{\log _{2} n}{n}\right)$, стремится к нулю с ростом $n$.

Предложенный О. Б. Лупановым метод синтеза контактных схем основан на специальном задании булевых функций и на использовании оригинального контактного $\left(1,2^{m}\right)$-полюсника ( $m$ подбирается по $\left.n\right)$, реализующего все элементарные конъюнкции длины $m$ от переменных $x_{1}, \ldots, x_{m}$ и являющегося асимптотически минимальным по числу контактов. Этот $\left(1,2^{m}\right)$-полюсник (дешифратор на $m$ переменных) в два раза более экономен, чем обычное контактное дерево на $m$ переменных. 
Обычное контактное дерево, реализующее все элементарные конъюнкции длины $m$ от переменных $x_{1}, \ldots, x_{m}$, имеет один входной полюс (вход) и $2^{m}$ выходных полюсов (выходов) (см. рис. 1.2). Каждая элементарная конъюнкция длины $n$ реализуется в этом дереве между входом и соответствующим выходом. Легко видеть, что в таком дереве содержится $2^{m+1}-2$ контакта. Это дерево обладает следующим свойством разделительности: каждая цепь, соединяющая любые два выхода, содержит замыкающий и размыкающий контакты одного переменного и поэтому имеет нулевую проводимость. Следовательно, если в этом дереве объединить некоторые выходы, то между входом и полученным выходом будет реализовываться дизъюнкция соответствующих элементарных конъюнкций.

Большинство инженеров, работавших в то время в области построения контактных схем, предполагали, что контактное дерево, реализующее все элементарные конъюнкции от заданного числа переменных, содержит минимально возможное число контактов. Однако это мнение оказалось ошибочным, что следует из дешифратора Лупанова. Вместе с тем обычное контактное дерево на $m$ переменных содержит минимальное число контактов в классе всех разделительных $\left(1,2^{m}\right)$-полюсников. Этот факт установлен Э. Муром [24].

Контактный дешифратор Лупанова, не являясь разделительным, обладает некоторым "ослабленным" свойством разделительности. Это свойство позволяет использовать такой дешифратор при синтезе контактных схем и получить асимптотику для $L_{k}(n)$.

Описанный О.Б. Лупановым дешифратор на $m$ переменных содержит не более $2^{m}(1+\Omega(1 / m))$ контактов. $\mathrm{K}$ этому направлению относится работа автора [25], в которой изучалась следующая задача.

Два контакта $x$ и $\bar{x}$, соединенные так, как указано на рис. 1.3 , называются вилкой. Контактный $(1, k)$-полюсник $A$ называется $(1, k)$-полюсником из переключательных контактов, если множество контактов в $A$ можно разбить на систему попарно непересекающихся (по контактам) вилок.

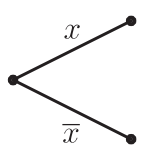

Рис. 1.3

Автор настоящего обзора в [25] установил, что число контактов в минимальном $(1, k)$-полюснике из переключательных контактов, реализующем произвольную систему $k$ попарно ортогональных булевых функций, не меньше $2 k-2$.

Пусть $\mathscr{K}_{1}, \ldots, \mathscr{K}_{m_{n}}$ - совокупность различных элементарных конъюнкций длины $n$ от переменных $x_{1}, \ldots, x_{n}$, где $m_{n}<2^{n}$. Обозначим через $L_{k}\left(\mathscr{K}_{1}, \ldots, \mathscr{K}_{m_{n}}\right)$ минимальное число контактов, достаточное для построения контактного $\left(1, m_{n}\right)$ полюсника, реализующего конъюнкции $\mathscr{K}_{1}, \ldots, \mathscr{K}_{m_{n}}$. Пусть

$$
L_{k}^{1}\left(n, m_{n}\right)=\max L_{k}\left(\mathscr{K}_{1}, \ldots, \mathscr{K}_{m_{n}}\right),
$$


где максимум берется по всем совокупностям $m_{n}$ различных элементарных конъюнкций длины $n$ от переменных $x_{1}, \ldots, x_{n}$. Далее, пусть $L_{k}^{2}\left(n, m_{n}\right)=$ $\max L_{k}(f)$, где $L_{k}(f)$ определена выше, а максимум берется по булевым функциям от $n$ переменных, принимающим значение 1 на $m_{n}$ наборах из $E^{n}$.

В работе [26] Н. П. Редькин установил следующие факты.

Теорема 1.5. Пусть последовательность $m_{1}, m_{2}, \ldots$ такова, что $\log _{2} n=$ $o\left(\log _{2} m_{n}\right) u \log _{2} m_{n}=o(n)$. Тогда

$$
L_{k}^{1}\left(n, m_{n}\right) \sim L_{k}^{2}\left(n, m_{n}\right) \sim \frac{n m_{n}}{\log _{2} m_{n}} .
$$

ТЕОРема 1.6. Если при целом $r>1$ выполнено условие $r \log _{2} m_{n} \sim n$, то

$$
L_{k}^{2}\left(n, m_{n}\right) \sim(r-1) \log _{2} m_{n} .
$$

Сложность реализации системы всех булевых функций от переменных $x_{1}, \ldots, x_{n}$ контактными $\left(1,2^{2^{n}}\right)$-полюсниками изучали С. А. Ложкин и М. А. Кошкин. В [27] они опубликовали следующий результат.

Теорема 1.7. Число контактов в минимальном $\left(1,2^{2^{n}}\right)$-полюснике, реализующем все булевы функиии от переменных $x_{1}, \ldots, x_{n}$, асимптотически равно $2 \cdot 2^{2^{n}}$.

Кроме теоремы 1.7, в указанной работе установлены асимптотики для числа контактов в минимальных многополюсниках, реализующих другие системы булевых функций.

Теперь рассмотрим контактные схемы ограниченной ширины.

Сечением в двухполюсной контактной схеме $S$ называется множество контактов в $S$, которое содержит хотя бы по одному контакту из каждой цепи схемы $S$.

Сечение называется тупиковым (несужаемым), если любое его собственное подмножество не является сечением. Шириной двухполюсной контактной схемы называется число контактов в максимальном (по мощности) тупиковом сечении этой схемы.

Пусть $f$ - произвольная булева функция. Обозначим через $L_{k}(f, b)$ число контактов в минимальной контактной схеме ширины $b$, которая реализует функцию $f$, и пусть $L_{k}(n, b)=\max L_{k}(f, b)$, где максимум берется по всем булевым функциям $f$ от $n$ переменных.

Х. А. Мадатян в [28] доказал следующее утверждение.

Теорема 1.8. Пусть $b$ maково, что $n^{\alpha(n)} \leqslant b \leqslant 2^{n} / n$, где $\alpha(n) \rightarrow \infty$ npu $n \rightarrow \infty$. Тогда

$$
L_{k}(n, b) \sim \frac{2^{n}}{\log _{2}(n b)} .
$$

Наряду с обычными контактными схемами рассматриваются ориентированные контактные схемы. Такие схемы отличаются от обычных контактных схем только тем, что каждый контакт схемы проводит только в одном направлении. 
Обозначим через $\vec{L}_{k}(f)$ число контактов в минимальной (по числу контактов) ориентированной контактной схеме, реализующей булеву функцию $f$. Пусть $\vec{L}_{k}(n)=\max \vec{L}_{k}(f)$, где максимум берется по всем булевым функциям от $n$ переменных. Используя мощностные соображения, можно убедиться в том, что при $n \rightarrow \infty$

$$
\vec{L}_{k}(n) \geqslant \frac{2^{n}}{n}\left(1+\frac{2 \log _{2} n}{n}-\frac{c_{1}}{n}+o\left(\frac{1}{n}\right)\right),
$$

где $c_{1}$ - подходящая положительная константа.

Вместе с тем С.А. Ложкин в [29] доказал, что при $n \rightarrow \infty$

$$
\vec{L}_{k}(n) \leqslant \frac{2^{n}}{n}\left(1+\frac{2 \log _{2} n}{n}-\frac{c_{2}}{n}+o\left(\frac{1}{n}\right)\right),
$$

где $c_{2}$ - подходящая положительная константа.

Таким образом, верхняя (1.2) и нижняя (1.1) оценки функции $\vec{L}_{k}(n)$ различаются на величину $O\left(2^{n} / n^{2}\right)$. Поэтому при $n \rightarrow \infty$

$$
\vec{L}_{k}(n) \sim \frac{2^{n}}{n} .
$$

1.3. Параллельно-последовательные контактные схемы. Среди контактных схем самостоятельный интерес представляют параллельно-последовательные контактные схемы, которые часто называют $\pi$-схемами. Эти схемы можно определить индуктивно следующим образом:

1) один контакт есть $\pi$-схема;

$2)$ если $S_{1}\left(a_{1}, b_{1}\right)$ и $S_{2}\left(a_{2}, b_{2}\right)$ суть $\pi$-схемы, то схема $S(a, b)$, полученная отождествлением (склеиванием) полюса $a_{1}$ с $a_{2}$ и полюса $b_{1}$ с $b_{2}$, есть $\pi$-схема с полюсами $a=a_{1}=a_{2}$ и $b=b_{1}=b_{2}$;

$3)$ схема $S\left(a_{1}, b_{2}\right)$, полученная склеиванием полюса $b_{1}$ с $a_{2}$, есть $\pi$-схема (последовательное соединение $\pi$-схем $S_{1}\left(a_{1}, b_{1}\right)$ и $\left.S_{2}\left(a_{2}, b_{2}\right)\right)$ с полюсами $a_{1}$ и $b_{2}$;

4) других $\pi$-схем нет.

Пусть $L_{\pi}(f)$ есть число контактов в минимальной $\pi$-схеме, реализующей булеву функцию $f$, и $L_{\pi}(n)=\max L_{\pi}(f)$, где максимум берется по всем булевым функциям от $n$ переменных. Дж. Риордан и К. Шеннон в [30] доказали следующее утверждение.

TeOpema 1.9. При $n \rightarrow \infty$

$$
L_{\pi}(n)>\frac{2^{n}}{\log _{2} n}\left(1-\frac{3}{\log _{2} n}\right),
$$

причем среди булевых функиий $f$ от переменных $x_{1}, \ldots, x_{n}$ доля таких функиий $f$, что $L_{\pi}(f)<\frac{2^{n}}{\log _{2} n}\left(1-\frac{3}{\log _{2} n}\right)$, стремится $\kappa$ нулю с ростом $n$. 
Верхние оценки для $L_{\pi}(n)$ были получены О. Б. Лупановым. Сначала в [31] им был предложен такой метод синтеза $\pi$-схем, который позволяет получать $\pi$-схемы, реализующие булевы функции от $n$ переменных, в каждой из которых содержится не более $\left\lfloor\frac{2^{n+1}}{\log _{2} n}\left(1+\varepsilon_{n}\right)\right\rfloor$ контактов, где $\varepsilon_{n} \rightarrow 0$ при $n \rightarrow \infty$. Впоследствии О. Б. Лупанов в [32] описал другой метод синтеза $\pi$-схем, из которого извлекается следующая теорема.

TEOPEMA 1.10. При $n \rightarrow \infty$

$$
L_{\pi}(n)<\frac{2^{n}}{\log _{2} n}\left(1+O\left(\frac{\log _{2} \log _{2} n}{\log _{2} n}\right)\right) .
$$

Из теорем 1.9 и 1.10 вытекает следующая теорема.

TEOPEMA 1.11. При $n \rightarrow \infty$

$$
L_{\pi}(n) \sim \frac{2^{n}}{\log _{2} n},
$$

причем среди булевых функииц $f$ от переменных $x_{1}, \ldots, x_{n}$ доля таких функций $f$, что $L_{\pi}(f)<\frac{2^{n}}{\log _{2} n}\left(1-\frac{3}{\log _{2} n}\right)$, стремится $к$ нулю с ростом $n$.

Дадим индуктивное определение ранга $\pi$-схемы. Контактную $\pi$-схему назовем дизгюнктивной $\pi$-схемой, если она состоит из двух параллельно соединенных $\pi$-схем. Контактную $\pi$-схему назовем конгюнктивной $\pi$-схемой, если она состоит из двух последовательно соединенных $\pi$-схем.

Контактная схема, состоящая из одного контакта, имеет ранг 1.

Если $\pi$-схема $S$ состоит из параллельно соединенных дизъюнктивных $\pi$-схем $S_{1}$ и $S_{2}$ ранга $r_{1}$ и $r_{2}$ соответственно, то ранг схемы $S$ равен $\max \left\{r_{1}, r_{2}\right\}$.

Если $\pi$-схема $S$ состоит из последовательно соединенных конъюнктивных $\pi$-схем $S_{1}$ и $S_{2}$ ранга $r_{1}$ и $r_{2}$ соответственно, то ранг схемы $S$ равен $\max \left\{r_{1}, r_{2}\right\}$.

Если $\pi$-схема $S$ состоит из параллельно соединенных конъюнктивных $\pi$-схем $S_{1}, \ldots, S_{k}$ ранга $r_{1}, \ldots, r_{k}$ соответственно, то ранг схемы $S$ равен $1+$ $\max \left\{r_{1}, \ldots, r_{k}\right\}$.

Если $\pi$-схема $S$ состоит из последовательно соединенных дизъюнктивных $\pi$-схем $S_{1}, \ldots, S_{k}$ ранга $r_{1}, \ldots, r_{k}$ соответственно, то ранг схемы $S$ равен $1+$ $\max \left\{r_{1}, \ldots, r_{k}\right\}$.

Ранг контактной $\pi$-схемы часто называют глубиной. Однако это название неудачно, поскольку оно используется в другом смысле для булевых схем (см. п. 2.12).

Пусть $L_{\pi}^{r}(f)$ обозначает число контактов в минимальной $\pi$-схеме ранга не более $r$, реализующей булеву функцию $f$, и пусть $L_{\pi}^{r}(n)=\max L_{\pi}^{r}(f)$, где максимум берется по всем булевым функциям от $n$ переменных.

Легко видеть, что $L_{\pi}^{2}(n) \geqslant L_{\pi}^{3}(n) \geqslant \cdots$.

О. Б. Лупанов в [33] доказал следующее утверждение. 
Теорема 1.12. При каждом фиксированном $r \geqslant 3 u n \rightarrow \infty$

$$
L_{\pi}^{r}(n) \sim \frac{2^{n}}{\log _{2} n},
$$

причем при любом фиксированном $\varepsilon>0$ среди булевых функиий от переменных $x_{1}, \ldots, x_{n}$ доля функций $f$ таких, что $L_{\pi}^{r}(f)<\frac{2^{n}}{\log _{2} n}(1-\varepsilon)$, стремится $\kappa$ нулю с ростом $n$.

Этот результат в определенном смысле является окончательным, поскольку при любом $n \geqslant 2$

$$
L_{\pi}^{2}(n)=n \cdot 2^{n-1} .
$$

Двухполюсная сеть $S$ (параллельно-последовательная сеть $S$ ) называется $n$-универсальной, если для любой булевой функции $f\left(x_{1}, \ldots, x_{n}\right)$ возможна расстановка по ребрам сети $S$ литералов $x_{1}, \ldots, x_{n}, \bar{x}_{1}, \ldots, \bar{x}_{n}$ (быть может с повторениями; некоторые литералы могут не использоваться), превращающая $S$ в двухполюсную контактную схему (параллельно-последовательную схему), реализующую функцию $f\left(x_{1}, \ldots, x_{n}\right)$.

Пусть $U(n)\left(U_{\pi}^{r}(n)\right)$ обозначает число ребер в минимальной (по числу ребер) $n$-универсальной сети ( $n$-универсальной параллельно-последовательной сети ранга $r)$.

Ясно, что если в двухполюсной схеме $S$ имеется $k$ ребер, то в результате приписывания литералов всем ребрам получается $(2 n)^{k}$ контактных схем. Так как должно выполняться неравенство $(2 n)^{k} \geqslant 2^{2^{n}}$, то $U(n)>\frac{2^{n}}{1+\log _{2} n}$.

Е. А. Кондратьева в [34] доказала следующее утверждение.

TEOPEMA 1.13. При $n \rightarrow \infty$

$$
U_{\pi}^{7}(n) \sim \frac{2^{n}}{\log _{2} n}
$$

Поскольку $U(n) \leqslant U_{\pi}^{7}(n)$ и $U(n)>\frac{2^{n}}{1+\log _{2} n}$, из теоремы 1.13 следует, что

$$
U(n) \sim \frac{2^{n}}{\log _{2} n}
$$

ЗАмечАниЕ. В работе [34] имеется неточность. Эта неточность исправлена в [35].

Позднее результат Е. А. Кондратьевой улучшил О. Б. Лупанов. В работе [36] он установил следующее утверждение, являющееся усилением теоремы 1.12.

TEOPEMA 1.14. При $n \rightarrow \infty$

$$
U_{\pi}^{3}(n) \sim \frac{2^{n}}{\log _{2} n} .
$$


Этот результат является окончательным, поскольку в классе $\pi$-схем ранга 2 линейная булева функция $f\left(x_{1}, \ldots, x_{n}\right)=x_{1}+\cdots+x_{n}(\bmod 2)$ имеет сложность $n \cdot 2^{n-1}$.

К этому направлению относится работа Е. В. Шеришевой [37], в которой изучается сложность минимальных контактных схем, являющихся универсальными в следующем смысле.

Двухполюсная контактная сеть $S$ называется универсальной для всех булевых функций от переменных $x_{1}, \ldots, x_{n}$, если подстановкой констант 0 и 1 вместо некоторых контактов можно получить схему, реализующую любую наперед заданную булеву функцию от переменных $x_{1}, \ldots, x_{n}$.

Пусть $\mathscr{A}_{\pi}(n)\left(\mathscr{A}_{\pi}^{r}(n)\right)$ обозначает число контактов в минимальной универсальной $\pi$-схеме (минимальной универсальной $\pi$-схеме ранга $r$ ) для всех булевых функций от переменных $x_{1}, \ldots, x_{n}$.

В [37] Е. В. Шеришева установила следующие результаты:

$$
\mathscr{A}_{\pi}(n) \sim 2^{n}, \quad \mathscr{A}_{\pi}^{2}(n)=(n+1) \cdot 2^{n-1}, \quad \mathscr{A}_{\pi}^{3}(n) \leqslant 2^{n+1} \log _{2} n .
$$

\section{4. Плоские, локально-неплоские и клеточные контактные схемы.} Контактная схема называется плоской, если она получена из плоской сети заменой каждого ребра на контакт. Очевидно, каждая $\pi$-схема является плоской. Следовательно, класс двухполюсных плоских контактных схем шире класса двухполюсных $\pi$-схем. Поэтому возникает вопрос о соотношении сложностной функции Шеннона для плоских контактных схем, реализующих все булевы функции от $n$ переменных, и аналогичной функции Шеннона в классе $\pi$-схем.

Оказывается, что при $n \rightarrow \infty$ асимптотическое поведение этих функций одинаково. Действительно, Ф.Я. Ветухновский в [38] показал, что число двухполюсных плоских контактных схем не более чем с $k$ контактами не превосходит величины $(c k)^{n}$, где $c$ - подходящая константа. Очевидно, что неравенство $(c k)^{n} \geqslant 2^{2^{n}}$ может выполняться только при

$$
k \geqslant \frac{2^{n}}{\log _{2}(c n)}=\frac{2^{n}}{\log _{2} n}\left(1-\Omega\left(\frac{1}{\log _{2} n}\right)\right) .
$$

Вместе с тем из теоремы 1.10 следует, что при $n \rightarrow \infty$ рассматриваемая функция Шеннона не превосходит величины $\frac{2^{n}}{\log _{2} n}(1+o(1))$. Следовательно, она асиптотически равна $\frac{2^{n}}{\log _{2} n}$ (ср. с теоремой 1.11).

Теперь рассмотрим локально-неплоские контактные схемы, определяемые следующим образом.

Пусть $\varphi(n)$ - произвольная целочисленная функция такая, что $1 \leqslant \varphi(n) \leqslant$ $2^{n} / n$, и $S$ - произвольная двухполюсная неориентированная плоская сеть без петель. В сети $S$ каждое ребро, инцидентное вершинам $v_{i}$ и $v_{j}$, заменяется на произвольную двухполюсную сеть $S^{\prime}$ без петель, содержащую не более $\varphi(n)$ ребер. При этом полюсы сети $S^{\prime}$ отождествляются с вершинами $v_{i}$ и $v_{j}$. Затем каждому ребру полученной сети приписывается произвольный контакт из множества $\left\{x_{1}, \ldots, x_{n}, \bar{x}_{1}, \ldots, \bar{x}_{n}\right\}$. В результате получается двухполюсная контактная схема, которая, вообще говоря, не является плоской. Эта схема реализует булеву функцию от $n$ переменных. 
Функция $\varphi(n)$ называется характеристикой контактной схемы.

Обозначим через $L(n, \varphi(n))$ минимальное число контактов, достаточное для построения локально-неплоской контактной схемы характеристики $\varphi(n)$, peaлизующей произвольную булеву функцию от $n$ переменных. В [39] доказано следующее утверждение.

ТеОрема 1.15. Пусть функиия $\varphi(n)$ удовлетворяет условию $1 \leqslant \varphi(n) \leqslant$ $2^{n} / n$. Тогда

$$
L(n, \varphi(n)) \sim \frac{2^{n}}{\log _{2} n+\log _{2} \varphi(n)} .
$$

Наконец, рассмотрим сложность реализации булевых функций клеточными контактными схемами. Среди клеточных контактных схем различают плоские и объемные (т. е. трехмерные). Наибольшее внимание уделяется плоским клеточным контактным схемам. Ниже будут рассматриваться только такие схемы. Их можно определить следующим образом.

Пусть плоский прямоугольник с целочисленными сторонами разбит на единичные квадраты (ячейки). Каждой ячейке приписывается один из символов следующего алфавита $\left\{x_{1}, \ldots, x_{n}, \bar{x}_{1}, \ldots, \bar{x}_{n} ; J, W ; A, B, C, \ldots\right\}$, причем каждый из символов $A, B, C, \ldots$ может быть приписан не более чем одной ячейке. Такой прямоугольник называют клеточной контактной схемой.

Ячейки, помеченные символами $x_{i}, 1 \leqslant i \leqslant n$, называются замыкающими контактами, символами $\bar{x}_{i}, 1 \leqslant i \leqslant n,-$ размыкающими контактами, символами $A, B, C, \ldots$ - полюсами (они располагаются на границе прямоугольника), символом $J$ - изоляторами, символом $W$ - проводниками (на рисунках для наглядности изоляторы изображают пустыми квадратами, а проводники - заштрихованными).

Цепъю называется такое множество ячеек $\left\{a_{1}, \ldots, a_{s}\right\}$, что для каждого $i$, $1 \leqslant i \leqslant s-1$, ячейки $a_{i}$ и $a_{i+1}$ имеют общую границу и среди них нет изоляторов.

Определим функционирование схемы $S$. Каждой цепи $Z$, которая соединяет два полюса, сопоставляется такая конъюнкция $K_{Z}$, что если $Z$ состоит только из проводников, то $K_{Z} \equiv 0$; в противном случае $K_{Z}$ равна конъюнкции литералов, присутствующих в цепи $Z$. Каждой паре полюсов, например, полюсам $A$ и $B$, схемы ставится в соответствие булева функция $f_{A B}\left(x_{1}, \ldots, x_{n}\right)=\bigvee K_{Z}$, где дизъюнкция берется по всем цепям, соединяющим $A$ и $B$. Если же полюсы $A$ и $B$ нельзя соединить цепью, то полагается $f_{A B}\left(x_{1}, \ldots, x_{n}\right) \equiv 0$.

Под сложностъю схемы $S$ понимается площадь прямоугольника, в котором она построена, т. е. число элементов в схеме $S$. Сложность схемы $S$ обозначается через $L_{k}^{\mathrm{c}}(S)$.

Пусть $f$ - произвольная булева функция, и пусть $L_{k}^{\mathrm{c}}(f)=\min L_{k}^{\mathrm{c}}(S)$, где минимум берется по всем двухполюсным клеточным контактным схемам, реализующих функцию $f$. Далее, пусть $L_{k}^{\mathrm{c}}(n)=\max L_{k}^{\mathrm{c}}(f)$, где максимум берется по всем булевым функциям от $n$ переменных. 
Ю. Г. Таразевич в [40] доказал, что при любом фиксированном $\varepsilon>0$ и $n \rightarrow \infty$

$$
\frac{2^{n}}{\log _{2} 36}(1-\varepsilon)<L_{k}^{\mathrm{c}}(n)<2^{n} .
$$

1.5. Контактные схемы без нулевых цепей. Цепь в контактной схеме, имеющая тождественно нулевую проводимость, называется нулевой цепью. Нулевая цепь обязательно содержит контакты $x$ и $\bar{x}$ некоторой переменной $x$.

Контактная схема называется контактной схемой без нулевых иепей, если любая ее простая цепь, соединяющая полюсы схемы, не является нулевой.

Класс контактных схем без нулевых цепей ввел в рассмотрение А. К. Пулатов [41]. Такие схемы изучали С. В. Здобнов [42]-[45], С. Е. Кузнецов [46], [47], И. О. Соколов [48].

В работе [41] А. К. Пулатовым была описана такая последовательность булевых функций $f\left(x_{1}, \ldots, x_{n}\right), n=1,2, \ldots$, что отношение числа контактов в минимальной контактной схеме без нулевых цепей, реализующей функцию $f\left(x_{1}, \ldots, x_{n}\right)$, к числу контактов в минимальной контактной схеме произвольного вида, реализующей ту же функцию, растет с ростом $n$. Аналогичное утверждение справедливо и для $\pi$-схем. Более точно, в этой работе получены следующие результаты.

Пусть $L_{k}(f)$ (соответственно $\left.L_{k}^{*}(f)\right)$ обозначает число контактов в минимальной контактной схеме произвольного вида (соответственно в минимальной контактной схеме без нулевых цепей), реализующей булеву функцию $f\left(x_{1}, \ldots, x_{n}\right)$, и пусть

$$
\mu_{k}(n)=\max \frac{L_{k}^{*}\left(f\left(x_{1}, \ldots, x_{n}\right)\right)}{L_{k}\left(f\left(x_{1}, \ldots, x_{n}\right)\right)}
$$

где максимум берется по всем булевым функциям от $n$ переменных. Аналогично определяется функция $\mu_{\pi}(n)$ в классе параллельно-последовательных контактных схем.

TeOPEмA 1.16. Пусть $\varepsilon_{n}>0 u \varepsilon_{n} \rightarrow 0$ npu $n \rightarrow \infty$. Тогда

$$
\mu_{k}(n)>\frac{n}{4 \log _{2} n}\left(1-\varepsilon_{n}\right), \quad \mu_{\pi}(n)>\frac{n}{4 \log _{2}^{2} n \cdot \log _{2} \log _{2} n}\left(1-\varepsilon_{n}\right) .
$$

Исследования по сложности контактных схем без нулевых цепей были продолжены С.Е. Кузнецовым. В работе [47] им был получен следующий результат. Пусть $L_{k}^{*}(n)$ обозначает сложностную функцию Шеннона в классе контактных схем без нулевых цепей, реализующих булевы функции от $n$ переменных.

ТЕОРема 1.17. При любом фиксированном $\varepsilon>0 u n \rightarrow \infty$

$$
L_{k}^{*}(n)>\frac{2^{n-1}}{\log _{2} \log _{2} n}(1-\varepsilon) .
$$


С. В. Здобнов в [45] усилил нижнюю оценку для $L_{k}^{*}(n)$ из теоремы 1.17 в два раза.

Сопоставление оценки для $L_{k}^{*}(n)$ из теоремы 1.17 с функцией Шеннона $L_{k}(n) \sim 2^{n} / n$ (см. теорему 1.4 ) в классе обычных контактных схем указывает на то, что отсутствие нулевых цепей в контактных схемах является существенным ограничением.

А. К. Пулатов и С.В. Здобнов исследовали сложность $\pi$-схем без нулевых цепей, реализующих булевы функции с заданным кодовым расстоянием. Такие функции определяются следующим образом.

Булева функция $f$ от $n$ переменных называется функцией с кодовым расстоянием $d, d \geqslant 2$, если расстояние Хемминга между любыми двумя наборами из $E^{n}$, на которых $f$ равна 1 , не меньше $d$.

А. К. Пулатов в [41] доказал следующее утверждение.

Теорема 1.18. Пусть булева функиия $f$ от $n$ переменных с кодовым расстоянием $d, d \geqslant 2$, равна 1 на s наборах из $E^{n}$. Тогда

$$
L_{\pi}^{*}(f) \geqslant d s^{d / n} .
$$

Аналогичные результаты были получены С. В. Здобновым в [44]. Им была доказана следующая теорема.

ТЕОрема 1.19. Пусть булева функиия $f$ от $n$ переменных с кодовым расстоянием $d$ равна 1 на $s$ наборах из $E^{n}$. Тогда при $d>n / 4 u s^{d / n} \geqslant 9 d$ справедливо неравенство

$$
L_{\pi}^{*}(f) \geqslant \frac{1}{4} s^{d / n} .
$$

И. О. Соколов в [48] показал, что двоичный логарифм числа контактов в минимальной контактной схеме без нулевых цепей, которая реализует характеристическую функцию кода Хемминга длины $n$, не превосходит

$$
\frac{1}{2} \log _{2}^{2}(n+1)+\frac{3}{2} \log _{2}(n+1)+1 .
$$

1.6. Контактные схемы ограниченной степени. Пусть $G$ - произвольный неориентированный граф с $r$ вершинами, две из которых являются полюсами. Обозначим через $\Delta_{i}, 1 \leqslant i \leqslant r$, число ребер в $G$, инцидентных $i$-й вершине. Величина $\Delta=\max _{1 \leqslant i \leqslant r} \Delta_{i}$ называется степенъю графа $G$. Под cmeneнъю контактной схемы, которая получена из $G$ путем замены ребер контактами, понимается степень графа $G$.

Обозначим через $L_{k}(f, \Delta)$ и $L_{\pi}(f, \Delta)$ минимальное число контактов, достаточное для реализации булевой функции $f$ контактной схемой и $\pi$-схемой степени не выше $\Delta$ соответственно.

Пусть $L_{k}(n, \Delta)=\max L_{k}(f, \Delta)$ и $L_{\pi}(n, \Delta)=\max L_{\pi}(f, \Delta)$, где максимум берется по всем булевым функциям от $n$ переменных.

В работе [49] доказаны следующие утверждения.

Теорема 1.20. При любом фиксированном $\Delta \geqslant 3 u n \rightarrow \infty$

$$
L_{k}(n, \Delta) \sim \frac{\Delta}{\Delta-2} \frac{2^{n}}{n}
$$


причем при любом фиксированном $\varepsilon>0$ среди булевых функиий от переменнъх $x_{1}, \ldots, x_{n}$ доля таких функиий $f$, что $L_{k}(f, \Delta)<(1-\varepsilon) \frac{\Delta}{\Delta-2} \frac{2^{n}}{n}$, стремится к нулю с ростом $n$.

ТЕОрема 1.21. При любом фиксированном $\Delta \geqslant 3 u n \rightarrow \infty$

$$
L_{\pi}(n, \Delta) \sim \frac{2^{n}}{\log _{2} n},
$$

причем при любом фиксированном $\varepsilon>0$ среди булевых функиий от переменнъх $x_{1}, \ldots, x_{n}$ доля таких функиий $f$, что $L_{\pi}(f, \Delta)<(1-\varepsilon) \frac{2^{n}}{\log _{2} n}$, стремится $\kappa$ нулю с ростом $n$.

Ясно, что в классе контактных схем степени не более двух можно реализовать весьма мало булевых функций. Поэтому ограничение $\Delta \geqslant 3$ является необходимым.

1.7. Метод Храпченко для $\pi$-схем. Пусть $f$ - произвольная (быть может, не всюду определенная) булева функция от $n$ переменных, $M_{0}(f)$ - множество вершин из $E^{n}$, на которых $f$ равна $0, M_{1}(f)$ - множество вершин из $E^{n}$, на которых $f$ равна $1, R(f)$ - множество ребер в $E^{n}$, каждое из которых соединяет некоторую вершину из $M_{0}(f)$ с какой-либо вершиной из $M_{1}(f)$.

В работе [50] В. М. Храпченко предложил метод получения нижней оценки для сложности $\pi$-схем, реализующих произвольные булевы функции. Эта оценка зависит от параметров $M_{0}(f), M_{1}(f)$ и $R(f)$ рассматриваемой функции $f$. Им доказана следующая теорема.

ТЕОРема 1.22. Для произвольной булевой функиии $f$ от $n$ переменных справедливо неравенство

$$
L_{\pi}(f) \geqslant \frac{|R(f)|^{2}}{\left|M_{0}(f)\right| \cdot\left|M_{1}(f)\right|} .
$$

Легко видеть, что для любой булевой функции $f$ от $n$ переменных справедливо соотношение

$$
\frac{|R(f)|^{2}}{\left|M_{0}(f)\right| \cdot\left|M_{1}(f)\right|} \leqslant \frac{\left(n \min \left\{\left|M_{0}(f)\right|,\left|M_{1}(f)\right|\right\}\right)^{2}}{\left|M_{0}(f)\right| \cdot\left|M_{1}(f)\right|} \leqslant n^{2} .
$$

Следовательно, метод Храпченко позволяет получать нижние оценки сложности, не превосходящие величины $n^{2}$. Оценка $n^{2}$ достижима, поскольку для линейной функции $f_{0}\left(x_{1}, \ldots, x_{n}\right)=x_{1}+\cdots+x_{n}(\bmod 2)$ имеем $\left|M_{0}\left(f_{0}\right)\right|=$ $\left|M_{1}\left(f_{0}\right)\right|=2^{n-1}$ и $\left|R\left(f_{0}\right)\right|=n \cdot 2^{n-1}$. Поэтому для функции $f_{0}$ справедливо неравенство $L_{\pi}\left(f_{0}\right) \geqslant n^{2}$. Вместе с тем С. В. Яблонский в [51] описал метод синтеза такой $\pi$-схемы, реализующей функцию $f_{0}\left(x_{1}, \ldots, x_{n}\right)$, в которой содержится не более $\frac{9}{8} n^{2}$ контактов. Более того, если $n$ равно степени двойки, то в этой схеме содержится $n^{2}$ контактов. Отсюда и из теоремы 1.22 следует, что минимальная $\pi$-схема, реализующая функцию $f_{0}\left(x_{1}, \ldots, x_{n}\right)=x_{1}+\cdots+x_{n}(\bmod 2)$, при $n=2^{s}$ содержит $n^{2}$ контактов. В остальных случаях $L_{\pi}\left(f_{0}\right)=\Omega\left(n^{2}\right)$. 
Развитие метода Храпченко имеется в работе К.Л. Рычкова [52]. В ней задача о нижних оценках сложности $\pi$-схем, реализующих булевы функции от $n$ переменных, сведена к задаче о нижних оценках числа более специальных подмножеств в $E^{n}$, нежели в работах В. М. Храпченко.

1.8. Симметрические булевы функции. Одним из простейших классов булевых функций является класс симметрических функций.

Булева функция называется симметрической, если ее значения не изменяются при любой перестановке ее переменных. Примерами симметрических булевых функций являются:

1) $x_{1} x_{2} \cdots x_{n}-$ конъюнкция переменных;

2) $\bar{x}_{1} \vee \bar{x}_{2} \vee \cdots \vee \bar{x}_{n}-$ дизъюнкция отрицаний переменных;

3) $x_{1}+\cdots+x_{n}(\bmod 2)-$ линейная функция.

Из определения следует, что симметрическая булева функция $f\left(x_{1}, \ldots, x_{n}\right)$, равная единице на двоичном наборе $\widetilde{\sigma}=\left(\sigma_{1}, \ldots, \sigma_{n}\right)$, равна единице и на любом другом двоичном наборе той же длины, имеющем столько же единиц, сколько содержится в $\widetilde{\sigma}$.

Если симметрическая булева функция $f$ от $n$ переменных равна единице на двоичных наборах длины $n$ с $a$ единицами, то число $a$ называется рабочим числом функции $f$. Очевидно, любая симметрическая булева функция полностью определяется набором своих рабочих чисел.

Симметрическая булева функция с одним рабочим числом называется элементарной симметрической булевой функцией. К. Шеннон в [20] показал, что любая элементарная симметрическая булева функция от $n$ переменных может быть реализована двухполюсной контактной схемой, содержащей не более $\left\lfloor\frac{1}{2} n^{2}(1+o(1))\right\rfloor$ контактов.

Позднее этот результат усилен О.Б. Лупановым. В работе [53] им была доказана следующая теорема.

ТЕОРема 1.23. Число контактов в минимальной контактной схеме, реализующей произвольную элементарную симметрическую булеву функцию от $n$ переменных, не превосходит величины

$$
\frac{1}{\log _{2} e} \frac{n \log _{2}^{2} n}{\log _{2} \log _{2} n},
$$

где $\varepsilon_{n} \rightarrow 0$ при $n \rightarrow \infty$.

Для $\pi$-схем О. Б. Лупанов в [53] установил следующий факт.

ТЕОРема 1.24. Число контактов в минимальной $\pi$-схеме, реализующей произвольную элементарную симметрическую булеву функиию от $n$ переменных, не превосходит величинь

$$
n^{\left(1+\varepsilon_{n}\right) \log _{2} \log _{2} n}
$$

где $\varepsilon_{n} \rightarrow 0$ при $n \rightarrow \infty$. 
Обозначим через $L_{k}^{s}(n)$ минимальное число контактов, достаточное для построения контактной схемы, реализующей произвольную симметрическую булеву функцию от $n$ переменных.

Сначала К. Шеннон в [19] доказал, что $L_{k}^{s}(n) \leqslant n^{2}$. Затем в [20] он показал, что $L_{k}^{s}(n)<n^{2}-n+2$. Более низкая верхняя оценка для $L_{k}^{s}(n)$ была получена Г. Н. Поваровым и С. В. Яблонским (см. [21]):

$$
L_{k}^{s}(n)=n^{2}-2 n \log _{2} n+O(n) .
$$

Позднее О. Б. Лупанов существенно понизил эту оценку. Использовав оригинальный прием "разложения симметрических булевых функций по элементарным симметрическим булевым функциям", в работе [53] он описал такой метод построения контактных схем, реализующих симметрические булевы функции, из которого вытекает следующее утверждение.

TEOPEMA 1.25. При $n \rightarrow \infty u \varepsilon_{n} \rightarrow 0$

$$
L_{k}^{s}(n) \leqslant \frac{2 n^{2}}{\log _{2} n}\left(1+\varepsilon_{n}\right) .
$$

Сложность реализации симметрических булевых функций в классе $\pi$-схем изучали В.К. Коробков и О. Б. Лупанов. В. К. Коробков описал такой метод построения $\pi$-схем, реализующих симметрические булевы функции от $n$ переменных, с помощью которого удается получать $\pi$-схемы, в каждой из которых содержится не более $n^{\left(\frac{1}{2}+\varepsilon_{n}\right) \log _{2} n}$ контактов, где $\varepsilon_{n} \rightarrow 0$ при $n \rightarrow \infty$.

Этот результат был существенно улучшен О.Б. Лупановым. После теоремы 1.24 в [53] им доказана следующая теорема.

Теорема 1.26. Число контактов в минимальной $\pi$-схеме, реализующей произвольную симметрическую булеву функцию от $n$ переменных, не превосходит величины $n^{\left(1+\varepsilon_{n}\right) \log _{2} \log _{2} n}$, где $\varepsilon_{n} \rightarrow 0$ при $n \rightarrow \infty$.

1.9. Инвариантные классы булевых функций. Понятие инвариантного класса булевых функций ввел С. В. Яблонский (см., например, [5]).

Множество $Q$ булевых функций называется инвариантным классом, если $Q$ замкнуто относительно следующих операций:

1) добавления и изъятия несущественных переменных;

2) переименования переменных (без отождествления);

3) подстановки констант 0 и 1 вместо некоторых переменных.

Очевидными примерами инвариантных классов являются:

(a) множество всех булевых функций;

(b) множество булевых функций, существенно зависящих не более чем от $n$ переменных;

(с) множество монотонных булевых функций.

С каждым инвариантным классом $Q$ булевых функций связывается числовая функция $P_{Q}(n)$ - число функций $f\left(x_{1}, \ldots, x_{n}\right)$ от $n$ переменных из класса $Q$. Очевидно, что $P_{Q}(n) \leqslant 2^{2^{n}}$. С. В. Яблонский заметил, что последовательность $\sqrt[2^{n}]{P_{Q}(n)}$ не возрастает по $n$ и поэтому имеет предел. Число 
$\sigma_{Q}=\log _{2}\left(\lim _{n \rightarrow \infty} \sqrt[2^{n}]{P_{Q}(n)}\right)$ называется параметром инвариантного класса $Q$. Очевидно, что для любого инвариантного класса $Q$ с параметром $\sigma_{Q}$ справедливо соотношение $0 \leqslant \sigma_{Q} \leqslant 1$. Равенство $\sigma_{Q}=1$ имеет место тогда и только тогда, когда $Q$ совпадает с множеством всех булевых функций. Инвариантный класс $Q$ с $\sigma_{Q}=0$ называется нулевым. Для всякого $\sigma, 0 \leqslant \sigma<1$, существует континуальное семейство попарно различных инвариантных классов $Q$ с параметром $\sigma_{Q}=\sigma$.

Пусть $L_{Q}(f)$ обозначает число контактов в минимальной контактной схеме, реализующей функцию $f$ из $Q$, и пусть $L_{Q}(n)=\max L_{Q}(f)$, где максимум берется по всем функциям из $Q$ от переменных $x_{1}, \ldots, x_{n}$.

С. В. Яблонский в [5] установил следующие факты.

Теорема 1.27. Для любого инвариантного класса $Q$ булевых функиий с параметром $\sigma_{Q}, 0<\sigma_{Q} \leqslant 1$, при $n \rightarrow \infty$ справедливо соотношение

$$
L_{Q}(n) \sim \sigma \frac{2^{n}}{n}
$$

ТеОРема 1.28. Для любого инвариантного класса $Q$ булевых функиий с параметром $\sigma_{Q}=0$ при $n \rightarrow \infty$ имеет место соотношение

$$
\lim _{n \rightarrow \infty} \frac{L_{Q}(n)}{L_{k}(n)}=0 .
$$

Теорема 1.28 утверждает, что булевы функции из класса $Q$ с параметром $\sigma_{Q}=0$ допускают более простую реализацию, чем в общем случае.

\subsection{0. Нижние оценки сложности минимальных контактных схем.} Проблема нижних оценок сложности, имеющая более полувековую историю, одна из наиболее трудных в дискретной математике. Она представляет большой интерес как для теории, так и для практики.

В прикладном плане решение проблемы нижних оценок сложности способствовало бы разработке более эффективных алгоритмов и созданию методик по оценке качества алгоритмов и программ.

Проблема нижних оценок сложности схем состоит в том, чтобы установить "хорошие" нижние оценки сложности вычислений конкретных булевых функций.

Несмотря на значительные усилия многих математиков на протяжении нескольких десятилетий, в решении этой проблемы нет заметного прогресса. В случае произвольных булевых схем известны лишь линейные (относительно числа переменных вычисляемых функций) нижние оценки сложности минимальных схем (см. п. 2.14). В классе всех контактных схем известны невысокие нелинейные нижние оценки.

Общее число известных нижних оценок сложности контактных схем невелико. Перечислим основные из них, начав с таких контактных схем, для которых доказана минимальность. 
1) К. Кардо в [54] доказал минимальность известной контактной схемы, реализующей линейную булеву функцию $f\left(x_{1}, \ldots, x_{n}\right)=x_{1}+\cdots+x_{n}(\bmod 2)($ см. рис. 1.4). Эта схема содержит $4 n-4$ контакта.
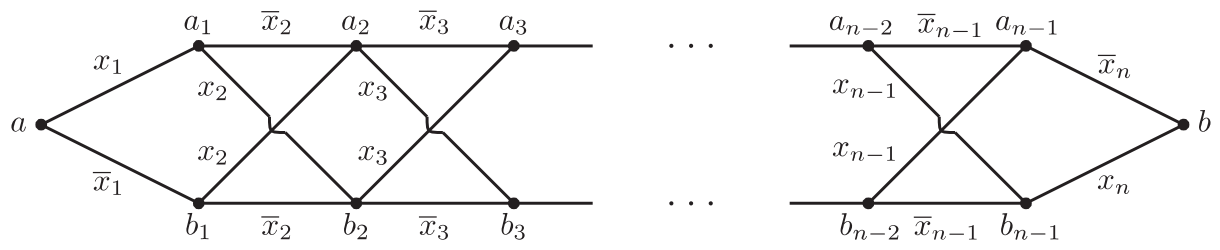

Рис. 1.4

Другое доказательство этого факта привел С. М. Вартанян [55]. Впоследствии предложенный К. Кардо метод доказательства минимальности контактной схемы, изображенной на рис. 1.4, применял Н. П. Редькин для получения точного значения $4 n-4$ для сложности булевых схем, реализующих линейные булевы функции (см. п. 2.14).

2) 3. Е. Королева [56] доказала, что в минимальной контактной схеме, которая вычисляет функцию прибавления единицы к младшему разряду $n$-разрядного числа, заданного в позиционной двоичной системе счисления, содержится $5 n-6$ контактов.

3) Э. Мур в [24] доказал, что число контактов в минимальном разделительном $\left(1,2^{m}\right)$-полюснике, реализующем все элементарные конъюнкции от переменных $x_{1}, \ldots, x_{m}$, равно $2^{m+1}-2$ (подробнее см. в п. 1.2).

4) А. Д. Коршунов в [25] установил, что число контактов в минимальном $(1, k)$-полюснике из переключательных контактов, реализующем систему $k$ попарно ортогональных булевых функций, не меньше $2 k-2$ (определение контактной схемы из переключательных контактов имеется в п. 1.2). Следовательно, если система состоит из $2^{m}$ элементарных конъюнкций от переменных $x_{1}, \ldots, x_{m}$, то в минимальном $\left(1,2^{m}\right)$-полюснике из переключательных контактов, реализующем эту систему, содержится $2^{m+1}-2$ контактов.

Теперь перечислим результаты по нелинейным нижним оценкам сложности контактных схем.

5) Первую нелинейную нижнюю оценку сложности контактных схем установил Э. И. Нечипорук. В работе [57] им указана последовательность конкретных булевых функций $f_{1}\left(x_{1}\right), f_{2}\left(x_{1}, x_{2}\right), \ldots, f_{n}\left(x_{1}, \ldots, x_{n}\right), \ldots$ такая, что минимальная контактная схема, вычисляющая функцию $f_{n}\left(x_{1}, \ldots, x_{n}\right)$, содержит не менее $\left(n / \log _{2} n\right)^{2}$ контактов.

В этой же работе была установлена аналогичная нижняя оценка сложности минимальных формул (подробнее об этом будет сказано в п. 3.5).

Эти оценки были самыми высокими из известных в то время для указанных классов схем. Для успешного применения метода Нечипорука необходимо, чтобы у рассматриваемой булевой функции было много подфункций.

6) Э. Шпекер [58] предложил метод получения нелинейных нижних оценок сложности реализации конкретных булевых функций $\pi$-схемами и булевыми схемами без ветвлений в базисах $\{\&,-\},\left\{\&, \oplus,^{-}\right\}$. Это второй после метода 
Нечипорука [57] метод получения таких оценок, причем если метод Нечипорука более эффективен по отношению к "сильно несимметрическим функциям", то метод Шпекера более эффективен по отношению к функциям, обладающим той или иной симметрией. Правда, в отличие от метода Нечипорука метод Шпекера позволяет получать лишь весьма слабую нелинейность. Она пропорциональна количеству логарифмирований (по основанию 2 ) числа $n(n-$ число переменных функции), после которого впервые получается число, не превосходящее 1. Поэтому метод Шпекера интересен прежде всего с принципиальной точки зрения.

7) В. М. Храпченко в [50] доказал, что в любой $\pi$-схеме, реализующей линейную булеву функцию от $n$ переменных, содержится не менее $n^{2}$ контактов.

8) А. Е. Андреев [59] на основе обобщения метода Субботовской для формул с использованием универсальной булевой функции Нечипорука из [57] построил такую булеву функцию от $n$ переменных, $n=2,3, \ldots$, что в каждой $\pi$-схеме, реализующей эту функцию, содержится по порядку не менее $\frac{n^{5 / 2}}{\left(\log _{2} n\right)^{(3 / 2) \log _{2} \log _{2} n}}$ контактов.

9) М.И. Гринчук в [60] нашел эффективный критерий реализуемости последовательностей симметрических булевых функций контактными схемами со сложностью $O(n)$ (этот критерий назовем критерием линейности). Эффективность критерия линейности состоит в том, что он позволяет по функциональным характеристикам последовательности функций судить о ее сложностных характеристиках. Позднее в [61] М. И. Гринчук показал, что сложность любой контактной схемы, которая реализует симметрическую булеву функцию от $n$ переменных, не удовлетворяющую критерию линейности, не менее $n \varphi(n)$, где $\varphi(n) \rightarrow \infty$ при $n \rightarrow \infty$ очень медленно.

1.11. Дизъюнктивные нормальные формы. Дизъюнктивные нормальные формы (д.н.ф.) широко используются при реализации булевых функций. Каждая д.н.ф. (тождественно не равная нулю) может быть реализована двухполюсной контактной $\pi$-схемой, состоящей из таких параллельных простых цепей, которые пересекаются только в полюсах схемы. Поэтому све́дения о д.н.ф. излагаются в разделе о контактных схемах, а не в разделе о булевых схемах без ветвлений.

Дизъюнктивные нормальные формы определяются следующим образом.

Дизгюнктивной нормальной формой (д.н.ф.) называется дизъюнкция различных элементарных конъюнкций. Известно, что каждая булева функция, отличная от 0 , может быть задана дизъюнктивной нормальной формой и не единственной.

Исследованию дизъюнктивных нормальных форм посвящены обзорные статьи Ю.Л. Васильева и В.В. Глаголева [13], Ю. И. Журавлёва [62]-[64], [15], А. А. Сапоженко и И. П. Чухрова [17] и отдельные главы в книгах [3], [6], [8], [9]. Среди дизъюнктивных нормальных форм наиболее известными являются следующие: совершенные д.н.ф., сокращенные д.н.ф., тупиковые д.н.ф., кратчайшие д.н.ф. и минимальные д.н.ф.

Д.н.ф. $D$, задающая булеву функцию от $n$ переменных, называется совершенной, если $D$ состоит из элементарных конъюнкций длины $n$. 
Д.н.ф. $D$, задающая булеву функцию $f$, называется сокращенной, если $D$ состоит из всех таких простых конъюнкций, что после удаления хотя бы одного литерала из некоторой конъюнкции получается д.н.ф., которая задает функцию, отличную от $f$.

Д.н.ф. $D$, задающая булеву функцию $f$, называется тупиковой, если $D$ состоит из всех таких простых конъюнкций, что после удаления хотя бы одного литерала из некоторой конъюнкции или удаления какой-либо конъюнкции из $D$ получается д.н.ф., которая задает функцию, отличную от $f$.

Д.н.ф. $D$, задающая булеву функцию $f$, называется кратчайшей, если в $D$ содержится наименьшее число конъюнкций среди всех д.н.ф., задающих булеву функцию $f$.

Д.н.ф. $D$, задающая булеву функцию $f$, называется минимальной, если в $D$ содержится наименьшее число литералов среди всех д.н.ф., задающих булеву функцию $f$.

Легко видеть, что тупиковая, кратчайшая и минимальная д.н.ф., задающие булеву функцию $f$, получаются из сокращенной д.н.ф. булевой функции удалением подходящих конъюнкций.

Название "сокращенная д.н.ф." является неудачным. Действительно, пусть $f_{0}$ - булева функция от $n$ переменных, равная 1 только на таких двоичных наборах длины $n$, в каждом из которых содержится не менее $k=\lfloor n / 3\rfloor$ и не более $2 k$ единиц. Тогда сокращенная д.н.ф. функции $f_{0}$ состоит из $\left(\begin{array}{l}n \\ k\end{array}\right)\left(\begin{array}{c}n-k \\ k\end{array}\right)$ конъюнкций, что существенно больше $2^{n}$ при $n \rightarrow \infty$.

Наиболее востребованными являются кратчайшие и минимальные д.н.ф. О них будет идти речь ниже.

Некоторое время полагали, что минимальные д.н.ф. любой булевой функции находятся среди ее кратчайших д.н.ф. Это предположение оказалось ошибочным. Его опроверг Ю. И. Журавлёв, построив в [65] пример булевой функции от 11 переменных, у которой среди всех кратчайших д.н.ф. нет ни одной минимальной д.н.ф.

Дальнейшее изучение этого явления было проведено в работе [66]. Для формулировки основных результатов этой работы введем следующие обозначения.

Пусть $f$ - произвольная булева функция и

$L_{1}(f)$ - число литералов в кратчайшей д.н.ф., задающей функцию $f$, с минимальным числом литералов;

$L_{2}(f)$ - число литералов в минимальной д.н.ф., задающей функцию $f$;

$\tau(f)=L_{1}(f) / L_{2}(f)$;

$\tau(n)=\max \tau(f)$, где максимум берется по всем булевым функциям $f$ от $n$ переменных.

Основным результатом работы [66] является следующая теорема.

TEOPEMA 1.29. При $n \rightarrow \infty$

$$
\tau(n) \sim n / 2
$$

Исследованию сложности кратчайших и минимальных д.н.ф., а также других проблем дизъюнктивных нормальных форм булевых функций посвящены 
работы Ю.И. Журавлёва [62], [15] и другие. В своих работах Ю.И. Журавлёв ввел понятие локального алгоритма и разработал теорию локальных алгоритмов. В частности, им были доказаны теорема единственности и теорема существования наилучшего локального алгоритма.

На языке локальных алгоритмов естественно формулируются и решаются некоторые задачи упрощения дизъюнктивных нормальных форм булевых функций и некоторые задачи по обработке информации.

Перечислим результаты, относящиеся к оценке сложности кратчайших и минимальных д.н.ф. булевых функций.

Пусть $f\left(x_{1}, \ldots, x_{n}\right)$ - произвольная булева функция от $n$ переменных, $S(f)$ число конъюнкций в кратчайшей д.н.ф. функции $f$ и

$$
S(n)=\max S\left(f\left(x_{1}, \ldots, x_{n}\right)\right),
$$

где максимум берется по всем булевым функциям $f$ от $n$ переменных.

Известно, что $S(n)=2^{n-1}$. Это значение достигается на следующих линейных функциях:

$$
\begin{aligned}
& f_{1}\left(x_{1}, \ldots, x_{n}\right)=x_{1}+\cdots+x_{n} \quad(\bmod 2), \\
& f_{2}\left(x_{1}, \ldots, x_{n}\right)=x_{1}+\cdots+x_{n}+1 \quad(\bmod 2) .
\end{aligned}
$$

Вместе с тем в любой кратчайшей д.н.ф. почти каждой булевой функции от переменных $x_{1}, \ldots, x_{n}$ содержится не менее $2^{n-3} / n$ конъюнкций. Впервые этот факт обнаружил Ю. И. Журавлёв. В работе [63; лемма на с. 26], им показано, что в любой кратчайшей (минимальной) д.н.ф. почти каждой булевой функции от $n$ переменных отсутствуют конъюнкции длины менее $n-1-\log _{2} n$. Пользуясь этим фактом и тем, что при $n \rightarrow \infty$ почти каждая булева функция от $n$ переменных равна 1 на $2^{n-1}(1+o(1))$ наборах из $E^{n}$, получаем, что в любой кратчайшей д.н.ф. такой функции содержится не менее $2^{n-2-\log _{2} n}(1+o(1))>$ $2^{(n-3) / n}$ конъюнкций.

Позднее В. В. Глаголев в [67] показал, что для почти каждой булевой функции $f\left(x_{1}, \ldots, x_{n}\right)$ справедливо неравенство

$$
S\left(f\left(x_{1}, \ldots, x_{n}\right)\right)>\frac{1-\varepsilon_{n}}{\log _{2} n \cdot \log _{2} \log _{2} n} \cdot 2^{n-1},
$$

где $\varepsilon_{n} \rightarrow 0$ при $n \rightarrow \infty$.

Оказалось, что случаи, когда $S\left(f\left(x_{1}, \ldots, x_{n}\right)\right)$ близка к $2^{n-1}$, являются исключительными. Этот факт установил В. В. Глаголев. В [68] он показал, что в кратчайшей д.н.ф. почти каждой булевой функции от $n$ переменных число конъюнкций не превосходит $\frac{\log _{2} \log _{2} n}{\log _{2} n} \cdot 2^{n}$. В 1969 г. автор настоящего обзора в [69] усилил этот результат до следующего: в кратчайшей д.н.ф. почти каждой булевой функции от $n$ переменных содержится не более $2^{n+2} / \log _{2} n$ конъюнкций.

Аналогичный результат получил А. А. Сапоженко. В [70], используя жадный алгоритм для покрытия множества специальными подмножествами, он показал, что в кратчайшей д.н.ф. почти каждой булевой функции от $n$ переменных содержится не более $2^{n} / \ln n$ конъюнкций. 
Наконец, в [71], [72] автором был предложен оригинальный метод построения д.н.ф. произвольной булевой функции, из которого извлекается следующее утверждение: в кратчайшей д.н.ф. почти каждой булевой функции от $n$ переменных содержится не более $2.63 \cdot 2^{n} /\left(\log _{2} n \cdot \log _{2} \log _{2} n\right)$ конъюнкций. Отсюда и из [67] вытекает следующая теорема.

ТЕОРема 1.30. Число конбюнкиий в кратчайшей д.н.ф. почти каждой булевой функиии от $n$ переменных по порядку равно $2^{n} /\left(\log _{2} n \cdot \log _{2} \log _{2} n\right)$.

Вместе с тем Р. Г. Нигматуллин с использованием введенного им вариационного принципа [73] доказал, что для почти всех булевых функций $f\left(x_{1}, \ldots, x_{n}\right)$ величины $S\left(f\left(x_{1}, \ldots, x_{n}\right)\right)$ асимптотически совпадают. Однако вид этой асимптотики до сих пор остается неизвестным.

ЗАМЕчАНИЕ 1. После выхода в свет работы [71] появилась публикация А. Е. Андреева [74], в которой утверждается, что

$$
S\left(f\left(x_{1}, \ldots, x_{n}\right)\right)<\frac{1.2 \cdot 2^{n}}{\log _{2} n \cdot \log _{2} \log _{2} n}
$$

для почти каждой булевой функции $f\left(x_{1}, \ldots, x_{n}\right)$ от $n$ переменных. Однако приведенное в [74] доказательство является неполным, поскольку в нем используется независимость и одинаковая распределенность некоторых случайных величин, что не доказывается в статье.

ЗАмЕчАниЕ 2. В работе [75] Н. Пиппенджера имеется следующее утверждение: в кратчайшей д.н.ф. почти каждой булевой функции от $n$ переменных содержится не более $1.54169 \cdot 2^{n} /\left(\log _{2} n \cdot \log _{2} \log _{2} n\right)$ конъюнкций.

Для произвольной булевой функции $f$ обозначим через $m(f)$ число литералов в минимальной д.н.ф. функции $f$. Если $f$ является функцией от $n$ переменных, то очевидно, что $m(f)<n S(f)$. Отсюда и из верхней оценки для $S(f)$ из теоремы 1.30 следует, что в минимальной д.н.ф. почти каждой булевой функции от $n$ переменных содержится не более $2.63 n \cdot 2^{n} /\left(\log _{2} n \cdot \log _{2} \log _{2} n\right)$ литералов. Вместе с тем, поскольку в минимальной д.н.ф. почти каждой булевой функции от $n$ переменных отсутствуют конъюнкции длины менее $n-\log _{2} n$, из [67] следует, что в любой минимальной д.н.ф. почти каждой булевой функции от $n$ переменных по порядку содержится не менее $n \cdot 2^{n}\left(1-\varepsilon_{n}\right) /\left(\log _{2} n\right.$. $\left.\log _{2} \log _{2} n\right)$ литералов. Из сказанного вытекает следующее утверждение.

Теорема 1.31. Число литералов в минимальной д.н.ф. почти каждой булевой функции от $n$ переменных по порядку равно $n \cdot 2^{n} /\left(\log _{2} n \cdot \log _{2} \log _{2} n\right)$.

1.12. Разное. Известно, что для реализации булевых функций использование, наряду с контактами, вентилей, сопротивлений и других элементарных средств иногда позволяет существенно упростить схемы. Может показаться, что расширение используемых средств дает возможность значительно упростить схемы, реализующие произвольные булевы функции.

Убедимся, что это не так для релейно-контактных и контактно-вентильных схем. 
1. Релейно-контактные схемы. Релейно-контактная схема отличается от контактной схемы тем, что в ней кроме контактов исходных переменных имеются также обмотки реле и контакты промежуточных реле. Реализация булевых функций релейно-контактными схемами возможна несколькими способами. Ниже рассматривается реализация, которая описана О.Б. Лупановым в $[76]$.

Одной из целей работы [76] является выяснение асимптотического поведения функции Шеннона о сложности релейно-контактных схем и описание методов синтеза таких схем, которые позволяют строить асимптотически наилучшие схемы, реализующие почти все булевы функции.

Для формулировки основного результата о сложности релейно-контактных схем введем следующие понятия.

Пусть $S$ - произвольная двухполюсная релейно-контактная схема; $m_{1}(S)-$ число обмоток в схеме $S ; m_{2}(S)$ - число контактов исходных переменных в схеме $S ; m_{3}(S)$ - число контактов промежуточных реле в схеме $S$.

Пусть $\alpha, \beta, \gamma$ - неотрицательные числа (стоимость обмотки реле, контакта исходной переменной и контакта промежуточного реле соответственно). Число $L_{r, k}=\alpha m_{1}(S)+\beta m_{2}(S)+\gamma m_{3}(S)$ называется сложностью схемы $S$.

Как и выше, определяются сложностные функции Шеннона $L_{r, k}(f)$ и $L_{r, k}(n)$. Основной результат работы [76] о сложности релейно-контактных схем состоит в нахождении асимптотики для функции $L_{r, k}(n)$. Сначала в них О.Б. Лупанов с использованием мощностных соображений нашел достаточно точные нижние оценки для функции $L_{r, k}(n)$, а затем описал такие методы синтеза релейно-контактных схем, которые позволяют получать схемы, сложность которых асимптотически совпадает с нижними оценками.

Поведение функции $L_{r, k}(n)$ существенно зависит от соотношений параметров $\alpha, \beta$ и $\gamma$. Вместе с тем, функция $L_{r, k}(n)$ с точностью до порядка равна $2^{n} / n$.

2. Вентильные схемы. Вентильные схемы непосредственно не связаны с контактными схемами, а используются при реализации бинарных матриц. Тем не менее некоторые результаты из теории вентильных схем иногда существенным образом используются при синтезе контактных схем. Поэтому вкратце осветим такие результаты.

Вентильные схемы определяются следующим образом. Ориентированный граф $S$ называется вентильной $(p, q)$-схемой, если:

1) в $S$ выделено $p$ вершин - входных полюсов (входов) и $q$ других вершин выходных полюсов (выходов);

2) в $S$ нет ориентированных цепей (путей) от одного входа к другому входу, от одного выхода к другому выходу и от выходов к входам.

Дуги такого графа называются вентилями. Число вентилей, содержащихся в самой длинной простой цепи, ориентированной от входа к выходу в $S$, называется глубиной вентильной схемы $S$.

С каждой вентильной $(p, q)$-схемой связывается матрица размера $p \times q$ из нулей и единиц (бинарная матрица) - матрица проводимостей $A=\left(a_{i j}\right)\left(a_{i j}=1\right.$ тогда и только тогда, когда в $S$ имеется хотя бы один путь от полюса $i$ к полюсу $j$ ). В этом случае говорят, что вентильная $(p, q)$-схема $S$ реализует матрицу $A$. 
Одной из основных задач теории вентильных схем является задача построения по заданной бинарной матрице $A$ вентильной схемы с минимально возможным числом вентилей, которая реализует матрицу $A$.

Для характеристики сложности вентильных схем вводится величина $B\left(p_{n}, q_{n}\right)$ $\left(B_{r}\left(p_{n}, q_{n}\right)\right)$ - минимальное число вентилей, достаточное для построения вентильной $\left(p_{n}, q_{n}\right)$-схемы (вентильной $\left(p_{n}, q_{n}\right)$-схемы глубины $\left.r\right)$, реализующей бинарную матрицу размера $p_{n} \times q_{n}$.

Первые результаты об оценке величин $B\left(p_{n}, q_{n}\right)$ и $B_{r}\left(p_{n}, q_{n}\right)$ получил О. Б. Лупанов. В работе [77] им доказана следующая теорема.

Теорема 1.32. Пусть при $n \rightarrow \infty$ последовательность $\left(p_{1}, q_{1}\right),\left(p_{2}, q_{2}\right), \ldots$ удовлетворяет условиям $p_{n} \leqslant q_{n}, p_{n} \rightarrow \infty u \log _{2} q_{n}=o\left(p_{n}\right)$. Тогда

$$
B_{r}\left(p_{n}, q_{n}\right) \sim \frac{p_{n} q_{n}}{\log _{2} q_{n}}
$$

Кроме того, если $\log _{2} p_{n}=o\left(\log _{2} q_{n}\right)$, mо

$$
B\left(p_{n}, q_{n}\right) \sim \frac{p_{n} q_{n}}{\log _{2} q_{n}} .
$$

Наряду с задачей о сложности реализации произвольных бинарных матриц (заданных размеров) вентильными схемами рассматривались также задачи о сложности реализации специальных бинарных матриц и конкретных матриц. Приведем два примера.

Пусть $B_{r}\left(p_{n}, q_{n}, \alpha\right)$ обозначает минимальное число вентилей, достаточное для построения вентильной $\left(p_{n}, q_{n}\right)$-схемы глубины $r$, реализующей произвольную бинарную матрицу размера $p_{n} \times q_{n}$ с $\alpha p_{n} q_{n}$ единицами и $(1-\alpha) p_{n} q_{n}$ нулями, $0 \leqslant \alpha \leqslant 1$. Требуется найти асимптотику величины $B_{r}\left(p_{n}, q_{n}, \alpha\right)$ при различных фиксированных $r, \alpha$ и $p_{n}+q_{n} \rightarrow \infty$ при $n \rightarrow \infty$.

При некоторых $\alpha$ и соотношениях между параметрами $p_{n}, q_{n}$ такую асимптотику нашел Э. И. Нечипорук в [78]. Она имеет следующий вид.

Пусть $B_{r}^{\gamma}\left(p_{n}, q_{n}\right)$ обозначает минимальное число вентилей, достаточное для построения вентильной $\left(p_{n}, q_{n}\right)$-схемы глубины $r$, реализующей произвольную бинарную матрицу размера $p_{n} \times q_{n}$, в которой число определенных элементов нулей и единиц - равно $\left\lfloor\gamma p_{n} q_{n}\right\rfloor$, а остальные элементы не определены; при реализации такой матрицы вентильной $\left(p_{n}, q_{n}\right)$-схемой они заменяются на нули и единицы так, чтобы реализация была простейшей (например, число вентилей оказалось минимально возможным), $0<\gamma<1$.

Э. И. Нечипоруком [78] доказана следующая теорема.

TEOPEMA 1.33. Пусть $p_{n} \leqslant q_{n}, p_{n} \rightarrow \infty u \frac{\log _{2} q_{n}}{-\log _{2} \gamma} \rightarrow \infty$ nрu $n \rightarrow \infty$. Тогда

$$
B_{r}^{\gamma}\left(p_{n}, q_{n}\right) \sim \gamma \frac{p_{n} q_{n}}{\log _{2} q_{n}} .
$$


3. Контактно-вентильные схемы. Двухполосной контактно-вентильной схемой называется сеть без петель с двумя выделенными вершинами (полюсами), состоящими из вершин, замыкающих и размыкающих контактов, а также вентилей. Один полюс такой схемы называется входным, а второй - выходным.

Простая цепь, ведущая от входного полюса $a$ к выходному $b$ в контактно-вентильной схеме $S$, замкнута, если замкнуты все ее контакты и каждый вентиль, содержащийся в этой цепи, ориентирован от полюса $a$ к полюсу $b$.

Проводимостью от полюса $a$ к полюсу $b$ называется совокупность всех замкнутых простых цепей от $a$ к $b$. Таким образом, проводимость от полюса $a$ к полюсу $b$ в схеме $S$ задается булевой функцией от тех переменных, которые содержатся в $S$.

Обозначим через $L_{k b}(n)$ минимальное натуральное число такое, что любую булеву функцию от $n$ переменных можно реализовать, как функцию проводимости, контактно-вентильной схемой, в которой общее число вентилей и контактов не превосходит $L_{k b}(n)$.

О. Б. Лупанов в [77] доказал следующее утверждение.

TEOPEMA 1.34. При $n \rightarrow \infty$

$$
L_{k b}(n) \sim \frac{2^{n}}{n}
$$

Обозначим через $\widetilde{L}_{k b}(n)$ минимальное натуральное число такое, что любую булеву функцию от $n$ переменных можно реализовать как функцию проводимости некоторой контактно-вентильной схемой, содержащей не более $\widetilde{L}_{k b}(n)$ контактов. Для величины $\widetilde{L}_{k b}(n)$ получены следующие оценки.

О. Б. Лупанов в [77] доказал, что при любом фиксированном $\varepsilon>0$ и $n \rightarrow \infty$ справедливы неравенства

$$
\frac{1}{2} \cdot 2^{n / 2}(1-\varepsilon)<\widetilde{L}_{k b}(n)< \begin{cases}2^{n / 2+2} & \text { при четном } n \\ \frac{3}{\sqrt{2}} \cdot 2^{n / 2} & \text { при нечетном } n .\end{cases}
$$

Эти оценки были улучшены Э. И. Нечипоруком. В [79] им доказана следующая теорема.

ТеОРема 1.35. При любом фиксированном $\varepsilon>0 u n \rightarrow \infty$

$$
2^{n / 2}(1-\varepsilon)<\widetilde{L}_{k b}(n)<2^{n / 2+1} .
$$

А. А. Разборов в [80] доказал, что в любой контактно-вентильной схеме, реализующей произвольную симметрическую булеву функцию от $n$ переменных, не удовлетворяющую критерию линейности из [60; с. 105], содержится не менее $n \psi(n)$ контактов, где величина $\psi(n)$ пропорциональна количеству логарифмирований (по основанию 2 ) числа $n$, после которого впервые получается число, не превосходящее 1.

Из этого результата вытекает такая же нижняя оценка для числа контактов в минимальных контактных схемах, реализующих те же функции. 


\section{2. Булевы схемы}

2.1. Общие све́дения о булевых схемах. В настоящем разделе рассматриваются схемы, которые встречаются под названиями: схемы из функциональных элементов, комбинационные схемы, булевы схемы, схемы и т. п. В настоящем обзоре эти схемы называются булевыми схемами. Они определяются следующим образом.

Пусть $x_{1}, x_{2}, \ldots$ - булевы переменные, $B=\left\{E_{1}, \ldots, E_{r}\right\}$ - произвольный набор элементов, реализующих булевы функции. Набор $B$ называется базисом. Часто вместо элементов $E_{1}, \ldots, E_{r}$ указываются булевы функции $f_{1}, \ldots, f_{r}$ такие, что элемент $E_{i}$ реализует функцию $f_{i}$ от $n_{i}$ переменных (переменные функций не фиксируются).

Пусть $S$ - произвольный конечный ориентированный граф без параллельных дуг и контуров (т. е. ориентированных циклов), в котором некоторые вершины помечены символами из $B$, оставшиеся вершины помечены булевыми переменными $x_{1}, x_{2}, \ldots$, а все дуги, направленные в одну вершину, перенумерованы. При этом предполагается, что выполнены следующие требования:

1) отсутствуют дуги, направленные в вершины с метками $x_{1}, x_{2}, \ldots$;

2 ) число дуг, которые направлены в вершину с меткой $E_{i}$, равно $n_{i}, 1 \leqslant i \leqslant r$.

Такой граф $S$, в котором некоторые вершины используются как выходные, называется булевой схемой в базисе $B$. Вершины схемы $S$, помеченные символами $x_{1}, x_{2}, \ldots$, называются входами, вершины с метками из $B$ - элементами. Предполагается, что каждый элемент имеет только один выход, но если из некоторого элемента (вершины) исходит несколько дуг, то считается, что выход этого элемента подключен к нескольким входам других элементов. Все дуги схемы $S$ обычно интерпретируются как каналы, по которым происходит передача информации в направлении их ориентации. Входы схемы служат для ее подключения к источнику внешней информации, которая передается на входы схемы.

Нетрудно понять, что на выходе каждого элемента схемы $S$ вычисляется булева функция от тех переменных, которыми помечены входы схемы $S$.

Как обычно, полюсы (булевой) схемы изображаются маленькими кружочками, а элементы - треугольниками. При таком изображении отпадает необходимость в указании номеров каналов, если только условиться в том, что все каналы, направленные к элементу, подключены к одной стороне треугольника и следуют друг за другом слева направо, а выходом элемента является противоположная вершина треугольника.

В качестве примера на рис. 2.1 приведена булева схема, состоящая из дизъюнкторов $(V)$, конъюнкторов $(\&)$ и инвертора $\left(^{-}\right)$. На выходе этой схемы (помечен символом + ) вычисляется булева функция

$$
f\left(x_{1}, x_{2}, x_{3}\right)=\left(\left(x_{1} \vee x_{2}\right) \vee \overline{\left(x_{1} \& x_{2}\right)} \&\left(\left(x_{1} \& x_{2}\right) \vee x_{3}\right)=x_{1} x_{2} \bar{x}_{3} \vee x_{3}\right.
$$

2.2. Произвольные булевы схемы. В этом пункте освещаются результаты, относящиеся к поведению сложностной функции Шеннона, когда булевы функции из различных классов реализуются (вычисляются) произвольными булевыми схемами. 


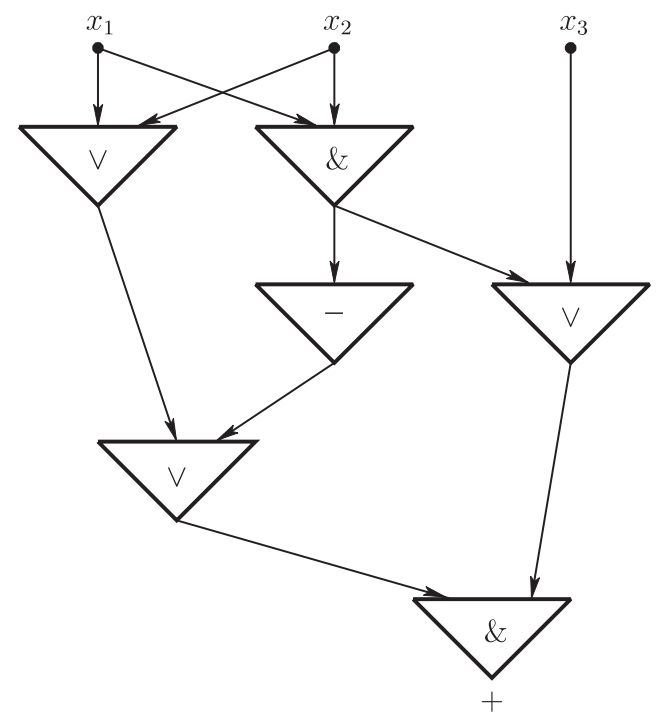

Рис. 2.1

Пусть $B=\left\{E_{1}, \ldots, E_{r}\right\}$ - произвольный конечный полный базис, и пусть элементу $E_{i}, 1 \leqslant i \leqslant r$, поставлено в соответствие положительное число $P\left(E_{i}\right)-$ сложность, или вес, элемента $E_{i}$ (содержательно это может быть цена изготовления или объем элемента и т. п.). Сложность произвольной схемы $S$ в базисе $B$ определяется как сумма стоимостей всех элементов в $S$ и обозначается через $L_{B}(S)$. Если стоимости всех элементов из $S$ в $B$ равны 1 , то под сложностью схемы $S$ в $B$ понимается число элементов в ней.

Пусть элемент $E_{i}, 1 \leqslant i \leqslant r$, вычисляет булеву функцию, существенно зависящую от $m_{i}$ переменных. Тогда величина

$$
\rho_{B}=\min \frac{P\left(E_{i}\right)}{m_{i}-1},
$$

где минимум берется по элементам $E_{i}$ из $B$ с $m_{i} \geqslant 2$, называется минимальным приведенным весом базиса $B$. Эту величину ввел в рассмотрение О. Б. Лупанов.

Пусть $H$ - произвольный класс булевых функций и $H(n)-$ совокупность функций из $H$ от переменных $x_{1}, \ldots, x_{n}$. Обозначим через $L_{B}(H(n))$ минимальное действительное число такое, что любая функция из $H(n)$ может быть вычислена булевой схемой $S$ в $B$, сложность которой не превосходит $L_{B}(H(n))$. Функцию $L_{B}(H(n))$ будем называть сложностной функцией Шеннона.

Ниже приводятся результаты, относящиеся к поведению сложностной функции Шеннона $L_{B}(H(n))$ для различных классов $H(n)$ и различных полных базисов $B$.

Если $H$ совпадает с множеством всех булевых функций, то вместо $L_{B}(H(n))$ используют обозначение $L_{B}(n)$.

Первый содержательный результат о поведении функции $L_{B}(n)$ содержится в работе Д. Мюллера [81]. Используя метод Шеннона, Д. Мюллер показал, что 
в случае произвольного конечного полного базиса $B$ справедливы неравенства

$$
c_{1} \frac{2^{n}}{n}<L_{B}(n)<c_{2} \frac{2^{n}}{n}
$$

где положительные константы $c_{1}, c_{2}$ зависят только от базиса $B$.

Дальнейшее существенное продвижение в исследовании функций $L_{B}(n)$ получил О. Б. Лупанов. В работе [31] им доказано следующее утверждение.

ТЕОРема 2.1. Пусть $B$ - произволъный конечный полный базис и $\rho_{B}-$ минимальный приведенный вес базиса $B$. Тогда при $n \rightarrow \infty$

$$
L_{B}(n) \sim \rho_{B} \frac{2^{n}}{n}
$$

причем среди булевых функиий от переменных $x_{1}, \ldots, x_{n}$ доля функций $f$ таких, что $L_{B}(f)<\rho_{B} \frac{2^{n}}{n}\left(1-\Omega\left(\frac{\log _{2} n}{n}\right)\right)$, стремится $\kappa$ нулю с ростом $n$.

Доказательство теоремы распадается на получение нижней и верхней оценок, асимптотически совпадающих между собой. Нижняя оценка вида $L_{B}(n)>$ $\rho_{B} \frac{2^{n}}{n}\left(1-\Omega\left(\frac{\log _{2} n}{n}\right)\right)$ устанавливается с привлечением мощностных соображений. Для получения верхней оценки О.Б. Лупанов ввел в рассмотрение специальное представление булевых функций, являющееся существенным обобщением обычного разложения булевых функций по подмножеству переменных. (Введенное представление часто называют представлением Лупанова.) Используя это представление, О.Б. Лупанов описал метод синтеза булевых схем в произвольном конечном полном базисе $B$, который для любой булевой функции от $n$ переменных позволяет строить схему сложности не более $\rho_{B} \frac{2^{n}}{n}\left(1+\Omega\left(\frac{\log _{2} n}{n}\right)\right)$.

Прежде чем перейти к освещению результатов, относящихся к поведению сложностной функции Шеннона для замкнутых классов булевых функций, введем в рассмотрение используемые (известные) понятия.

Пусть $A$ - некоторое множество булевых функций. Замыканием множества $A$ называется совокупность тех булевых функций, каждая из которых представима логической формулой над $A$. Замыкание множества $A$ обозначается через $[A]$. Класс $A$ булевых функций называется замкнутым, если $A=[A]$.

Э. Пост в [1] описал все замкнутые классы булевых функций. Часто эти классы называют замкнутыми классами Поста. Описание замкнутых классов Поста имеется в монографии [2]. Мы пользуемся терминологией и обозначениями из [2].

Булева функция $\varphi$ называется $\alpha$-функцией, если $\varphi(x, \ldots, x)=x, \beta$-функцией, если $\varphi(x, \ldots, x)=1$, и $\gamma$-функцией, если $\varphi(x, \ldots, x)=0$. Булева функция $\varphi\left(x_{1}, \ldots, x_{n}\right)$ называется самодвойственной, если $\varphi\left(x_{1}, \ldots, x_{n}\right)=\bar{\varphi}\left(\bar{x}_{1}, \ldots, \bar{x}_{n}\right)$.

Говорят, что булева функция $\varphi$ удовлетворяет условию $\left\langle a^{\infty}\right\rangle\left(\right.$ условию $\left.\left\langle A^{\infty}\right\rangle\right)$, если все наборы, на которых $\varphi$ равна нулю (единице), имеют общую нулевую (единичную) компоненту. 
Говорят, что булева функция $\varphi$ удовлетворяет условию $a^{\mu}$ (условию $\left\langle A^{\mu}\right\rangle$ ), если любые $\mu, \mu \geqslant 2$, наборов, на которых $\varphi$ равна нулю (единице), имеют общую нулевую (единичную) компоненту.

Теперь приведем известные результаты для замкнутых классов Поста, отличных от множества всех булевых функций (этот результат сформулирован в теореме 2.2).

Пусть $B=\left\{E_{1}, \ldots, E_{k}\right\}$ - произвольный конечный полный базис и $\rho_{B}-$ его минимальный приведенный вес.

А. Б. Угольников установил поведение функции $L_{B}(H(n))$ для всех замкнутых классов Поста. Подробное изложение этих результатов имеется в статье [82]. В ней сформулирован (в несколько ином виде) следующий результат.

ТеОрема 2.2. Пусть $B$ - произволъный конечный полный базис и $\rho_{B}-$ минимальный приведенный вес базиса $B$.

I. Если класс $H$ состоит из

1) самодвойственных булевых функиий (или $\alpha$-функицй);

2) функций (или $\alpha$-функиий), удовлетворяющих условию $\left\langle a^{\infty}\right\rangle$;

3) функиий (или $\alpha$-функиий), удовлетворяющих условию $\left\langle A^{\infty}\right\rangle$;

4) функиий (или $\alpha$-функиий), удовлетворяющих условию $\left\langle a^{\mu}\right\rangle, \mu=2,3, \ldots$;

5) бункций (или $\alpha$-функций), удовлетворяющих условию $\left\langle A^{\mu}\right\rangle, \mu=2,3, \ldots$, mo при $n \rightarrow \infty$

$$
L_{B}(H(n)) \sim \rho_{B} \frac{2^{n-1}}{n} .
$$

II. Если класс $H$ состоит из

1) монотонных функций, либо монотонных функций кроме 0, либо монотонных функиий кроме 1 , либо монотонных функиий кроме 0 и 1;

2) монотонных функиий (либо монотонных $\alpha$-функиий), удовлетворяющих условию $\left\langle a^{2}\right\rangle$;

3) монотонных функций (либо монотонных $\alpha$-функций), удовлетворяющих условию $\left\langle A^{2}\right\rangle$,

mo при $n \rightarrow \infty$

$$
L_{B}(H(n)) \sim \sqrt{\frac{2}{\pi}} \cdot \frac{2^{n}}{n^{3 / 2}} .
$$

III. Если класс H coстоит из

1) самодвойственных монотонных функций;

2) монотонных функций (или $\alpha$-функций), удовлетворяющих условию $\left\langle a^{\infty}\right\rangle$;

3) монотонных функиий (или $\alpha$-функиий), удовлетворяющих условию $\left\langle A^{\infty}\right\rangle$;

4) монотонных функций (или $\alpha$-функций), удовлетворяющих условию $\left\langle a^{\mu}\right\rangle$, $\mu=3,4, \ldots$;

5) функиий (или $\alpha$-функиий), удовлетворяющих условию $\left\langle A^{\mu}\right\rangle, \mu=3,4, \ldots$, mo при $n \rightarrow \infty$

$$
L_{B}(H(n)) \sim \rho_{B} \frac{2^{n}}{\sqrt{2 \pi} n^{3 / 2}} .
$$

IV. Если класс $H$ состоит из функиий любого другого замкнутого класса Поста, mo

$$
L_{B}(H(n))=O(n) .
$$


О. Б. Лупанов рассматривал задачу о сложности булевых схем в произвольном конечном полном базисе, вычисляющих степени отображений множества некоторых наборов из $E^{n}$ в себя, в следующей постановке.

Пусть $A=\left\{\widetilde{\sigma}_{1}, \ldots, \widetilde{\sigma}_{m}\right\}$ - некоторое множество наборов из $E^{n}$, и пусть $\mathscr{F}(n, A)$ - множество всех отображений наборов из $A$ в наборы из $A$. Каждое такое отображение может быть задано системой $n$ булевых функций $f_{1}\left(x_{1}, \ldots, x_{n}\right), \ldots, f_{n}\left(x_{1}, \ldots, x_{n}\right)$. Если $m<2^{n}$, то эти функции являются частичными.

Каждому отображению $F\left(x_{1}, \ldots, x_{n}\right)$ из $\mathscr{F}(n, A)$ ставится в соответствие вектор-функция $H_{F}\left(x_{1}, \ldots, x_{n}, y_{1}, \ldots, y_{n}\right)$ такая, что если набор $\widetilde{\sigma}=\left(\sigma_{1}, \ldots, \sigma_{n}\right)$ принадлежит множеству $A$, а набор $\widetilde{\tau}=\left(\tau_{1}, \ldots, \tau_{n}\right)$ - произвольный двоичный набор длины $n$, то

$$
H_{F}(\widetilde{\sigma}, \widetilde{\tau})=\underbrace{F(\ldots F(F(}_{t \text { раз }}(\tilde{\sigma})) \ldots),
$$

где $1 \leqslant t \leqslant 2^{n}$ и $\tau$ есть двоичная запись числа $t$.

Ясно, что если $m<2^{n}$, то вектор-функция $H_{F}\left(x_{1}, \ldots, x_{n}, y_{1}, \ldots, y_{n}\right)$ состоит из $n$ частичных булевых функций.

Пусть $B$ - произвольный конечный полный базис, каждому элементу которого приписан вес, и пусть $\rho_{B}$ - минимальный приведенный вес базиса $B$. Обозначим через $L_{B}^{*}(F)$ сложность простейшей (в смысле стоимости) булевой схемы в $B$ с $2 n$ входами и $n$ выходами, которая вычисляет $H_{F}\left(x_{1}, \ldots, x_{n}, y_{1}, \ldots, y_{n}\right)$.

Пусть $L_{B}^{*}(n, m)=\max L_{B}^{*}(F)$, где максимум берется по всем множествам $A$ из $m$ наборов длины $n$ и всем отображениям $F$ из $\mathscr{F}(n, A)$.

О. Б. Лупанов в [83] доказал следующий результат.

Теорема 2.3. Пусть $B$ - произвольный конечный полный базис, $\rho_{B}-$ его минимальный приведенный вес, и пусть $\frac{m}{\log _{2} n} \rightarrow \infty$ при $n \rightarrow \infty$. Тогда

$$
L_{B}^{*}(n, m) \sim \rho_{B} \frac{n m}{\log _{2}(n m)} .
$$

Нижняя оценка для $L_{B}^{*}(n, m)$ получается из мощностных соображений. Доказательство верхней оценки основано на применении метода локального кодирования Лупанова [84] и одного варианта теоремы Улига о сложности одновременного вычисления булевой функции на нескольких наборах (асимптотически без увеличения сложности). Используются также результаты Э. И. Нечипорука [78] и Л. А. Шоломова [85] (см. теорему 2), [86].

2.3. Булевы схемы с ограниченным ветвлением. Под булевой схемой с ограниченным ветвлением понимается такая булева схема, в которой выход каждого элемента соединен с небольшим числом входов других элементов. Такие схемы можно трактовать как схемы вычисления значений функций, в которых каждый промежуточный результат используется ограниченное число раз.

О. Б. Лупанов (см. теоремы 3 и 4 из [87]) для произвольного конечного полного базиса $B$ привел условия, при выполнении которых асимптотическое поведение сложностной функции Шеннона для сложности таких схем, реализующих 
булевы функции от $n$ переменных, совпадает с асимптотическим поведением функции $L_{B}(n)$ из теоремы 2.1 .

Сначала рассмотрим сложность булевых схем с ограниченным ветвлением в стандартном базисе $B_{0}=\left\{\vee, \&,{ }^{-}\right\}$. Пусть $L_{B_{0}}^{s}(n)$ обозначает минимальное натуральное число такое, что любая булева функция от $n$ переменных может быть реализована булевой схемой в стандартном базисе $B_{0}=\left\{\vee, \&,{ }^{-}\right\}$, в которой выход каждого элемента подсоединен не более чем к $s$ входам других элементов.

TEOPEMA 2.4. При $n \rightarrow \infty$

$$
L_{B_{0}}^{2}(n) \sim \frac{2^{n}}{n}
$$

Из теорем 2.1, 2.4 и очевидных неравенств

$$
L_{B_{0}}^{2}(n) \geqslant L_{B_{0}}^{3}(n) \geqslant \cdots \geqslant L_{B_{0}}^{s}(n)
$$

следует, что $L_{B_{0}}^{s}(n) \sim 2^{n} / n$ при любом $s \geqslant 2$.

Этот результат является окончательным, поскольку $L_{B_{0}}^{1}(n) \sim \frac{2^{n}}{\log _{2} n}($ см. теорему 2.1 при $\left.\rho_{B}=1\right)$.

Булевы схемы без ветвлений будут рассмотрены в разделе 3. Следует заметить, что такие схемы в России чаще всего называют либо формулами, либо суперпозициями. Название "булевы схемы без ветвлений" является, по-видимому, более удачным. В этом обзоре будем пользоваться этим названием.

В случае произвольного конечного полного базиса $B$ мы ограничиваемся частным видом условий Лупанова: выход каждого элемента из $B$ может быть подсоединен к такому числу входов других элементов, которое не превосходит числа его существенных входов. В этом случае асимптотическое поведение функции Шеннона для сложности таких схем не отличается от асимптотического поведения функции $L_{B}(n)$ из теоремы 2.1 .

2.4. Метод локального кодирования Лупанова. Многие универсальные методы синтеза булевых схем основаны на использовании метода локального кодирования, разработанного О. Б. Лупановым [84]. Этот метод мы будем называть методом локального кодирования Лупанова. Метод бесспорно относится к числу выдающихся достижений математики, и его идейное влияние испытывают на себе многие специалисты по синтезу схем.

Метод локального кодирования Лупанова для многих классов булевых функций позволяет получать существенно более простые схемы, чем в общем случае. Фактически этот метод путем некоторой перекодировки сводит вычисление функций из заданного класса к вычислению (произвольных) функций от меньшего числа переменных.

Метод локального кодирования Лупанова особенно удобен при синтезе схем, в которых допускается многократное использование промежуточных результатов. Для многих классов функций с применением этого метода удается получить асимптотику сложностной функции Шеннона. 
Суть метода локального кодирования Лупанова состоит в следующем. Все булевы функции из рассматриваемого класса кодируются последовательностями из нулей и единиц. Для построения асимптотически оптимального метода синтеза кодирование должно быть асимптотически оптимальным: длина кода должна быть асимптотически равна двоичному логарифму числа рассматриваемых функций от $n$ переменных. Кодирование должно быть локальным в том смысле, что для вычисления функции $f$ на каждом конкретном наборе $\sigma$ значений переменных достаточно знать сравнительно небольшой отрезок кода. Декодирование также должно осуществляться сравнительно просто. Сравнительно просто должна вычисляться координата отрезка кода, например, номер отрезка кода, если код разбит на отрезки одинаковой длины, номер первого разряда и длина отрезка, если отрезки имеют разную длину. По набору $\sigma$ и информации об отрезке кода сравнительно просто должно вычисляться значение $f(\sigma)$.

В общем случае схема вычисления, построенная для функции $f$ в соответствии с методом локального кодирования Лупанова, состоит из нескольких подсхем.

Первая подсхема по набору $\sigma$ вычисляет координаты отрезка кода. Вторая подсхема по координатам отрезка кода выдает часть кода, содержащего требуемый отрезок. Третья подсхема выделяет из части кода требуемый отрезок кода. Четвертая подсхема вычисляет $f(\sigma)$.

Обычно третья подсхема является универсальной, не зависит ни от класса реализуемых функций, ни от конкретной функции $f$. Первая и четвертая подсхемы не зависят от $f$. Вторая подсхема зависит от $f$ и содержит основную часть элементов всей схемы.

Метод локального кодирования Лупанова удобен, например, для синтеза схем, в которых многократно используются промежуточные результаты. Описание этого метода имеется в монографии [9; с. 124-148].

Применяя описанный метод, О. Б. Лупанов в [84] достаточно точно оценил значение сложностной функции Шеннона в следующих (и некоторых других) случаях.

1) Одновременное вычисление значений булевых функций от $n$ переменных на двух противоположных наборах. В этом случае асимптотика сложностной функции Шеннона равна $2^{n} / n$.

2) Одновременное вычисление значений булевых функций от $n$ переменных на нескольких последовательных наборах. В этом случае асимптотика сложностной функции Шеннона равна $2^{n} / n$.

3) Вычисление булевых функций от $n$ переменных из ненулевых инвариантных классов Яблонского. В случае, когда параметр инвариантного класса равен $\sigma$, асимптотика сложностной функции Шеннона равна $\sigma \cdot 2^{n} / n$.

4) Вычисление симметрических булевых функций от $n$ переменных. В этом случае сложностная функция Шеннона с точностью до порядка равна $n$.

$5)$ Вычисление булевых функций от $n$ переменных, принимающих единичное значение на $k_{n}$ наборах. Случай малых $k_{n}$ изучал Б. И. Фиников [88]. Из его результатов следует, что при $k_{n}=o\left(\log _{2} n\right)$ сложностная функция Шеннона 
с точностью до порядка равна $n$. О. Б. Лупанов показал, что если $k_{n}$ удовлетворяет условию $\lambda(n) \log _{2} n \leqslant k_{n} \leqslant 2^{n}-\lambda(n) \log _{2} n$, где $\lambda(n) \rightarrow \infty$ при $n \rightarrow \infty$, то асимптотика сложностной функции Шеннона в этом классе булевых функций равна

$$
\log _{2}\left(\begin{array}{l}
2^{n} \\
k_{n}
\end{array}\right) / \log _{2} \log _{2}\left(\begin{array}{l}
2^{n} \\
k_{n}
\end{array}\right) .
$$

Обобщения метода локального кодирования Лупанова имеются в работах Е. П. Липатова [89], [90]. Впоследствии этот метод применяли многие исследователи при исследовании сложности булевых схем, реализующих булевы функции из разных классов. Так, А. Б. Угольников (см. теорему 2.5 из [82]) доказал, что для булевых схем в произвольном конечном полном базисе $B$, вычисляющих монотонные булевы функции от $n$ переменных, сложностная функция Шеннона асимптотически равна $\rho_{B} \sqrt{\frac{2}{\pi n}} \cdot \frac{2^{n}}{n}$.

В работах Л.А. Шоломова [85] и Н. Пиппенджера [91] исследована сложность вычисления булевыми схемами частичных булевых функций; о таких функциях говорится в п. 2.5 .

2.5. Частичные булевы функции. Булева функция $f\left(x_{1}, \ldots, x_{n}\right)$, принимающая значения 0 и 1 на некотором собственном подмножестве из $E^{n}$, называется частичной (по другому, недоопределенной) булевой функцией.

Под доопределением частичной булевой функции от переменных $x_{1}, \ldots, x_{n}$ понимается всякая полностью определенная булева функция от переменных $x_{1}, \ldots, x_{n}$, совпадающая с исходной функцией в области ее определения.

Полагается, что булева схема, вычисляющая какое-либо доопределение частичной булевой функции $f$, вычисляет $f$.

Обозначим через $F(n, m)$ множество всех частичных булевых функций от переменных $x_{1}, \ldots, x_{n}$ таких, что $f$ определена на $m$ произвольных наборах из $E^{n}$.

Пусть $f\left(x_{1}, \ldots, x_{n}\right)$ - произвольная частичная булева функция, $B$ - произвольный конечный полный базис и $\rho_{B}$ - минимальный приведенный вес базиса $B$. Величина $L_{B}(f)=\min L_{B}(S)$, где минимум берется по всем булевым схемам $S$ в $B$, вычисляющим всевозможные доопределения частичной функции $f$ от переменных $x_{1}, \ldots, x_{n}$, называется сложностъю вычисления функции $f$ [92].

Далее, пусть $L_{B}(n, m)=\max L_{B}(f)$, где максимум берется по всем функциям $f$ из $F(n, m)$.

Используя метод локального кодирования Лупанова и оценки Нечипорука по сложности вентильных схем, Л. А. Шоломов в [85] (см. также [92]) получил следующий результат.

Теорема 2.5. Пусть последовательность натуралъных чисел $m_{1}, m_{2}, \ldots$ при некотором фиксированном $\gamma>0$ и $n \rightarrow \infty$ удовлетворяет условию

$$
\frac{m_{i}}{n\left(\log _{2} n\right)^{1+\gamma}} \rightarrow \infty
$$


Тогда при любом произвольном конечном полном базисе $B$

$$
L_{B}\left(n, m_{n}\right) \sim \rho_{B} \frac{m_{n}}{\log _{2} m_{n}},
$$

причем для каждого $\varepsilon, 0<\varepsilon<1$, доля функиий $f$ из $F(n, m)$ таких, что $L_{B}(f)<(1-\varepsilon) \frac{m_{n}}{\log _{2} m_{n}}$, стремится $\kappa$ нулю с ростом $n$.

При реальном синтезе булевых схем, вычисляющих булевы функции, часто используются методы, когда функции системы вычисляются последовательно и при вычислении очередной функции используются части схем, построенных для предыдущих функций.

Л.А. Шоломов в [93] исследовал асимптотическую сложность последовательного вычисления двух частичных булевых функций при растущем числе переменных. Установлено, что если области определения таких функций совпадают, то при их последовательном вычислении можно получить асимптотически наилучшую (в смысле сложности) булеву схему. При этом порядок вычислений не влияет на сложность схемы. В общем случае это не так. В частности, если область определения функции $f$ включает в себя область определения функции $g$, то при последовательном вычислении сначала функции $f$, а затем функции $g$ достижим асимптотически наилучший результат, а вычисление функций $f$ и $g$ в обратном порядке в общем случае приводит к повышению сложности всей схемы.

К этому направлению можно отнести работу [94]. В ней А. А. Семенов рассматривал приближенную реализацию булевых функций, которая заключается в следующем. Пусть для каждой булевой функции $f$ от $n$ переменных определена $\tau$-окрестность, состоящая из некоторых булевых функций от $n$ переменных и самой функции $f$. Скажем, что булева схема $S$ в стандартном базисе $\tau$-приближенно реализует функцию $f$, если $S$ реализует какую-либо функцию из $\tau$-окрестности функции $f$.

Под оптимальной сложностью $\tau$-приближенной реализации функции $f$ понимается число элементов из $B_{0}$, из которых можно построить булеву схему, реализующую некоторую функцию из $\tau$-окрестности функции $f$.

В работе [94] установлены асимптотики для сложностной функции Шеннона, которые зависят от размера и вида $\tau$-окрестностей.

2.6. Схемы из пороговых элементов. Булева функция называется пороговой, если множество ее единиц отделимо от множества ее нулей гиперплоскостью. Точнее говоря, булева функция $f\left(x_{1}, \ldots, x_{n}\right)$ называется пороговой, если существуют такие вещественные числа $w_{1}, \ldots, w_{n}, h$, что неравенство

$$
w_{1} x_{1}+\cdots+w_{n} x_{n} \leqslant h
$$

выполняется только при таких $x_{1}=\sigma_{1}, \ldots, x_{n}=\sigma_{n}$, когда $f\left(\sigma_{1}, \ldots, \sigma_{n}\right)=0$.

Ясно, что любая булева функция от переменных $x_{1}, \ldots, x_{n}$, принимающая значение 1 только на одном наборе, является пороговой. Однако такие булевы функции составляют исчезающе малую долю всех булевых функций от переменных $x_{1}, \ldots, x_{n}$. Вместе с тем любая булева функция $f$ от переменных 
$x_{1}, \ldots, x_{n}$, равная единице на $m$ наборах из $E^{n}$, очевидно, может быть задана системой таких линейных неравенств

$$
\left\{\begin{array}{c}
w_{11} x_{1}+\cdots+w_{1 n} x_{n} \leqslant h_{1}, \\
\cdots \ldots \ldots \ldots \ldots \ldots \ldots \ldots \ldots \\
w_{m 1} x_{1}+\cdots+w_{m n} x_{n} \leqslant h_{m}
\end{array}\right.
$$

что только нулевые наборы функции $f$ удовлетворяют этой системе.

Геометрически пороговое задание булевой функции $f$ сводится к отсечению гиперплоскостями всех вершин из $E^{n}$, на которых $f$ равна единице.

Под элементом, реализующим пороговую булеву функцию, заданную системой (2.2), понимается элемент с $n$ входами, на $i$-й вход которого подается значение переменной $x_{i}, 1 \leqslant i \leqslant n$, а на его выходе появляется нуль, если неравенства выполняются, и единица - в противном случае.

Схема, в которой все элементы являются пороговыми, называется схемой из пороговых элементов.

Нетрудно видеть, что любая булева функция $f\left(x_{1}, \ldots, x_{n}\right)$, заданная системой линейных неравенств (2.2), может быть реализована схемой из пороговых элементов $e_{1}, \ldots, e_{m}$ таких, что элемент $e_{i}, 1 \leqslant i \leqslant m$, реализует булеву функцию $f_{i}\left(x_{1}, \ldots, x_{n}\right)$, заданную $i$-м неравенством в $(2.2)$. Это означает, что на $j$-й вход элемента $e_{i}$ подается переменная $x_{j}$. K элементам $e_{1}, \ldots, e_{m}$ добавим еще один элемент $e$ c $m$ входами такой, что его $j$-й вход подключен к выходу элемента $e_{j}, 1 \leqslant j \leqslant m$, а на выходе элемента $e$ появляется 1 тогда и только тогда, когда на его входы поступает не менее одной единицы. Очевидно, элемент $e$ является пороговым и на его выходе реализуется функция $f\left(x_{1}, \ldots, x_{n}\right)$.

Из сказанного следует, что любая булева функция может быть реализована схемой из пороговых элементов.

Теперь изложим основные результаты, относящиеся к сложности схем из пороговых элементов, реализующих булевы функции (под сложностью схемы понимается число элементов в ней). Сначала заметим, что схемы из пороговых элементов строятся над бесконечным базисом, состоящим из всех пороговых элементов.

Пусть $f$ - произвольная булева функция. Обозначим через $L_{t}(f)$ число элементов в минимальной (по числу элементов) схеме из пороговых элементов, реализующей функцию $f$, и пусть $L_{t}(n)=\max L_{t}(f)$, где максимум берется по всем булевым функциям $f$ от $n$ переменных.

Сложность минимальных схем из пороговых элементов изучалась многими исследователями, например, Р. Уиндером, Л. А. Шоломовым, Н. П. Редькиным.

Так, например, Р. Уиндер показал, что

$$
L_{t}(n)=\Omega\left(\left(\frac{2^{n}}{n}\right)^{1 / 2}\right) .
$$

Более существенные результаты в этом направлении получили сначала Э. И. Нечипорук [95], а затем О. Б. Лупанов [96]. 
Э. И. Нечипорук установил следующие оценки для $L_{t}(n):$ при $n \rightarrow \infty$

$$
2\left(\frac{2^{n}}{n}\right)^{1 / 2}(1-o(1)) \leqslant L_{t}(n) \leqslant 2 \sqrt{2}\left(\frac{2^{n}}{n}\right)^{1 / 2}(1+o(1)) .
$$

Пусть $L_{t}(n, m)$ обозначает минимальное число пороговых элементов, достаточное для построения схемы из таких элементов, реализующей систему из $m$ произвольных булевых функций от переменных $x_{1}, \ldots, x_{n}$.

О. Б. Лупанов [96] установил асимптотическую формулу для $L_{t}(n, m)$ при некоторых естественных предположениях. Полученный результат формулируется следующим образом.

TeOpema 2.6. Пусть $m=o\left(2^{n}\right) u n \rightarrow \infty$. Тогда

$$
L_{t}(n, m) \sim 2\left(\frac{m \cdot 2^{n}}{n-\log _{2} m}\right)^{1 / 2} .
$$

В частности, при $m=1$ справедлива следующая теорема.

TEOpema 2.7. При $n \rightarrow \infty$

$$
L_{t}(n) \sim 2\left(\frac{2^{n}}{n}\right)^{1 / 2} .
$$

2.7. Булевы схемы в бесконечных базисах. В настоящем пункте рассматривается функция Шеннона, связанная со сложностью булевых схем. Поведение сложностной функции Шеннона в случае бесконечных базисов, когда под сложностью булевой схемы понимается число элементов в ней, гораздо разнообразнее, чем для конечных базисов, и пока неизвестен какой-либо общий метод, который бы позволял по произвольному бесконечному базису находить порядок роста соответствующей сложностной функции Шеннона. Вместе с тем известны примеры базисов, для которых порядки роста сложностной функции Шеннона равны: $1, \log _{2} n,\left(2^{n} / n\right)^{1 / 2}$ и $2^{n / 2}$. Они обнаружены в следующих работах.

Сложностная функция Шеннона, по порядку равная 1, найдена Н. А. Карповой. В работе [97] она показала, что если базис $B$ состоит из элементов, peaлизующих все булевы функции от $n-1$ переменного, то любая булева функция от $n$ переменных, $n \geqslant 4$, может быть вычислена схемой в $B$, содержащей три элемента.

Сложностная функция Шеннона, по порядку равная $\log _{2} n$, содержится в работах А. А. Маркова [98], [99].

Для схем из пороговых элементов, вычисляющих булевы функции от $n$ переменных, порядок сложностной функции Шеннона, равный 2, найден Э. И. Нечипоруком (см. (2.3)).

Помимо указанной информации О. М. Касим-Заде установил общую верхнюю оценку функции Шеннона, распространяющуюся на все бесконечные базисы: для каждого бесконечного полного базиса $B$ выполняется соотношение $L_{B}(n)<c \cdot 2^{n / 2}$, где $c-$ некоторая абсолютная (т. е. не зависящая от базиса) константа. Из результата Э.И. Нечипорука [95] следует, что эта оценка (с точностью до порядка) неулучшаема. 
В [100], [101] Н. А. Карпова описала необходимые и достаточные условия, которым должна удовлетворять функция натурального переменного, асимптотически равная функции Шеннона при вычислении булевых функций булевыми схемами в произвольных бесконечных базисах.

М. И. Гринчук в [102] доказал, что если базис $B$ состоит из элементов, peaлизующих все симметрические булевы функции, а вес каждого элемента равен числу его существенных входов, то $L_{B}(n) \sim 2^{n} / n$.

В работах [103], [104] О. М. Касим-Заде предложил новый подход к получению оценок сложности функций Шеннона для бесконечных базисов, позволяющий при некоторых несущественных ограничениях оценивать рост сложностной функкции Шеннона с точностью до полиномиальной эквивалентности.

К этому направлению можно отнести работу Н. А. Карповой [105] по синтезу булевых схем, вычисляющих булевы функции от переменных $x_{1}, \ldots, x_{n}$. Базис этих схем состоит из элементов, реализующих все булевы $(m, m)$-операторы, т. е. произвольные операторы, отображающие двоичные наборы длины $m$ в двоичные наборы той же длины. Предполагается, что $m \rightarrow \infty$ при $n \rightarrow \infty$ и $m<n$. Н. А. Карпова [105], [106] установила поведение функции Шеннона. Следует отметить, что это поведение зависит от величины параметра $m$, а такие схемы отличаются от обычных булевых схем.

2.8. Глубина булевых схем. Глубиной булевой схемы с одним выходом называется число элементов, принадлежащих самой длинной ориентированной цепи, в которой некоторый вход ее первого элемента является входом схемы, а выход ее последнего элемента является выходом схемы.

Пусть $f$ - произвольная булева функция и $B$ - базис. Булева схема в базисе $B$ минимальной глубины, реализующая функцию $f$, называется кратчайшей схемой для $f$ в базисе $B$. Глубина кратчайшей схемы для $f$ называется глубиной функции $f$ в $B$ и обозначается через $D_{B}(f)$.

Каждому базису $B$ ставится в соответствие функция $D_{B}(n)=\max D_{B}(f)$, где максимум берется по всем булевым функциям $f$ от $n$ переменных. Известно, что для любого конечного полного базиса $B$ асимптотика функции $D_{B}(n)$ при $n \rightarrow \infty$ имеет вид: $D_{B}(n) \sim n / \log _{2} m$, где $m$ - наибольшее число существенных переменных у функций базиса $B$.

В частности, различными авторами последовательно улучшались верхние оценки для $D_{B_{0}}(n)$ в случае стандартного базиса $B_{0}$. Наилучшая известная оценка содержится в [107]; она имеет вид $D_{B_{0}}(n) \leqslant n \log _{2} \log _{2} n-O(1)$.

Вопрос об описании поведения функции $D_{B}(n)$ для различных бесконечных полных базисов $B$ некоторое время оставался открытым.

Асимптотики или порядки были найдены лишь для небольшого числа базисов. При этом во всех известных примерах поведение функции $D_{B}(n)$ с точностью до постоянного множителя равна либо 1 , либо $\log _{2} n$. Например, если базис $B$ состоит из всех пороговых элементов, реализующих все булевы функции, то $D_{B}(n) \leqslant 2$ для любой булевой функции (этот факт следует из п. 2.6).

Следует заметить, что сложность таких схем, как правило, далека от сложности минимальных схем. 
Оказалось, что порядок функции $D_{B}(n)$ не может быть иным. Этот факт обнаружил О. М. Касим-Заде в работе [108], в которой была доказана следующая теорема.

ТЕОРема 2.8. Для любого бесконечного полного базиса В либо существует константа $\beta>1$ такал, что $D_{B}(n)=\beta$ при всех достаточно больших $n$, либо существуют целочисленные константы $\gamma \geqslant 2$ и $\delta$ такие, что

$$
\log _{\gamma} n \leqslant D_{B}(n) \leqslant \log _{\gamma} n+\delta
$$

В этой же работе найдены необходимые и достаточные условия, позволяющие отнести заданный бесконечный полный базис к одному из двух случаев теоремы 2.8 и для базисов, относящихся ко второму случаю, указать в явном виде значение константы $\gamma$.

2.9. Булевы схемы с задержками. В этом пункте рассматриваются булевы схемы с задержками, которые определяются следующим образом.

Пусть $B=\left\{E_{1}, \ldots, E_{s}\right\}$ - такой полный базис, что элемент $E_{i}, 1 \leqslant i \leqslant s$, реализует булеву функцию, существенно зависящую от $k_{i}$ переменных. Предполагается, что каждому элементу $E_{i}$ поставлено в соответствие положительное число $T_{i}$, называемое задержкой элемента $E_{i}$ (числа $T_{1}, \ldots, T_{s}$ не обязаны быть целыми).

Если $k_{i} \geqslant 2$, то число $T_{i} / \log _{2} k_{i}$ называется приведенной задержкой элемента $E_{i}$.

Булевы схемы с задержками отличаются от обычных булевых схем лишь тем, что в них элементы имеют задержки.

Задержкой цепи в булевой схеме с задержками называется сумма задержек тех элементов схемы, которые принадлежат этой цепи.

Цепь в булевой схеме с задержками называется главной, если некоторый вход ее первого элемента является входом схемы, а выход ее последнего элемента есть выход схемы.

Булева схема $S$ с задержками в базисе $B$ называется правильной, если задержки всех ее главных цепей одинаковы. Это общее число называется задержкой схемы $S$ и обозначается через $T_{B}(S)$.

Полный базис $B$ с задержками называется регулярным, если булевыми схемами в этом базисе можно реализовать с одной и той же задержкой все четыре функции от одной переменной $x, \bar{x}, 0,1$.

Мы будем использовать следующие обозначения:

$B=\left\{E_{1}, \ldots, E_{s}\right\}-$ регулярный полный базис;

$\tau=\min \left(T_{i} / \log _{2} k_{i}\right)$ - минимальная приведенная задержка базиса $B$, где минимум берется по всем таким $i$, что $k_{i} \geqslant 2$;

$L_{B}(f)$ - минимум сложностей правильных булевых схем в регулярном базисе $B$, реализующих булеву функцию $f$;

$L_{B}(n)=\max L_{B}(f)$, где максимум берется по всем булевым функциям $f$ от $n$ переменных;

$T_{B}(f)$ - минимум задержек правильных булевых схем в регулярном полном базисе $B$, реализующих булеву функцию $f$; 
$T_{B}(n)=\max T_{B}(f)$, где максимум берется по всем булевым функциям $f$ от $n$ переменных.

Введенные понятия заимствованы из работы [109]. В ней О. Б. Лупанов впервые получил важные результаты, относящиеся к правильным булевым схемам с задержками. Один из них формулируется следующим образом.

Теорема 2.9. Если $B=\left\{E_{1}, \ldots, E_{k}\right\}$ - регулярный базис с минималъным приведенным базисом $\rho_{B}$ и минимальной приведенной задержкой $\tau_{B}$, то

$$
L_{B}(n) \sim \rho_{B} \frac{2^{n}}{n}, \quad T_{B}(n) \sim \tau_{B} n .
$$

Более того, для любой булевой функции $f$ от $n$ переменных существует правильная булева схема в базисе В сложности не более $(1+\varepsilon) \rho_{B} \frac{2^{n}}{n}$ и с задержкой не более $(1+\varepsilon) \tau_{B} n(\varepsilon-$ произвольное положительное число), реализующая функиию $f$.

Различие и сходство между глубиной и задержкой булевых схем обсуждал В. М. Храпченко в [110], [111] и [112]. В этих же работах имеется несколько количественных соотношений для глубины и задержки.

2.10. Активность булевых схем. Известно несколько мер активности булевых схем. Рассмотрим две из них.

Пусть $S$ - булева схема с $n$ входами, и пусть на входы схемы $S$ поступает двоичный набор $\widetilde{\alpha}=\left(\alpha_{1}, \ldots, \alpha_{n}\right)$ значений переменных. В первом случае говорят, что элемент $E$ схемы $S$ является активным на наборе $\widetilde{\alpha}$, если при таком входном наборе выход элемента $E$ равен 1.

Во втором случае говорят, что элемент $E$ схемы $S$ является активным на наборе $\widetilde{\alpha}$, если при таком входном наборе по меньшей мере на один из входов элемента $E$ поступает 1 или выход элемента $E$ равен 1.

Далее в обоих случаях активность схемы $S$ определяется одинаково. А именно, обозначим через $\mathscr{A}(S, \widetilde{\alpha})$ число элементов схемы $S$, активных на входном наборе $\widetilde{\alpha}$. Величина

$$
\mathscr{A}(S)=\max \mathscr{A}(S, \widetilde{\alpha}),
$$

где максимум берется по всем входным наборам $\widetilde{\alpha}$ длины $n$, называется активностью схемы $S$.

Пусть $B$ - произвольный конечный полный базис, и пусть

$$
\mathscr{A}_{B}(f)=\min \mathscr{A}(S)
$$

где минимум берется по всем булевым схемам $S$ в базисе $B$, реализующим булеву функцию $f(\mathscr{A}(S)$ - активность схемы $S)$.

Наконец, пусть

$$
\mathscr{A}_{B}(n)=\max \mathscr{A}_{B}(f),
$$

где максимум берется по всем булевым функциям от переменных $x_{1}, \ldots, x_{n}$.

Функция Шеннона $\mathscr{A}_{B}(n)$ называется функцией активности булевых схем в базисе $B$, реализующих булевы функции от переменных $x_{1}, \ldots, x_{n}$. 
Впервые эту функцию ввел М. Н. Вайнцвайг. В работе [113] он изучал активность булевых схем в первом смысле. В этой работе им было показано, что поведение функции $\mathscr{A}_{B}(n)$ существенным образом зависит от базиса $B$. Кроме того, было установлено, что поведение функции $\mathscr{A}_{B}(n)$ полиномиально для одних $B$ и экспоненциально для других $B$. Вопрос о других поведениях функции $\mathscr{A}_{B}(n)$ остался открытым. Вместе с тем в случае базиса $B=\left\{\vee, \&,{ }^{-}\right\}$им был предложен такой метод синтеза булевых схем, который для любой булевой функции $f$ от $n$ переменных позволяет строить реализующую $f$ булеву схему активности не более $O(n)$, но, возможно, очень большого размера (в некоторых схемах содержится по $3 \cdot 2^{n}+n$ элементов).

Позднее О. М. Касим-Заде в [114] усилил последний результат, доказав следующее утверждение.

Теорема 2.10. Для любого фиксированного $\varepsilon>0$ и любой булевой функции $f$ от $n$ переменных существует такая булева схема $S$ в базисе $B_{0}=$ $\left\{\vee, \&,{ }^{-}\right\}$, реализующая $f$, что в схеме $S$ содержится не более $(1+\varepsilon) \frac{2^{n}}{n}$ элементов (ср. с теоремой 2.1) и ее активность не превосходит $(4+\varepsilon) n$.

Активность булевых схем во втором случае изучал О. М. Касим-Заде в работах [115], [116]. В них были получены следующие наиболее значительные результаты:

1) в зависимости от базиса $B$ функции $\mathscr{A}_{B}(n)$ бывают двух типов: полиномиальные и экспоненциальные;

2) найден эффективный критерий, позволяющий по конечному полному базису $B$ довольно просто устанавливать, какому из двух типов - полиномиальному или экспоненциальному - относится функция $\mathscr{A}_{B}(n)$.

\subsection{1. Неявная и параметрическая выразимость булевых функций.} Понятия неявной и параметрической выразимости булевых функций и функций $k$-значной логики при $k \geqslant 3$ были введены в рассмотрение А. В. Кузнецовым в [117] как обобщения понятия "задание функций формулами". Оба обобщения предполагают использование систем уравнений.

В случае неявной выразимости булевых функций каждая система уравнений состоит из некоторых формул над заданной совокупностью $B$ базисных функций (в базисе $B$ ), основных переменных и выделенной переменной.

Говорят, что система уравнений такого вида неявно задает булеву функцию $z=f\left(x_{1}, \ldots, x_{n}\right)$, если основными переменными системы являются $x_{1}, \ldots, x_{n}$, выделенной переменной - $z$ и при любых значениях переменных $x_{1}, \ldots, x_{n}$ система имеет единственное решение относительно $z$.

В случае параметрического задания булевых функций каждая система уравнений состоит из некоторых формул над заданной совокупностью $B$ базисных функций (в базисе $B$ ), основных и внутренних переменных, а также выделенной переменной.

Говорят, что система уравнений такого вида параметрически задает булеву функцию $z=f\left(x_{1}, \ldots, x_{n}\right)$, если основными переменными системы являются $x_{1}, \ldots, x_{n}$, внутренними переменными $-y_{1}, \ldots, y_{q}$, выделенной переменной $-z$, а при любых фиксированных значениях основных переменных система имеет 
по меньшей мере одно решение относительно $y_{1}, \ldots, y_{q}, z$, причем во всех таких решениях выделенная переменная $z$ равна одному и тому же значению.

Возможности параметрической выразимости булевых функций при различных базисах были установлены А. В. Кузнецовым в [117]. Затем О. М. Касим-Заде [118] показал, что в двухзначной логике неявная и параметрическая выразимости эквивалентны.

Перейдем к рассмотрению проблемы сложности неявной и параметрической выразимостей булевых функций.

Пусть $B$ - произвольный базис и $f$ - булева функция, неявно выразимая в $B$. Наименьшее число уравнений, достаточное для неявного задания функции $f$ в $B$, называется рангом в $B$ функции $f$ и обозначается через $m_{B}(f)$. Ранговой функцией в $B$ называется функция $m_{B}(n)=\max m_{B}(f)$, где максимум берется по всем булевым функциям $f$ от $n$ переменных, неявно выразимым в $B$ системами уравнений.

Пусть $B$ - произвольный базис и $f$ - булева функция, параметрически выразимая в $B$. Наименьшее число уравнений, достаточное для параметрического задания функции $f$ в $B$, называется $P$-рангом в $B$ функции $f$ и обозначается через $M_{B}(f)$. Функция $M_{B}(n)=\max M_{B}(f)$, где максимум берется по всем булевым функциям $f$ от $n$ переменных, параметрически выразимым в $B$ системами уравнений, называется $P$-ранговой функиией в $B$.

Поведение ранговых функций $m_{B}(n)$ и $M_{B}(n)$ изучал О. М. Касим-Заде [118]. Сначала он показал, что задача нахождения ранговых функций $m_{B}(n)$ и $M_{B}(n)$ для всех систем булевых функций $B$ сводится к нахождению ранговых функций $m_{B}(n)$ и $M_{B}(n)$ лишь для систем $B$, замкнутых относительно суперпозиций, т. е. замкнутых классов булевых функций (классов Поста). Затем для классов Поста им было доказано следующее утверждение (определение используемого в теореме обозначения $F_{i}^{\mu}$ имеется, например, в [2]).

Теорема 2.11. 1) Если замыкание системы B совпадает с классом всех монотонных булевых функиий, то при любом $n \geqslant 1$ справедливы равенства

$$
m_{B}(n)=\left\lfloor\frac{n+1}{2}\right\rfloor, \quad M_{B}(n)=2 .
$$

2) Если замыкание системы В совпадает либо с замкнутым классом таких монотонных булевых функиич, что функиия $f\left(x_{1}, \ldots, x_{n}\right)$ принадлежит этому классу тогда и только тогда, когда $f(x, \ldots, x)=x$ или $f(x, \ldots, x)=1$, либо с замкнутым классом таких монотонных булевых функций, что функиия $f\left(x_{1}, \ldots, x_{n}\right)$ принадлежит этому классу тогда и только тогда, когда $f(x, \ldots, x)=x$ или $f(x, \ldots, x)=0$, то при любом $n \geqslant 1$ справедливы равенства

$$
m_{B}(n)=\left\lfloor\frac{n+1}{2}\right\rfloor, \quad M_{B}(n)= \begin{cases}1 & \text { nрu } n=1 \\ 2 & \text { nрu } n \geqslant 2 .\end{cases}
$$

3) Если замыкание системы $B$ совпадает либо с замкнутым классом таких монотонных булевых функциц, что функиия $f\left(x_{1}, \ldots, x_{n}\right)$ принадлежит этому классу тогда и толъко тогда, когда $f(x, \ldots, x)=x$, то при любом $n \geqslant 1$ 
справедливы равенства

$$
m_{B}(n)=\left\lfloor\frac{n+1}{2}\right\rfloor, \quad M_{B}(n)= \begin{cases}1 & \text { nрu } n \leqslant 2, \\ 2 & \text { nрu } n \geqslant 3 .\end{cases}
$$

4) Если замыкание системь $B$ совпадает с одним из классов $F_{i}^{\mu}$, где $i=$ $1,4,5,8$ и $\mu=2,3, \ldots, \infty$, то при любом $n \geqslant 1$ справедливы равенства

$$
m_{B}(n)=\left\lfloor\frac{n+1}{2}\right\rfloor, \quad M_{B}(n)=\left\lceil\frac{n+1}{2}\right\rceil .
$$

5) Если замыкание системы В совпадает либо с классом самодвойственных монотонных булевых функиий, либо с одним из классов $F_{i}^{\mu}$, где $i=2,3,6,7$ и $\mu=2,3, \ldots, \infty$, то при любом $n \geqslant 1$ справедливы равенства

$$
m_{B}(n)=\Theta\left(n \log _{2} n\right), \quad M_{B}(n)=\Theta(n) .
$$

6) Если замыкание системы В совпадает с одним из оставиихся 28 классов Поста, то при любом $n \geqslant 1$ справедливы равенства

$$
m_{B}(n)=1, \quad M_{B}(n)=1 .
$$

2.12. Клеточные булевы схемы. До сих пор при рассмотрении булевых схем мы не учитывали, что реальные схемы размещаются в трехмерном пространстве, элементы имеют объем и соединяются между собой "проводниками", имеющими длину и толщину. Вместе с тем, интересен вопрос о сложности реализации булевых функций такими схемами. Эти схемы естественно называть пространственными (иначе, объемными) булевыми схемами. Формально их можно определить следующим образом.

Булева схема $S$ называется пространственной, если $S$ размещена в трехмерном пространстве так, что:

1) полюсы и элементы являются точками;

2) расстояние между любыми двумя точками не меньше 1;

3) проводники схемы $S$ суть линии, удовлетворяющие следующим ограничениям: расстояние между любыми двумя линиями, не инцидентными одной точке (т. е. полюсу или элементу), не меньше 1; если несколько линий инцидентны одной точке $a$, то вне шара минимально возможного диаметра с центром в точке $a$ расстояние между любыми линиями не меньше 1 (диаметр шара зависит от числа линий, инцидентных точке $a$; например, если точке $a$ инцидентны две линии, то диаметр шара равен 1).

Пусть каждому элементу базиса $B$ и единице длины проводника приписаны стоимости. Тогда под стоимостью пространственной булевой схемы в базисе $B$ с использованием проводников заданной стоимости понимается сумма стоимостей всех элементов и всех проводников в этой схеме.

Сложность пространственных булевых схем, как правило, существенно превосходит сложность обычных булевых схем. Это вытекает из теоремы 2.1 и следующего факта (теорема 2.12).

Пусть $B$ - конечный полный базис, и пусть $L_{B}(n)$ - функция Шеннона, которая при каждом $n$ равна такому минимальному действительному числу, что 
любую булеву функцию можно реализовать пространственной булевой схемой в базисе $B$, имеющей сложность, не большую величины $L_{B}(n)$.

Автор настоящего обзора в [119] доказал следующее утверждение.

Теорема 2.12. Пусть $B$ - произвольный конечный полный базис. Тогда nрu $n \rightarrow \infty$

$$
L_{B}(n)=\Omega\left(2^{n}\right) .
$$

Среди пространственных булевых схем особый интерес представляют плоские прямоугольные схемы из так называемых клеточных элементов (клеточные булевы схемы). Они аналогичны клеточным контактным схемам.
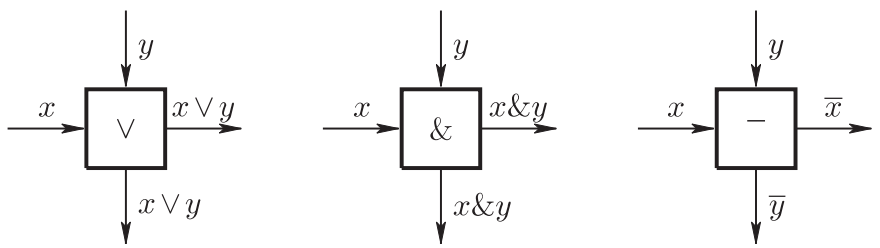

Рис. 2.2

Клеточная булева схема - это прямоугольник на плоскости, составленный из клеточных элементов. Клеточный элемент называется функциональным, если он реализует нетождественную функцию, и коммутационным, если он реализует одну или две тождественные функции. Ниже будут рассматриваться клеточные булевы схемы, в которых функциональные элементы реализуют дизъюнкцию $x \vee y$, конъюнкцию $x \& y$ и отрицание ${ }^{-}$(см. рис. 2.2). Кроме функциональных и коммутационных элементов в базисе имеется также изолирующий клеточный элемент без входов и выходов (см. рис. 2.3).
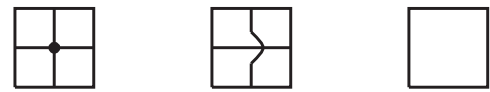

Рис. 2.3

Цепъю в клеточной булевой схеме называется всякая последовательность клеточных элементов, в которой выход каждого элемента, кроме последнего, соединен с одним из входов следующего элемента.

Перечислим наиболее значительные результаты о сложности клеточных булевых схем, реализующих различные булевы функции.

Обозначим через $L_{B_{0}}^{\mathrm{c}}(n)$ функцию Шеннона, которая при каждом $n$ равна такому минимальному натуральному числу, что любую булеву функцию от $n$ переменных можно реализовать клеточной булевой схемой в стандартном базисе $B_{0}=\left\{\vee, \&,{ }^{-}\right\}$, содержащей не более $L_{B_{0}}^{\mathrm{c}}$ элементов.

C. С. Кравцов в [120] впервые доказал следующее утверждение.

Теорема 2.13. Для любого фиксированного $\varepsilon>0$ u $n \rightarrow \infty$

$$
2^{n-2}(1-\varepsilon)<L_{B_{0}}^{\mathrm{c}}(n)<4.5 \cdot 2^{n}\left(1+\Omega\left(n \cdot 2^{-n / 2}\right)\right),
$$


причем среди булевых функиий от переменных $x_{1}, \ldots, x_{n}$ доля таких функиий, что они могут быть реализованы клеточными булевыми схемами сложности не более $2^{n-2}(1-\varepsilon)$, стремится $к$ нулю с ростом $n$.

Этот результат усилил А. Альбрехт в работе [121]. В ней доказана следующая теорема.

Теорема 2.14. Существует такое положительное число $\delta>0$, что

$$
L_{B_{0}}^{\mathrm{c}}(n) \sim \delta \cdot 2^{n} \quad \text { npu } \quad n \rightarrow \infty .
$$

В работе [122] Н. А. Шкаликова установила следующие факты.

ТЕОРема 2.15. 1) Площадь минимальной клеточной булевой схемъ в стандартном базисе $B_{0}$, реализующей все элементарные конбюнкиии от переменных $x_{1}, \ldots, x_{n}$, равна по порядку величине $n \cdot 2^{n}$.

2) Площадь минимальной клеточной булевой схемы в стандартном базисе $B_{0}$, реализующей все булевы функиии от переменных $x_{1}, \ldots, x_{n}$, равна по порядку величине $n \cdot 2^{2^{n}}$.

Кроме того, в [122] доказано, что площадь минимальной клеточной булевой схемы, реализующей умножение двух $n$-разрядных двоичных чисел, равна по порядку величине $n^{2}$. В этой же работе рассматривается задача о сложности реализации симметрических булевых функций клеточными булевыми схемами.

\subsection{3. Булевы схемы в базисах, содержащих элементы с нулевы-} ми весами. Задача синтеза булевых схем, содержащих элементы с нулевыми весами, формулируется следующим образом. Рассматриваются булевы схемы в базисе $B$, состоящем из элементов двух типов. Каждый элемент первого типа имеет положительную стоимость (вес), а каждый элемент второго типа - нулевую стоимость. Сложность схемы определяется как сумма весов (или число) элементов первого типа (ненулевая часть базиса $B$ ). Элементы второго типа не влияют на сложность схемы.

Задача состоит в построении булевой схемы как можно меньшей стоимости, реализующей заданную булеву функцию.

Для булевых схем такая постановка задачи впервые появилась в работе А. А. Маркова [98] относительно сложности булевых схем в стандартном базисе $B_{0}$, когда веса дизъюнктора и конъюнктора равны нулю, а вес инвертора единице. В ней была доказана следующая теорема.

Теорема 2.16. Любая булева функиия от $n$ переменных, $n \geqslant 1$, может быть реализована булевой схемой в стандартном базисе $B_{0}$, содержащем не более $\left\lceil\log _{2}(n+1)\right\rceil$ инверторов, причем эта верхняя оценка неулучшаема.

Позднее Э. И. Нечипорук в [123] получил еще несколько интересных результатов. Среди них упомянем следующие.

ТеОрема 2.17. Пусть базис В состоит из дизгюнктора и инвертора, вес дизгюнктора равен нулю, а вес инвертора - единище. Тогда любая булева функция от $n$ переменных может быть реализована булевой схемой в базисе $B$, 
содержащей не более $\sqrt{2^{n+1}}\left(1+\varepsilon_{n}\right)$ инверторов $(\varepsilon \rightarrow 0$ при $n \rightarrow \infty)$, причем среди булевых функиий от переменных $x_{1}, \ldots, x_{n}$ доля таких функиий $f$, что $f$ может быть реализована булевой схемой в базисе $B$, содержащей менее $\sqrt{2^{n+1}}\left(1-2 n / \sqrt{2^{n+1}}\right)$ инверторов, стремится $\kappa$ нулю с ростом $n$.

Теорема 2.18. Пусть базис В состоит из двух элементов, реализующих булевы функиии $f_{1}\left(x_{1}, x_{2}, x_{3}\right)=x_{1} \oplus x_{2} \oplus x_{3}$ u $f_{2}\left(x_{1}, x_{2}\right)=x_{1} x_{2}$, вес первого элемента равен нулю, а вес второго элемента - единище. Тогда любая булева функиия от $n$ переменных может быть реализована булевой схемой в базисе $B$, содержащей не более $2^{n / 2}\left(1+\varepsilon_{n}\right)$ конбюнкторов $(\varepsilon \rightarrow 0$ nри $n \rightarrow \infty)$, причем среди булевых функиий от переменных $x_{1}, \ldots, x_{n}$ доля таких функиий $f$, что $f$ может быть реализована булевой схемой в базисе $B$, содержащей менее $2^{n / 2}\left(1-3 n / 2^{n / 2}\right)$ конгюнкторов, стремится $к$ нулю с ростом $n$.

Заметим лишь, что изменение базиса при сохранении его нулевой части не существенно отражается на методах синтеза. При изменении же нулевой части базиса решение задачи требует в разных случаях существенно различных подходов.

\subsection{4. Полиномиальные нижние оценки сложности булевых схем,} реализующих конкретные булевы функции. Как и в случае контактных схем, проблема нижних оценок сложности булевых схем актуальна. Она состоит в том, чтобы научиться устанавливать "хорошие" нижние оценки для схем, вычисляющих конкретные булевы функции.

В случае произвольных булевых схем известны лишь линейные (относительно числа переменных вычисляемых функций) нижние оценки сложности минимальных схем. В случае неполных базисов известны более высокие нижние оценки.

Общее число известных нетривиальных нижних оценок сложности булевых схем, как и в случае контактных схем, невелико.

Некоторые сведения о таких оценках имеются в монографиях Р. Г. Нигматуллина [3], [6], Дж. Э. Сэвиджа [4], П. Данна [9] и обзорной статье В. М. Храпченко и работах других исследователей.

Среди оригинальных статей с такими оценками имеется несколько статей, в которых приведены точные значения числа элементов в минимальных булевых схемах. Перечислим основные из них.

1) Е. С. Горелик изучал сложность вычисления произвольных элементарных конъюнкций $x_{1}^{\sigma_{1}} \& \cdots \& x_{n}^{\sigma_{n}}$ булевыми схемами в базисе $\{\bar{x} \vee \bar{y}\}$. В работе [124] он показал, что в минимальной схеме такого типа, реализующей конъюнкцию $x_{1}^{\sigma_{1}} \& \cdots \& x_{n}^{\sigma_{n}}$, содержится $3 n-2-\sum_{i=1}^{n} \sigma_{i}$ элементов.

2) Г. А. Кочергина в [125] получила аналогичный результат для сложности минимальных булевых схем в других конкретных базисах.

3) К. П. Шнорр изучал сложность реализации булевых функций

$$
f\left(x_{1}, \ldots, x_{n}\right)=x_{1} \& \cdots \& x_{n} \vee \bar{x}_{1} \& \cdots \& \bar{x}_{n}, \quad n \geqslant 2,
$$

булевыми схемами в базисе, состоящем из всех одноместных и двухместных функций. В работе [126] он показал, что в минимальной схеме такого типа содержится $2 n-3$ элемента. 
4) Н. П. Редькин в [127] привел минимальные булевы схемы в стандартном базисе $B_{0}$, вычисляющие линейную булеву функцию от $n$ переменных (схема содержит $4 n-4$ элемента), оператор сравнения двух $n$-разрядных двоичных чисел (схема содержит $5 n-3$ элемента) и оператор совпадения двух $n$-разрядных двоичных чисел (схема содержит $5 n-1$ элемент). Далее, в [128] Н. П. Редькин показал, что минимальный $n$-разрядный двоичный сумматор в базисе, состоящем из всех булевых функций от двух переменных, содержит $5 n-3$ элемента.

5) И. С. Шкребела в [129] доказал, что в минимальной булевой схеме в базисе $\{x \rightarrow y, \bar{x}\}$, реализующей линейную булеву функцию от $n$ переменных, содержится $4 n-4$ элемента.

6) Э. И. Нечипорук в [130] получил первую нелинейную нижнюю оценку сложности минимальных булевых схем в базисе $\{\vee, \&\}$, реализующих систему таких $n$ дизъюнкций от $n$ переменных, что любые две дизъюнкции системы имеют не более одной общей переменной. Эта оценка равна $\Omega\left(n^{3 / 2}\right)$.

7) Булева схема называется синхронной, если во всех ее цепях, ведущих от входов к выходу, содержится одинаковое число элементов. Л. Харпер в [131] предложил метод получения нижних оценок сложности синхронных булевых схем, вычисляющих булевы функции. Затем в соавторстве с Дж. Э. Сэвиджем в [132] он упростил первоначальное доказательство и нашел применение предложенного метода к реальным синхронным схемам. На этом пути в [132] получена нижняя оценка $\Omega\left(n^{2} \log _{2} n\right)$ сложности минимальных синхронных булевых схем, вычисляющих булев детерминант бинарных квадратных матриц порядка $n$.

2.15. Высокие нижние оценки сложности булевых схем. Методы Разборова и Андреева. Изложенные в п. 2.14 нижние оценки для сложности булевых схем произвольного вида, вычисляющих конкретные булевы функции от $n$ переменных, линейны относительно $n$. Более высокие нижние оценки в общем случае пока неизвестны.

В сложившейся ситуации важно было бы понять, в какой мере трудности получения нелинейных оценок сложности вычисления конкретных булевых функций являются принципиальными.

При ответе на этот вопрос можно пойти на построение искусственного примера функции с высокой нижней оценкой сложности вычисления. Главное только в том, чтобы это способствовало разработке методов получения высоких нижних оценок для вычисления булевых функций, которые действительно представляют интерес.

В этом направлении было выполнено несколько работ. Например, Л. Мейер и Л. Стокмейер в 1972-1974 гг. указали путь построения сложно вычислимых булевых функций на базе формальных логических теорий. При этом символы кодируются двоичными наборами и в соответствии с этим кодируются предложения рассматриваемой теории. Оказывается, что булева функция $f$, принимающая значение 1 на кодах истинных предложений и значение 0 в остальных случаях, может оказаться очень сложно вычислимой. Это объясняется тем, что выразительные возможности средств формальной теории обычно настолько велики, что в ней можно описать довольно сложные утверждения, используя запись не очень большой длины. В таких случаях величина сложности вычисления булевой функции $f$ непосредственно в ее определение не входит. Трудно 
согласиться с конкретностью булевой функции $f$, для которой получена таким образом высокая нижняя оценка сложности вычислений.

Другим способом сложно вычислимые булевы функции построил Л. А. Шоломов [133], [134]. Его способ основан на определении значений булевой функции по начальным значениям рекурсивно-примитивных функций, имеющих начальные номера в некоторой вычислимой нумерации. Л.А.Шоломов показал, что сложность вычисления построенных им булевых функций может быть равна $c \cdot 2^{n} / n$, где $c$ - некоторая константа. В этом случае для задания булевой функции используются такие сильные вычислительные средства, как рекурсивно-примитивные функции.

K настоящему времени построено немало примеров сложно вычислимых булевых функций. Среди них отметим пример, построенный С. С. Марченковым B [135].

Перечисленные примеры сложно вычислимых булевых функций, по-видимому, не приближают нас к получению высоких нижних оценок сложности конкретных функций. Поэтому вопрос о существовании принципиального препятствия для получения нелинейных нижних оценок сложности остается открытым.

Существенное продвижение в получении высоких нижних оценок сложности для размера минимальных булевых схем в базисе $\{\vee, \&\}$, вычисляющих естественные монотонные булевы функции, произошло в 1985 г. А.А. Разборов в [136] установил сверхполиномиальные нижние оценки (по числу переменных) для размера минимальных булевых схем в базисе $\{\vee, \&\}$, которые вычисляют логический перманент булевых квадратных матриц и распознают наличие клики заданного размера в неориентированных графах.

Практически одновременно А.Е. Андреев в [137] установил квазиэкспоненциальные нижние оценки для размера подобных схем, вычисляющих менее естественные монотонные булевы функции. Эти функции обычно называют функциями Андреева. Хотя полученные А. А. Разборовым и А.Е. Андреевым оценки относятся к булевым схемам, вычисляющим разные булевы функции, и значительно разнятся по величине, методы, с помощью которых получены эти оценки, довольно похожи.

Сначала изложим результаты А. А. Разборова. В случае логического перманента булевых матриц точная постановка такова.

Пусть $A=\left(a_{i j}\right), 1 \leqslant i, j \leqslant n,-$ произвольная булева (двоичная) квадратная матрица порядка $n$. Логическим перманентом матрицы $A$ называется функция

$$
\operatorname{per} A=\underset{\sigma}{\bigvee} \underset{i=1}{\stackrel{n}{\&}} a_{i, \sigma(i)}
$$

где дизъюнкция берется по всем взаимно однозначным отображениям $\sigma$ : $\{1,2, \ldots, n\} \rightarrow\{1,2, \ldots, n\}$.

Пусть $\operatorname{PERM}\left(x_{1,1}, x_{1,2}, \ldots, x_{1, n}, x_{2,1}, x_{2,2}, \ldots, x_{2, n}, \ldots, x_{n, 1}, x_{n, 2}, \ldots, x_{n, n}\right)$ обозначает булеву функцию от $n^{2}$ переменных, которая на двоичном наборе

$$
\left(a_{1,1}, \ldots, a_{1, n}, a_{2,1}, \ldots, a_{2, n}, \ldots, a_{n, 1}, \ldots, a_{n, n}\right)
$$


равна 1 , если логический перманент матрицы $A=\left(a_{i j}\right)$ равен 1 , и равна 0 , если per $A=0$.

Легко видеть, что эта функция является монотонной. Поэтому она может быть вычислена булевой схемой в базисе $\{\vee, \&\}$ с $n^{2}$ входами. Обозначим через $L^{+}(\operatorname{PERM}(n))$ размер минимальной булевой схемы в базисе $\{\vee, \&\}$, которая вычисляет логический перманент булевых квадратных матриц порядка $n$.

А. А. Разборовым в [136] доказана следующая теорема.

ТЕОРЕма 2.19. При любом $n \geqslant 2$

$$
L^{+}(\operatorname{PERM}(n)) \geqslant n^{\left(\frac{1}{16}-\varepsilon_{n}\right) \log _{2} n},
$$

где $\varepsilon_{n} \rightarrow 0$ при $n \rightarrow \infty$.

Эта теорема утверждает, что логический перманент произвольной булевой матрицы не может быть вычислен булевой схемой в базисе $\{\mathrm{V}, \&\}$ с полиномиальным числом элементов относительно порядка матрицы. Вместе с тем из работы [138] следует, что логический перманент такой матрицы вычислим такой булевой схемой в стандартном базисе $B_{0}$, в которой содержится не более $\Omega\left(n^{3}\right)$ элементов. Таким образом, отношение размера минимальных булевых схем в базисе $\{\vee, \&\}$, вычисляющих логический перманент самых сложных квадратных матриц порядка $n$, к размеру аналогичных схем в стандартном базисе $B_{0}$, оказывается более чем полиномиальным относительно $n$.

Известно, что любая булева квадратная матрица $A$ порядка $n$ является матрицей смежности двудольного графа $G$, доли которого содержат по $n$ вершин, а логический перманент матрицы $A$ равен 1 тогда и только тогда, когда в $G$ имеется совершенное паросочетание. Поэтому вычисление перманента булевых квадратных матриц порядка $n$ и обнаружение совершенного паросочетания в соответствующих двудольных графах осуществимы одной и той же булевой схемой.

Задача о сложности распознавания наличия клики заданного размера в $m$-вершинных неориентированных графах состоит в следующем.

Пусть $\mathscr{G}(n)$ обозначает множество всех неориентированных графов с множеством вершин $V=\left\{v_{1}, \ldots, v_{m}\right\}$. Подмножество $V^{\prime}$ из $V$ называется кликой в графе $G \in \mathscr{G}(n)$, если любые две вершины из $V^{\prime}$ смежны в графе $G$. Число вершин в клике называется ее размером.

Двоичный набор $\left(a_{1,2}, \ldots, a_{1, m}, a_{2,3}, \ldots, a_{2, m}, \ldots, a_{2, m-1}, \ldots, a_{m-1, m}\right)$ длины $\left(\begin{array}{c}m \\ 2\end{array}\right)$ назовем кодом графа $G \in \mathscr{G}(n)$, если этот набор является таким, что

1) $a_{i, j}=1$, если в $G$ вершины $v_{i}$ и $v_{j}, i<j$, смежны;

2) $a_{i, j}=0$, если в $G$ вершины $v_{i}$ и $v_{j}$ несмежны.

Обозначим через $\operatorname{CLIQUE}_{s}\left(x_{1,2}, \ldots, x_{1, m}, x_{2,3}, x_{2,4}, \ldots, x_{i, j}, \ldots, x_{m-1, m}\right), 1 \leqslant$ $i, j \leqslant m$, такую булеву функцию от $\left(\begin{array}{c}m \\ 2\end{array}\right)$ переменных, что на двоичном наборе

$$
\left(a_{1,2}, \ldots, a_{1, m}, x_{2,3}, \ldots, a_{i, j}, \ldots, a_{m-1, m}\right)
$$

функция равна 1 , если этот набор является кодом графа, содержащим клику размера $s$, и функция равна 0, если этот набор является кодом графа, в котором нет клики размера $s$. 
Легко видеть, что введенная булева функция является монотонной. Поэтому она вычислима булевой схемой в базисе $\{\vee, \&\}$ с $\left(\begin{array}{c}m \\ 2\end{array}\right)$ входами.

Пусть $L^{+}(\operatorname{CLIQUE}(m, s))$ обозначает размер минимальной булевой схемы в базисе $\{\vee, \&\}$, которая вычисляет функцию

$$
\operatorname{CLIQUE}_{s}\left(x_{1,2}, \ldots, x_{1, m}, x_{2,3}, \ldots, x_{i, j}, \ldots, x_{m-1, m}\right) .
$$

А. А. Разборовым [136] доказана следующая теорема.

TeOPEma 2.20. Eсли $s=$ const, mo при $n \rightarrow \infty$

$$
L^{+}(\operatorname{CLIQUE}(m, s))=\Omega\left(\frac{m^{s}}{\left(\log _{2} m\right)^{2 s}}\right) .
$$

Eсли $s=\Theta\left(\log _{2} m\right)$, mo при $n \rightarrow \infty$

$$
L^{+}(\operatorname{CLIQUE}(m, s))=m^{\Omega\left(\log _{2} m\right)} .
$$

За цикл работ этого направления (см. [139]) А. А. Разборов получил премию Рольфа Неванлинны (Rolf Nevanlinna Prize) в 1990 г. на Международном конгрессе математиков в Киото.

При получении результатов в [137] А. Е. Андреев использовал сходные методы. В [137] он рассматривал последовательность менее естественных монотонных булевых функций и показал, что минимальные булевы схемы в базисе $\{\bigvee, \&\}$, которые вычисляют функции из этой последовательности, имеют квазиэкспоненциальный размер относительно числа переменных. Другая последовательность специальных монотонных булевых функций, которые также могут быть вычислены булевыми схемами в базисе $\{\vee, \&\}$, содержащими не менее чем квазиэкспоненциальное число элементов (не менее $2^{n^{\frac{1}{3}+\text { (1) }}}$ относительно числа переменных $n$ ), приведена в [137].

Для четкого описания результатов А.Е. Андреева требуются специальные понятия. Мы не будем на этом останавливаться. Отметим лишь, что в работе А. Е. Андреева используются другие понятия, нежели у А. А. Разборова. Для пояснения связи их результатов А. А. Разборов в [140] привел перевод понятий А. Е. Андреева на язык своих работ.

Техника доказательства, придуманная А. А. Разборовым, оказалась весьма эффективной. Она основана на использовании предложенного им метода аппроксимаций. Этот метод применим во многих случаях, хотя в каждой конкретной ситуации его использование требует отдельных весьма тонких рассмотрений.

Общий план метода аппроксимаций таков. Пусть требуется получить сверхполиномиальную или квазиэкспоненциальную нижнюю оценку для размера минимальной булевой схемы в базисе $\{\vee, \&\}$, вычисляющей некоторую булеву функцию $f$. Для этой цели рассматривается некоторый класс булевых функций, называемых "простыми", а также определяется специальное расстояние между двумя произвольными булевыми функциями. При этом расстояние от функции $f$ до любой простой функции сверхполиномиально или квазиэкспоненциально велико. Наряду с этим вводятся новые бинарные операции $\sqcap$ и 
"приближающие" операции \& и $\vee$ соответственно. Операции $\sqcap$ и $\sqcup$ обладают следующими свойствами. Если в схеме $S_{1}$, вычисляющей булеву функцию $f_{1}$, один конъюнктор (или дизъюнктор) заменить на элемент, реализующий операцию $\sqcap$ (или $\sqcup)$, то полученная схема будет вычислять такую булеву функцию $f_{2}$, что расстояние между $f_{1}$ и $f_{2}$ не превосходит 1 . Кроме того, после замены в исходной схеме всех конъюнкторов и дизъюнкторов на элементы $\square$ и $\sqcup$ соответственно получается схема, вычисляющая "простую" булеву функцию. Таким образом, расстояние между этой "простой” функцией и исходной функцией $f$ сверхполиномиально или квазиэкспоненциально велико. Следовательно, исходная схема $S$ имеет сверхполиномиальный или квазиэкспоненциальный размер.

Усовершенствовав комбинаторную технику доказательства из [136], Н. Алон и Р. Боппана усилили нижнюю оценку А. А. Разборова для размера минимальных булевых схем в базисе $\{\vee, \&\}$, которые распознают наличие клик размера $s$ в $m$-вершинных неориентированных графах. В работе [141] ими доказана следующая теорема.

Теорема 2.21. При любом фиксированном $s \geqslant 1 u m \rightarrow \infty$

$$
L^{+}(\operatorname{CLIQUE}(m, s))=\Omega\left(\frac{m^{s}}{\left(\log _{2} m\right)^{s}}\right) .
$$

Eсли $s=\Theta\left(\log _{2} m\right)$, то при $m \rightarrow \infty$

$$
L^{+}(\operatorname{CLIQUE}(m, s))=\exp \left\{\Omega\left(m^{1 / 6}\left(\log _{2} m\right)^{1 / 3}\right)\right\} .
$$

Подробное изложение результатов А. А. Разборова, А. Е. Андреева, Н. Алона и Р. Боппана имеется в монографии [9].

2.16. Разное. I. Сначала перечислим три верхние оценки сложности булевых схем, вычисляющих умножение двух произвольных $n$-разрядных чисел, заданных в двоичной системе счисления.

Распространенное и казавшееся правдоподобным мнение, что булева схема, осуществляющая такое умножение, основанное на школьном методе, близка к минимальной, опровергли в 1962 г. А. А. Карацуба и Ю. П. Офман. В [142] они описали булеву схему для умножения таких чисел, в которой содержится $O\left(n^{\log _{2} 3}\right)$ элементов.

Дальнейшие сюрпризы содержатся в заметке А. Л. Тоома [143]. В ней указана булева схема для умножения двух произвольных $n$-разрядных чисел, состоящая из $O\left(n \cdot 2^{c \sqrt{\log _{2} n}}\right)$ элементов.

В 1973 г. А. Шёнхаге и В. Штрассен в [144] описали метод умножения таких чисел, который можно реализовать посредством булевой схемы, содержащей $O\left(n \log _{2} n \cdot \log _{2} \log _{2} n\right)$ элементов. Этот метод основан на использовании быстрого преобразования Фурье.

II. Пусть $f\left(x_{1}, \ldots, x_{n}\right)$ - произвольная булева функция от $n$ переменных. Булева функция, полученная из $f\left(x_{1}, \ldots, x_{n}\right)$ в результате подстановки булевых констант $\alpha_{1}, \ldots, \alpha_{k}, 1 \leqslant k \leqslant n$, вместо переменных $x_{i_{1}}, \ldots, x_{i_{k}}$ соответственно, называется подфункиией функции $f$ от переменных из $\left\{x_{1}, \ldots, x_{n}\right\} \backslash$ 
$\left\{x_{i_{1}}, \ldots, x_{i_{k}}\right\}$. Если $\left\{y_{1}, \ldots, y_{m}\right\} \neq\left\{z_{1}, \ldots, z_{s}\right\}$, то подфункции $g\left(y_{1}, \ldots, y_{m}\right)$ и $h\left(z_{1}, \ldots, z_{s}\right)$ считаются разными. Число различных подфункций функции $f$ обозначим через $R(f)$ и назовем числом подфункций функции $f$.

Очевидно, что для любой булевой функции от $n$ переменных справедливо соотношение

$$
2^{n} \leqslant R(f) \leqslant 3^{n}
$$

С. В. Яблонский в [5] высказал предположение о том, что число подфункций булевой функции во многом определяет сложность ее реализации булевыми схемами. Оказывается, что это предположение справедливо не всегда. Это вытекает из следующих результатов.

1) А.Л. Тоом в [145] показал, что если булева функция $f$ от $n$ переменных имеет $k \cdot 2^{n}, 1 \leqslant k \leqslant(1.5)^{n}$, различных подфункций, то $f$ может быть вычислена булевой схемой в стандартном базисе $B_{0}$, содержащей не более $k^{2} n^{2}$ элементов.

2) Пусть $\mathscr{F}_{c, n}$ обозначает множество булевых функций $f\left(x_{1}, \ldots, x_{n}\right)$ таких, что $R(f)=c \cdot 2^{n}$, и пусть $B-$ произвольный конечный базис. Д. Улиг в [146] доказал, что в этом случае функция $f$ из $\mathscr{F}_{c, n}$ может быть реализована булевой схемой в базисе $B$, содержащей не более $\Omega(n)$ элементов.

3) В [147] Д. Улиг привел примеры таких булевых функций $f$ от $n$ переменных, что $R(f) \sim 3^{n}$ при $n \rightarrow \infty$ и функция $f$ может быть реализована булевой схемой в стандартном базисе $B_{0}$ с линейной (относительно $n$ ) сложностью.

III. Сумматор. Параллельным $n$-разрядным сумматором называется устройство, которое по цифрам $x_{1}, \ldots, x_{n}$ слагаемого $X$ и цифрам $y_{1}, \ldots, y_{n}$ слагаемого $Y$, поступающим одновременно на его входы, вычисляет цифры $z_{1}, \ldots, z_{n}$ их суммы $Z$. Предполагается, что числа $X, Y$ и $Z$ представлены в позиционной двоичной системе счисления.

Оператор, реализуемый параллельным $n$-разрядным сумматором, обозначим через $\Sigma_{n}$.

Будем считать, что такой сумматор реализуется булевой схемой с $2 n$ входами и $n+1$ выходом в произвольном конечном базисе $B$. Эту булеву схему обозначим через $S_{B}^{\Sigma_{n}}$, а ее глубину - через $D\left(S_{B}^{\Sigma_{n}}\right)$.

Так как старший разряд сумматора существенно зависит от всех $2 n$ разрядов слагаемых, а схема $S_{B}^{\Sigma_{n}}$ содержит не менее $n$ элементов из $B$, то глубина схемы $S_{B}^{\Sigma_{n}}$ по порядку не меньше $\log _{2} n$.

В.М. Храпченко подробно исследовал вопрос о глубине схем, реализующих $\Sigma_{n}$. Пусть $D_{B}\left(\Sigma_{n}\right)=\min D\left(S_{B}^{\Sigma_{n}}\right)$, где минимум берется по всем схемам в базисе $B$, реализующим оператор $\Sigma_{n}$.

В работе [148] он доказал следующее утверждение.

ТЕОРема 2.22. Для любого конечного полного базиса В существует константа $c_{B}, 0<c_{B} \leqslant 2$, такая, что

$$
D_{B}\left(\Sigma_{n}\right) \sim c_{B} \log _{2} n,
$$

причем можно указать базис $B$, для которого константа $c_{B}$ сколь угодно близка к 0, и можно указать базис $B$, для которого $c_{B}=2$.

В частности, для стандартного базиса $B_{0}$

$$
D_{B_{0}}\left(\Sigma_{n}\right) \sim \log _{2} n
$$


Н. П. Редькин рассматривал минимальную сложность реализации $n$-разрядного двоичного сумматора, состоящего из элементов, реализующих все булевы функции от двух переменных. В работе [128] им показано, что в таком сумматоре содержится $5 n-3$ элемента.

IV. В ряде работ изучалась возможность реализации булевых функций схемами на транзисторах, ламповых элементах и т. д. Например, Е. Ю. Захарова рассматривала схемы из ламповых элементов. Класс таких схем представляет собой некоторое расширение класса булевых схем, а именно, в этом классе допускается реализация булевых функций не только элементами, но и простым склеиванием проводников.

В [149] она доказала, что сложностная функция Шеннона $L(n)$ при реализации такими схемами в любом конечном полном базисе асимптотически равна $c \cdot 2^{n} / n$, где параметр $c$ определяется по базису.

\section{3. Булевы схемы без ветвлений}

3.1. Общие све́дения о булевых схемах без ветвлений. Среди булевых схем особый интерес представляют схемы, в которых выход каждого элемента подключен не более чем к одному входу какого-нибудь другого элемента. Такие схемы будем называть булевыми схемами без ветвлений (в литературе такие схемы часто называют формулами или суперпозициями).

Булевы функции, вычисляемые булевыми схемами без ветвлений, можно также задавать формулами, определяемыми индуктивно следующим образом.

Формулой в базисе $B$ называется:

1) символ любого литерала;

2) любое выражение вида $\varphi\left(F_{1}, \ldots, F_{k}\right)$, в котором $\varphi-$ символ $k$-местной булевой функции, принадлежащей базису $B$, а $F_{1}, \ldots, F_{k}$ - формулы в базисе $B$, есть формула.

Сложностъю формулы $F$ называется число вхождений символов из $B$ в формулу $F$.

Нетрудно видеть, что каждой формуле $F$, задающей булеву функцию $f$, можно поставить в соответствие такую булеву схему без ветвлений $S$, которая реализует функцию $f$ и имеет ту же сложность, что и формула $F$. Верно и обратное утверждение. Поэтому задача об определении сложности булевых схем без ветвлений в произвольном полном базисе $B$, реализующих булевы функции, эквивалентна задаче об определении сложности формул в базисе $B$, реализующих булевы функции.

Аналогичная связь имеется между контактными $\pi$-схемами и булевыми схемами без ветвлений в стандартном базисе $B_{0}$, поскольку между ними можно установить такое естественное взаимно однозначное соответствие, при котором соответствующие друг другу $\pi$-схема и булева схема без ветвлений реализуют одну и ту же булеву функцию и их сложности отличаются лишь на единицу. Различие может быть в том, что одни задачи удобнее решать на языке $\pi$-схем, а другие - на языке булевых схем без ветвлений или формул. 
Известно (см. [81]), что при переходе от одного конечного полного базиса к другому аналогичному базису стоимости минимальных булевых схем в этих базисах могут различаться не более чем в $c$ раз, где константа $c$ зависит только от базисов. Однако для булевых схем без ветвлений ситуация меняется.

В связи с этим обстоятельством отдельно рассматриваются булевы схемы без ветвлений, а для базисов были введены следующие понятия: отношение предшествования, строгого предшествования, эквивалентности, несравнимости и некоторые другие. Эти понятия определяются следующим образом.

Говорят, что базис $B_{1}$ предшествует базису $B_{2}$ (обозначение $B_{1} \preccurlyeq B_{2}$ ), если найдется такая константа $c$ (зависящая только от $B_{1}$ и $B_{2}$ ), что для любой булевой функции $f$ выполняется неравенство

$$
L_{B_{1}}^{F}(f) \leqslant c L_{B_{2}}^{F}(f)
$$

где $L_{B}^{F}(f)$ обозначает стоимость минимальной (по весу) булевой схемы без ветвлений в базисе $B$, реализующей булеву функцию $f$.

Базисы $B_{1}$ и $B_{2}$ называются эквивалентными (обозначение $B_{1} \sim B_{2}$ ), если выполнены оба соотношения $B_{1} \preccurlyeq B_{2}$ и $B_{2} \preccurlyeq B_{1}$. Если $B_{1} \preccurlyeq B_{2}$, а условие $B_{2} \preccurlyeq B_{1}$ не выполняется, то говорят, что базис $B_{1}$ cmpого предшествует базису $B_{2}$ (обозначение $B_{1} \prec B_{2}$ ).

Говорят, что базисы $B_{1}$ и $B_{2}$ несравнимы, если $B_{1} \npreceq B_{2}$ и $B_{2} \npreceq B_{1}$.

Говорят, что базис $B_{1}$ непосредственно предшествует базису $B_{2}$, если $B_{1} \prec B_{2}$ и не существует базиса $B$ такого, что $B_{1} \prec B$ и $B \prec B_{2}$.

Говорят, что булева функция $f$ реализуема бесповторно в базисе $B$, если существует булева схема без ветвлений, которая реализует $f$ и в которой каждый элемент из $B$ встречается не более одного раза.

Впервые эти понятия ввел в рассмотрение и изучал О. Б. Лупанов. Он доказал следующие утверждения.

1) если все функции, реализуемые элементами базиса $B_{1}$, бесповторно реализуемы в базисе $B_{2}$, то $B_{1} \preccurlyeq B_{2}$;

2 ) любой базис $B$ предшествует стандартному базису $B_{0}$, т. е. базис $B_{0}$ является самым плохим.

Затем существенные результаты получила Б.А. Субботовская (Мучник). Она доказала следующие утверждения.

1) Существуют неэквивалентные базисы. Например, базис $\left\{\vee, \&,{ }^{-}, \oplus, 1\right\}$ строго предшествует базису $B_{0}[150]$.

2) Произвольный конечный базис $B$ эквивалентен стандартному базису $B_{0}$ тогда и только тогда, когда все функции из базиса $B$ реализуемы бесповторно в $B_{0}[150]$.

3) Базис $B_{1}$ предшествует базису $B_{2}$ тогда и только тогда, когда существует такая константа $c>0$, что $L_{B_{1}}^{F}(f)<c L_{B_{2}}^{F}(f)$ для любой функции $f$ из специальной последовательности булевых функций [151].

4) Всякая булева схема без ветвлений в стандартном базисе $B_{0}$, вычисляющая линейную булеву функцию от $n$ переменных, содержит не менее $c n^{3 / 2}$ элементов, где $c>0$ - некоторая константа. Вместе с тем эта функция вычислима булевой схемой без ветвлений в базисе $B=\{x+y(\bmod 2)\}$, содержащей $\Omega(n)$ элементов. 
Д. Ю. Черухин рассматривал реализацию булевых функций булевыми схемами без ветвлений в базисе $B(k)$, где $B(k)$ состоит из всех булевых функций от $k$ переменных. В [152] он показал, что относительно сложности базис $B(k+1)$ существенно “лучше" базиса $B(k)$.

Отметим еще три результата Д. Ю. Черухина.

1) В [152] доказано существование счетной последовательности базисов, в которой каждый последующий базис строго предшествует предыдущему.

2) В [153] описано множество таких конечных полных базисов $B$, что линейные булевы функции можно реализовать булевыми схемами без ветвлений в базисе $B$ со сложностью $\Omega(n)$.

3) В [154] доказано, что если хотя бы одна функция из произвольного базиса $B$ не реализуема в стандартном базисе $B_{0}$ бесповторно, то сложность простейших булевых схем без ветвлений в базисе $B$, реализующих линейные булевы функции от $n$ переменных, по порядку меньше $n$.

3.2. Сложность булевых схем без ветвлений. Обозначим через $S_{B}^{F}(f)$ произвольную булеву схему без ветвлений в базисе $B=\left\{\varphi_{1}, \ldots, \varphi_{s}\right\}$, реализующую булеву функцию $f$. При этом предположим, что функции $\varphi_{i}, 1 \leqslant i \leqslant s$, поставлено в соответствие неотрицательное число $p_{i}$ - вес элемента, реализующего функцию $\varphi_{i}$. Под стоимостью булевой схемы $S_{B}^{F}(f)$ понимается сумма весов всех вхождений в $S_{B}^{F}(f)$ элементов, реализующих функции из $B$.

Обозначим через $L_{B}^{F}(f)$ стоимость минимальной булевой схемы без ветвлений в базисе $B$, реализующей булеву функцию $f$. Пусть $L_{B}^{F}(n)=\max L_{B}^{F}(f)$, где максимум берется по всем булевым функциям $f$ от $n$ переменных.

Первый существенный результат об асимптотическом поведении функции $L_{B}^{F}(n)$ в случае произвольного конечного полного базиса $B$ (с использованием результатов Р. Е. Кричевского из [155]) получил О.Б. Лупанов. В работе [32] им была доказана следующая теорема.

Теорема 3.1. Если веса всех элементов конечного полного базиса $B$, которые реализуют булевы функиии более чем от одной существенной переменной, положительны, то

$$
L_{B}^{F}(n) \sim \rho_{B} \frac{2^{n}}{\log _{2} n},
$$

где $\rho_{B}$ - минимальный приведенный вес базиса $B$ (см. п. 2.2).

Из этой теоремы следует, что функция $L_{B}^{F}(n)$ слабо зависит от базиса $B$ : она зависит только от $\rho_{B}$. Следствием теоремы 3.1 является асимптотика для $L_{B_{0}}^{F}(n)-$ сложности простейшей булевой схемы без ветвлений в стандартном базисе $B_{0}$, реализующей самую "сложную” булеву функцию от $n$ переменных, когда веса дизъюнктора и конъюнктора равны 1. Эта асимптотика имеет вид

$$
L_{B_{0}}^{F}(n) \sim \frac{2^{n}}{\log _{2} n} .
$$

А. Е. Андреев в [156] рассматривал реализацию булевыми схемами без ветвлений в произвольном конечном полном базисе $B$ булевых функций от $n$ переменных, принимающих единичное значение на $k_{n}$ наборах. В случае, когда 
$B=B_{0}$, а $k_{n}$ удовлетворяет условию $\log _{2} k_{n} \sim \log _{2}\left(2^{n}-k_{n}\right) \sim n$, он доказал, что асимптотика сложностной функции Шеннона в классе таких булевых функций равна

$$
\log _{2}\left(\begin{array}{l}
2^{n} \\
k_{n}
\end{array}\right) / \log _{2} n .
$$

Доказательство этого факта основано на применении метода локального кодирования Лупанова.

В работе [91] аналогичную задачу рассматривал Н. Пиппенджер в случае, когда булевы схемы без ветвлений в стандартном базисе $B_{0}$ реализуют такие частичные булевы функции $f$ от $n$ переменных, что $f$ определена на $d \cdot 2^{n}$ наборах и равна 1 на $p d \cdot 2^{n}$ наборах из $E^{n}$, где $p$ и $d$ - константы. Он доказал, что асимптотика сложностной функции Шеннона в классе таких частичных булевых функций равна

$$
\log _{2}\left(\begin{array}{c}
d \cdot 2^{n} \\
p d \cdot 2^{n}
\end{array}\right) / \log _{2} n
$$

Каждой формуле $F$ в стандартном базисе $B_{0}$ поставим в соответствие ранг, обозначаемый через $r(F)$. Определение ранга дадим по индукции.

Пусть $x_{1}, x_{2}, \ldots$ - булевы переменные. Тогда:

1) выражения $x_{i}$ и $\bar{x}_{j}$ являются формулами дизъюнктивного (и одновременно конъюнктивного) вида ранга 1 ;

2 ) если $F_{1}, \ldots, F_{s}-$ формулы ранга $0, s \geqslant 2$, то выражение $\left(F_{1}\right) \vee \cdots \vee\left(F_{s}\right)-$ формула дизъюнктивного вида ранга 1 ; а выражение $\left(F_{1}\right) \& \cdots \&\left(F_{s}\right)$ - формула конъюнктивного вида ранга 1;

3 ) если $F_{1}, \ldots, F_{s}$ - формулы конъюнктивного вида, $s \geqslant 2$, и среди них есть формула ранга не менее 1 , то выражение $\left(F_{1}\right) \vee \cdots \vee\left(F_{s}\right)$ есть формула дизъюнктивного вида ранга $1+\max \left\{r\left(F_{1}\right), \ldots, r\left(F_{s}\right)\right\}$;

4 ) если $F_{1}, \ldots, F_{s}$ - формулы дизъюнктивного вида, $s \geqslant 2$, и среди них есть формула ранга не менее 1 , то выражение $\left(F_{1}\right) \& \cdots \&\left(F_{s}\right)$ есть формула конъюнктивного вида ранга $1+\max \left\{r\left(F_{1}\right), \ldots, r\left(F_{s}\right)\right\}$.

Обозначим через $L_{B_{0}}^{F}(n, k)$ минимальное натуральное число такое, что любую булеву функцию от $n$ переменных можно задать такой формулой в стандартном базисе $B_{0}$, которая имеет ранг не более $k$ и содержит не более $L_{B_{0}}^{F}(n, k)$ вхождений символов из $B_{0}$.

Очевидно, что

$$
L_{B_{0}}^{F}(n, 2) \geqslant L_{B_{0}}^{F}(n, 3) \geqslant \cdots \geqslant L_{B_{0}}^{F}(n, k) \geqslant \cdots \geqslant L_{B_{0}}^{F}(n, \infty) .
$$

Из работы Дж. Риордана и К. Шеннона [30] следует, что (см. теорему 1.9)

$$
L_{B_{0}}^{F}(n, \infty) \geqslant \frac{2^{n}}{\log _{2} n}\left(1-\frac{3}{\log _{2} n}\right) .
$$


О. Б. Лупанов в [33] описал такой метод синтеза формул ранга 3, реализующих булевы функции от $n$ переменных, из которого извлекается следующая верхняя оценка для $L_{B_{0}}^{F}(n, 3)$ :

$$
L_{B_{0}}^{F}(n, 3)<\frac{2^{n}}{\log _{2} n}\left(1+\varepsilon_{n}\right),
$$

где $\varepsilon_{n} \rightarrow 0$ при $n \rightarrow \infty$.

Пользуясь неравенствами (3.1) и (3.3), получаем следующее утверждение.

Теорема 3.2. При любом биксированном $k \geqslant 3 u n \rightarrow \infty$ справедливо асимптотическое равенство

$$
L_{B_{0}}^{F}(n, k) \sim \frac{2^{n}}{\log _{2} n} .
$$

Этот результат является окончательным, поскольку $L_{B_{0}}^{F}(n, 2)=n \cdot 2^{n-1}-1$. Н. А. Карпова в [157] установила, какие числовые функции от $n$ переменных могут быть асимптотически равны сложностной функции Шеннона при реализации булевых функций булевыми схемами без ветвлений в произвольных полных базисах (как конечных, так и бесконечных).

3.3. Булевы схемы без ветвлений в базисах, содержащих элементы с нулевыми весами. Задача синтеза булевых схем в базисах, содержащих элементы с нулевыми весами, была сформулирована в п. 2.13. Для булевых схем без ветвлений эта задача формулируется аналогичным образом.

Основные результаты, относящиеся к сложности булевых схем без ветвлений в базисах, содержащих элементы с нулевыми весами, были получены Э. И. Нечипоруком в [158]. Сформулируем два из них.

Пусть $L_{B_{0}}^{-}(n)$ обозначает минимальное натуральное число такое, что любая булева функция от $n$ переменных может быть реализована булевой схемой без ветвлений в стандартном базисе $B_{0}$, содержащем не более $L_{B_{0}}^{-}(n)$ инверторов.

ТЕОРЕма 3.3. При любом натуральном $n$

$$
L_{B_{0}}^{-}(n)=\left\lceil\frac{n}{2}\right\rceil .
$$

Пусть $L_{B_{1}}^{-}(n)$ обозначает минимальное натуральное число такое, что любая булева функция от $n$ переменных может быть реализована булевой схемой без ветвлений в базисе $B_{1}=\left\{\mathrm{V},{ }^{-}\right\}$, содержащем не более $L_{B_{1}}^{-}(n)$ инверторов.

TEOPEMA 3.4. При $n \rightarrow \infty$

$$
L_{B_{1}}^{-}(n) \sim \frac{2^{n}}{n}
$$

3.4. Глубина и сложность булевых схем без ветвлений. Пусть $f-$ произвольная булева функция и $B$ - конечный полный базис. Глубина кратчайшей булевой схемы без ветвлений в базисе $B$, реализующая булеву функцию $f$, обозначается через $D_{B}^{F}(f)$. 
Базису $B$ ставится в соответствие числовая функция $D_{B}^{F}(n)=\max D_{B}^{F}(f)$, где максимум берется по всем булевым функциям $f$ от $n$ переменных. Ясно, что $D_{B}^{F}(n)=D_{B}(n)$ (функция $D_{B}(n)$ определена в п. 2.8).

В 1967 г. В. М. Храпченко (см. [159]) доказал следующее утверждение.

ТеОрема 3.5. Для любого конечного полного базиса В существуют такие константы с и $d$, что для всякой булевой функции $f$ справедливо неравенство

$$
D_{B}^{F}(f)<c \log _{2} L_{B}^{F}(f)+d
$$

Поскольку аналогичная нижняя оценка для $D_{B}^{F}(f)$ (с другими константами) получается тривиально, то оценка $(3.4)$ для $D_{B}^{F}(f)$ является с точностью до порядка наилучшей.

Позднее В. М. Храпченко в [159] доказал, что для стандартного базиса $B_{0}$ константа $c$ не превосходит 1.73 .

С. А. Ложкин в [160] убедился в справедливости следующего утверждения.

Теорема 3.6. Любую булеву функиию от $n$ переменных можно реализовать такой булевой схемой без ветвлений в стандартном базисе $B_{0}$, что при $n \rightarrow \infty$ ее сложность не превосходит $\frac{2^{n}}{\log _{2} n}\left(1+O\left(\frac{1}{\log _{2} n}\right)\right)$, а глубина - не более $n-\log _{2} \log _{2} n+o(1)$.

В 1970 г. О. Б. Лупанов [161] показал, что минимальные формулы различного ранга в одном и том же базисе, которые задают конкретную булеву функцию, могут существенно различаться по сложности. В качестве таких функций он рассмотрел последовательность булевых функций $f_{1}, f_{2}, \ldots$, где

$$
f_{n}=f_{n}\left(x_{1}, \ldots, x_{n} ; y_{1}, \ldots, y_{n}\right)=\bigvee_{i=1}^{n} x_{i} y_{i} y_{i+1} \cdots y_{n}
$$

и доказал следующее утверждение.

ТЕОРема 3.7. При любом фиксированном $d \geqslant 2 u n \rightarrow \infty$ сложнность минимальной формуль в базисе $B=\{\vee, \&\}$ ранга $d$, которая задает функцию $f_{n}$ из (3.5), асимптотически равна

$$
\frac{n(d-1)}{d}((d-1) ! n)^{1 /(d-1)} .
$$

3.5. Нижние оценки сложности булевых схем без ветвлений, вычисляющих конкретные булевы функции. Сначала рассмотрим методы получения нижних оценок сложности булевых схем без ветвлений в произвольных конечных полных базисах, вычисляющих индивидуальные булевы функции. $\mathrm{K}$ настоящему времени известны четыре метода: метод Нечипорука [57], метод Шпекера-Ходеса [58], метод Фишера-Мейера-Патерсона [162] и метод Черухина [163]. 
Метод НечипорукА. Э. И. Нечипорук в [57] описал такую булеву функцию от $n$ переменных, $n \geqslant 1$, которую нельзя вычислить булевой схемой без ветвлений в произвольном конечном базисе, содержащем менее $c n^{2} / \log _{2} n$ элементов.

Различные варианты доказательства теоремы Нечипорука, дающие разные значения константного множителя $c$, опубликованы и в других работах.

МЕТОД ШПЕКЕРА-ХоДЕСА. Этот метод получения нижних оценок сложности булевых схем без ветвлений в произвольных конечных полных базисах, вычисляющих булевы функции, описан в [58]. Он позволяет получить лишь невысокие нижние оценки: при заданном $n$ эти оценки по порядку равны $n \varphi(n)$, где $\varphi(n)$ равна количеству логарифмирований (по основанию 2) числа $n$, после которого впервые получается число, не превосходящее 1.

МЕТОД ФИШЕРА-МЕЙЕРА-ПАТЕРСОНА [162]. Метод Фишера-Мейера-Патерсона очень близок по духу к методу Шпекера-Ходеса. В случае базиса, состоящего из всех булевых функций от двух переменных, он позволяет получать нижние оценки сложности булевых схем без ветвлений, по порядку равные $n \log _{2} n$.

МЕтод ЧЕрухинА. Д. Ю. Черухин в [163] получил нижние оценки вида $\Omega\left(n \log _{2} n\right)$ сложности реализации почти всех симметрических булевых функций от $n$ переменных, в частности функции голосования, булевыми схемами без ветвлений в произвольном конечном полном базисе.

Другие методы получения нижних оценок сложности относятся к булевым схемам без ветвлений в стандартном базисе $B_{0}$. В этом базисе некоторые булевы функции (об этом было сказано выше) задаются значительно сложнее, чем могут быть заданы в других базисах (результат Д. Мюллера [81] о том, что все конечные полные базисы в случае булевых схем общего вида с точностью до порядка равноправны, на булевы схемы без ветвлений не распространяется).

Первый метод получения нелинейных нижних оценок сложности булевых схем без ветвлений в стандартном базисе $B_{0}$ предложила Б. А. Субботовская в [164]. Этим методом она установила первую нелинейную нижнюю оценку сложности реализации линейных булевых функций. Для таких функций от $n$ переменных эта оценка равна $c n^{3 / 2}$, где $c$ - подходящая не зависящая от $n$ константа (об этом говорилось в п. 3.1).

Оценка Субботовской впоследствии улучшалась с использованием других методов.

Второй метод получения нелинейных нижних оценок сложности предложил В. М. Храпченко [165]. Его формулировка для булевых схем без ветвлений в стандартном базисе $B_{0}$ эквивалентна формулировке в п. 1.7 для контактных $\pi$-схем.

В заключение отметим, что все нижние оценки сложности булевых схем, перечисленные в пп. 2.14 и 2.15, справедливы как нижние оценки сложности булевых схем без ветвлений. 
Выражаю искреннюю благодарность В. М. Храпченко за внимательное прочтение первоначального текста обзора, ценные замечания и предложения.

\section{Список литературы}

[1] E. L. Post, The two-valued iterative systems of mathematical logic, Ann. of Math. Stud., 5, Princeton Univ. Press, Princeton, NJ, 1941, viii+122 pp.

[2] С. В. Яблонский, Г. П. Гаврилов, В. Б. Кудрявцев, Функиии алгебры логики и классы Поста, Наука, М., 1966, 119 с.

[3] Р. Г. Нигматуллин, Сложность булевых функиий, Изд-во Казанского ун-та, Казань, 1983, 208 с.

[4] J. E. Savage, The complexity of computing, Wiley, New York-London-Sydney, 1976, хіii+391 рр.; рус. пер.: Д. Э. Сэвидж, Сложность вычислений, Факториал, М., 1998.

[5] С. В. Яблонский, "Об алгоритмических трудностях синтеза минимальных контактных схем", Проблемы кибернетики, 2, Физматгиз, М., 1959, 75-121.

[6] Р. Г. Нигматуллин, Сложность булевых функиий, Наука, М., 1991, 240 с.

[7] О.Б. Лупанов, Асимптотические оценки сложности управляющих систем, Изд-во МГУ, М., 1984, 136 с.

[8] I. Wegener, The complexity of Boolean functions, Wiley-Teubner Ser. Comput. Sci., Wiley, Chichester; Teubner, Stuttgart, 1987, xii+457 pp.

[9] P. E. Dunne, The complexity of Boolean networks, APIC Stud. Data Processing, 29, Academic Press, London, 1988, xii+490 pp.

[10] Р. Г. Нигматуллин, Нижние оценки сложности и сложность универсалъных схем, Изд-во Казанского ун-та, Казань, 1990, 112 с.

[11] P. Clote, E. Kranakis, Boolean functions and computation models, Texts Theoret. Comput. Sci. EATCS Ser., Springer-Verlag, Berlin, 2002, xiv+601 pp.

[12] W.H. Kautz, "A survey and assessment of progress in switching theory and logical design in the Soviet Union", IEEE Trans. Electronic Computers, EC-15:2 (1966), 164-204.

[13] Ю.Л. Васильев, В.В.Глаголев, “Метрические свойства дизъюнктивных нормальных форм", Дискретная математика и математические вопросы кибернетики, т. I, Наука, М., 1974, 99-148.

[14] M.S. Paterson, "An introduction to Boolean function complexity", Journées algorithmiques (École Norm. Sup., Paris, 1975), Astérisque, 38-39, 1976, 183-201.

[15] Ю.И. Журавлёв, "Булевых функций метрическая теория", Математическая энциклопедия, т. 1, Изд-во "Советская энциклопедия", М., 1977, 556-558.

[16] В. М. Храпченко, "Нижние оценки сложности схем из функциональных элементов", Кибернет. сб. Нов. сер., 21, Мир, М., 1984, 3-54.

[17] А.А. Сапоженко, И.П. Чухров, "Минимизация булевых функций в классе дизъюнктивных нормальных форм", Итоги науки и техн. Теор. вероятн. Матем. статист. Теорет. кибернет., 25, ВИНИТИ, М., 1987, 68-116; англ. пер.: A. A. Sapozhenko, I. P. Chukhrov, "Boolean function minimization in the class of disjunctive normal forms", J. Soviet Math., 46:4 (1989), 2021-2052.

[18] А. Д. Коршунов, “Монотонные булевы функции”, УМН, 58:5(353) (2003), 89-162; англ. пер.: A. D. Korshunov, "Monotone Boolean functions", Russian Math. Surveys, 58:5 (2003), 929-1001.

[19] C. E. Shannon, "A symbolic analysis of relay and switching circuits", Trans. Amer. Inst. Electrical Engineers, 57:12 (1938), 713-723; рус. пер.: К. Шеннон, "Символический анализ релейных и переключательных схем", К. Шеннон. Работы по теории информачии и кибернетике, ИЛ, М., 1963, 9-45. 
[20] C. Shannon, "The synthesis of two-terminal switching circuits", Bell System Techn. J., 28:1 (1949), 59-98; pyc. пер.: К. Шеннон, "Синтез двухполюсных переключательных схем", К. Шеннон. Работы по теории инбормации и кибернетике, ИЛ, М., 1963, 59-113.

[21] Г. Н. Поваров, "Математическая теория синтеза контактных $(1, k)$-полюсников", Докл. АН СССР, 100:5 (1955), 909-912.

[22] Г.Н. Поваров, "Метод синтеза вычислительных и управляющих контактных схем", Автомат. и телемех., 18:2 (1957), 145-162.

[23] О.Б. Лупанов, “О синтезе некоторых классов управляющих систем”, Проблемы кибернетики, 10, Физматгиз, М., 1963, 63-97.

[24] E. F. Moore, "Minimal complete relay decoding networks", IBM J. Res. and Develop., 4:5 (1960), 524-531; рус. пер.: Э.Ф. Мур, "Минимальные полностью декодирующие контактные схемы”, Кибернет. сб., 6, ИЛ, М., 1963, 139-152.

[25] А. Д. Коршунов, "О нижних оценках сложности контактных схем, реализующих попарно ортогональные функции алгебры логики", Дискретный анализ, 2, Ин-т математики СО АН СССР, Новосибирск, 1964, 42-47.

[26] Н. П. Редькин, "О реализации систем конъюнкций контактными схемами”, Проблемы кибернетики, 30, Наука, М., 1975, 263-276.

[27] С.А. Ложкин, М.А. Кошкин, "О сложности реализации некоторых систем функций алгебры логики контактными и обобщенными контактными схемами”, Математические вопросы кибернетики, 3, Физматлит, М., 1991, 257-285.

[28] Х.А. Мадатян, "Синтез контактных схем ограниченной ширины”, Проблемы кибернетики, 14, Наука, М., 1965, 301-307.

[29] С. А. Ложкин, "О синтезе ориентированных контактных схем", Вестн. Моск. ун-та. Сер. 15. Вычислит. матем. и кибернет., 1995, №2, 36-42; англ. пер.: S. A. Lozhkin, "On the synthesis of oriented switching circuits", Moscow Univ. Comput. Math. Cybern., 1995, № 2, 32-37.

[30] J. Riordan, C. E. Shannon, "The number of two-terminal series-parallel networks", J. Math. Phys. Mass. Inst. Tech., 21:2 (1942), 83-93; рус. пер.: Дж. Риодан, К. Шеннон, “Число двухполюсных параллельно-последовательных сетей”, К. Шеннон. Работы по теории информации и кибернетике, ИЛ, М., 1963, 46-58.

[31] О.Б. Лупанов, "Об одном методе синтеза схем", Изв. вузов. Радиофизика, 1:1 (1958), 120-140.

[32] О. Б. Лупанов, "О сложности реализации функций алгебры логики формулами", Проблемы кибернетики, 3, Физматгиз, М., 1960, 61-80.

[33] О.Б. Лупанов, "О реализации функций алгебры логики формулами ограниченной глубины в базисе $\&, \vee,{ }^{-}$, , Проблемы кибернетики, 6, Физматгиз, М., 1961, $5-14$.

[34] Е. А. Кондратьева, "Об универсальных $\pi$-сетях для функций алгебры логики от $n$ переменных", Проблемы кибернетики, 14, Наука, М., 1965, 5-16.

[35] О. А. Задорожнюк, "О контактных схемах из клеточных элементов", Математические вопросы кибернетики, 6, Физматлит, М., 1996, 257-280.

[36] О. Б. Лупанов, "О сложности универсальной параллельно-последовательной сети глубины 3", Математическая логика, теория алгоритмов и теория множеств, Сборник работ. Посвящается академику Петру Сергеевичу Новикову к его семидесятилетию, Тр. МИАН СССР, 133, 1973, 127-131; англ. пер.: O.B. Lupanov, "The complexity of the universal parallel-sequential network of depth 3", Proc. Steklov Inst. Math., 133 (1977), 127-132.

[37] Е. В. Шеришева, "Построение универсальных контактных схем", Проблемы кибернетики, 18, Наука, М., 1967, 303-312.

[38] Ф.Я. Ветухновский, "Об оценках числа плоских графов", Докл. АН СССР, 142:1 (1962), 50-53. 
[39] А. Д. Коршунов, "Об асимптотических оценках сложности некоторых классов контактных схем", Кибернетика, 1965, № 2, 18-28.

[40] Ю.Г. Таразевич, "О сложности реализации многополюсников плоскими контактными схемами", Вестн. Моск. ун-та. Сер. 1. Матем., мех., 1992, № 6, 59-61; англ. пер.: Yu. G. Tarazevich, "On the complexity of the realization of multipoles by means of plane contact circuits", Moscow Univ. Mech. Bull., 47:6 (1992), 20-22.

[41] А.К. Пулатов, "О влиянии нулевых цепей на сложность реализации булевых функций контактными схемами", Методы дискретного анализа в решении комбинаторных задач, Дискретный анализ, 30, Ин-т математики СО АН СССР, Новосибирск, 1977, 30-37.

[42] С. В. Здобнов, "О сложности линейной функции в классе $\pi$-схем без нулевых цепей”, Комбинаторно-алгебраические методы и их применение, Горьковский ун-т, Горький, 1987, 27-34.

[43] С. В. Здобнов, "Алгоритм синтеза минимальных $\pi$-схем без нулевых цепей, реализующих характеристические функции линейных кодов", Вероятностные методы и кибернетика, 23, Изд-во Казанского ун-та, Казань, 1987, 87-97.

[44] С. В. Здобнов, "О сложности реализации кодовых функций в классе контактных схем без нулевых цепей", Методы дискретного анализа в исследовании функциональных систем, Методы дискретного анализа, 47, Ин-т математики СО АН СССР, Новосибирск, 1988, 47-60.

[45] С. В. Здобнов, "Нижняя оценка функции Шеннона для контактных схем без нулевых цепей", Методы дискретного анализа в изучении булевых функиий и графов, Методы дискретного анализа, 48, Ин-т математики СО АН СССР, Новосибирск, 1989, 3-16.

[46] С. Е. Кузнецов, "Схемы из функциональных элементов без нулевых цепей в базисе $\{\&, \vee,-\} ”$, Изв. вузов. Матем., 1981, № 5, 56-63; англ. пер.: S. E. Kuznetsov, "Circuits of functional elements without zero chains in the basis $\{\&, \vee,-\}$ ", Soviet Math., 25:5 (1981), 62-73.

[47] С. Е. Кузнецов, "Нижняя оценка функции Шеннона для $\pi$-схем без нулевых цепей", Методы дискретного анализа в изучении булевых функций и графов, Методы дискретного анализа, 37, Ин-т математики СО АН СССР, Новосибирск, 1981, 51-64.

[48] И.О. Соколов, "Верхние оценки сложности реализации характеристических функций групповых кодов контактными схемами без нулевых цепей", Дискрет. матем., 7:1 (1995), 110-122; англ. пер.: I. O. Sokolov, "Upper bounds on the complexity of the realization of characteristic functions of group codes by switching circuits without zero chains", Discrete Math. Appl., 5:2 (1995), 137-148.

[49] А. Д. Коршунов, "Об асимптотических оценках сложности контактных схем заданной степени", Дискретный анализ, 5, Ин-т математики СО АН СССР, Новосибирск, 1965, 35-67.

[50] В.М. Храпченко, "О сложности реализации линейной функции в классе П-схем", Матем. заметки, 9:1 (1971), 35-40; англ. пер.: V. M. Khrapchenko, "Complexity of the realization of a linear function in the class of П-circuits", Math. Notes, 9:1 (1971), 21-23.

[51] С. В. Яблонский, "Реализация линейной функции в классе $\pi$-схем”, Докл. АН СССР, 94:5 (1954), 805-806.

[52] К. Л. Рычков, "Модификация метода В. М. Храпченко и применение ее к оценкам сложности $\pi$-схем для кодовых функций", Методы дискретного анализа в теории графов и схем, Методы дискретного анализа, 42, Ин-т математики СО АН СССР, Новосибирск, 1985, 91-98. 
[53] О.Б. Лупанов, "К вопросу о реализации симметрических функций алгебры логики контактными схемами", Проблемы кибернетики, 15, Наука, М., 1965, 85-99.

[54] C. Cardot, "Quelques résultats sur l'application de l'algèbre de Boole à la synthèse de circuits à relais", Ann. Télécommun., 7:2 (1952), 75-84.

[55] С. М. Вартанян, "Новое доказательство минимальности контактной схемы, реализующей линейную функцию", Методы дискретного анализа в изучении реализаиий логических функиий, Методы дискретного анализа, 41, Ин-т математики СО АН СССР, Новосибирск, 1984, 27-34.

[56] 3. Е. Королева, "Доказательство минимальности контактных схем некоторого типа", Дискретный анализ, 14, Ин-т математики СО АН СССР, Новосибирск, 1969, 18-27.

[57] Э. И. Нечипорук, “Об одной булевской функции”, Докл. АН СССР, 169:4 (1966), 765-766; англ. пер.: Eh. I. Nechiporuk, "A Boolean function", Soviet Math. Dokl., 7 (1966), 999-1000.

[58] L. Hodes, E. Specker, "Lengths of formulas and elimination of quantifiers. I", Contributions to mathematical logic (Hannover, 1966), North-Holland, Amsterdam, 1968, 175-188; рус. пер.: Л. Ходес, Е. Шпекер, “Длины формул и исключение кванторов”, Кибернет. сб. Нов. сер., 10, Мир, М., 1973, 99-113.

[59] А. Е. Андреев, "Об одном методе получения более чем квадратичных эффективных нижних оценок сложности $\pi$-схем", Вестн. Моск. ун-та. Сер. 1. Матем. мех., 1987, № 1, 70-73; англ. пер.: А. E. Andreev, "A method for obtaining more than quadratic effective lower estimates of complexity of $\pi$ schemes", Moscow Univ. Math. Bull., 42:1 (1987), 63-66.

[60] М.И. Гринчук, "О сложности реализации симметрических булевых функций контактными схемами", Математические вопросы кибернетики, 3, Физматлит, M., 1991, 77-114.

[61] М. И. Гринчук, "Нижняя оценка сложности реализации симметрических булевых функций контактными схемами", Математические вопросы кибернетики, $\mathbf{4}$, Физматлит, М., 1992, 130-138.

[62] Ю. И. Журавлёв, “Об отделимости подмножеств вершин $n$-мерного единичного куба", Сб. статей по математической логике и ее приложениям к некоторым вопросам кибернетики, Изд-во АН СССР, М., 1958, 143-157.

[63] Ю.И. Журавлёв, “Теоретико-множественные методы в алгебре логики”, Проблемы кибернетики, 8, Физматлит, М., 1962, 5-44.

[64] Ю.И. Журавлёв, “Алгоритм построения минимальных дизъюнктивных нормальных форм для функций алгебры логики”, Дискретная математика и математические вопросы кибернетики, Наука, М., 1974, 67-98.

[65] Ю. И. Журавлёв, “О различных понятиях минимальности д.н.ф.”, Сиб. матем. журн., 1:4 (1960), 609-610.

[66] Лин Син-лян, “О сравнении сложностей минимальных и кратчайших дизъюнктивных нормальных форм для функций алгебры логики", Проблемы кибернетики, 18, Наука, М., 1967, 11-44.

[67] В. В. Глаголев, “Оценка сложности сокращенной дизъюнктивной нормальной формы для почти всех функций алгебры логики”, Докл. АН СCCP, 158:4 (1964), 770-773; англ. пер.: V. V. Glagolev, "Estimate of the complexity of a reduced disjunctive normal form for almost all functions of the algebra of logic", Soviet Math. Dokl., 5 (1965), 1302-1305.

[68] В. В. Глаголев, "Верхняя оценка сложности минимальной д.н.ф. для почти всех функций алгебры логики", Дискретный анализ, 5, Ин-т математики СО АН СССР, Новосибирск, 1965, 3-8. 
[69] А. Д. Коршунов, "Верхняя оценка сложности кратчайших д.н.ф. почти всех булевых функций", Кибернетика, 1969, № 6, 1-8.

[70] А. А. Сапоженко, "О сложности дизъюнктивных нормальных форм, получаемых с помощью градиентного алгоритма", Дискретный анализ, 21, Ин-т математики СО АН СССР, Новосибирск, 1972, 62-71.

[71] А. Д. Коршунов, "О сложности кратчайших дизъюнктивных нормальных форм булевых функций", Методы дискретного анализа в изучении булевых функиий u графов, Методы дискретного анализа, 37, Ин-т математики СО АН СССР, Новосибирск, 1981, 9-41.

[72] А. Д. Коршунов, "О сложности кратчайших дизъюнктивных нормальных форм случайных булевых функций”, Методы дискретного анализа в оптимизации управляющих систем, Методы дискретного анализа, 40, Ин-т математики СО АН СССР, Новосибирск, 1983, 25-53.

[73] Р.Г. Нигматуллин, "Вариационный принцип в алгебре логики”, Дискретный анализ, 6, Ин-т математики СО АН СССР, Новосибирск, 1967, 69-89.

[74] А. Е. Андреев, "О синтезе дизъюнктивных нормальных форм, близких к минимальным”, Докл. АН СCCP, 269:1 (1983), 11-15; англ. пер.: A. E. Andreev, "On the synthesis of disjunctive normal forms which are close to minimal", Soviet Math. Dokl., 27 (1983), 265-269.

[75] N. Pippenger, "The shortest disjunctive normal form of a random Boolean function", Random Structures Algorithms, 22:2 (2003), 161-186.

[76] О.Б. Лупанов, "О сложности реализации функций алгебры логики релейноконтактными схемами", Проблемы кибернетики, 11, Наука, М., 1964, 25-47.

[77] О.Б. Лупанов, "О вентильных и контактно-вентильных схемах", Докл. АН СССР, 111:6 (1956), 1171-1174.

[78] Э. И. Нечипорук, "О сложности вентильных схем, реализующих булевские матрицы с неопределенными элементами", Докл. АН CCCP, 163:1 (1965), 40-42; англ. пер.: Ё. I. Nečiporuk, "Complexity of gating circuits which are realized by Boolean metrices with undetermined elements", Soviet Phys. Dokl., 10 (1966), 591-593.

[79] Э. И. Нечипорук, "О сложности схем в некоторых базисах, содержащих нетривиальные элементы с нулевыми весами”, Докл. АН СССР, 139:6 (1961), 1302-1303; англ. пер.: Eh. I. Nechiporuk, "Complexity of schemes in certain bases containing nontrivial elements with zero weights", Soviet Math. Dokl., 2 (1961), 1087-1088.

[80] А. А. Разборов, "Нижние оценки сложности реализации симметрических булевых функций контактно-вентильными схемами", Матем. заметки, 48:6 (1990), 79-90; англ. пер.: А. A. Razborov, "Lower bounds of the complexity of symmetric Boolean functions of contact-rectifier circuits", Math. Notes, 48:6 (1990), 1226-1234.

[81] D. E. Muller, "Complexity in electronic switching circuits", IRE Trans. Electron. Comput., EC-5:1 (1956), 15-19.

[82] А.Б. Угольников, "О реализации функций из замкнутых классов схемами из функциональных элементов”, Математические вопросы кибернетики, 1, Физматлит, М., 1988, 89-113.

[83] О. Б. Лупанов, "О сложности реализации степеней булевых $(n, n)$-функций”, Математические вопросы кибернетики, 12, Физматлит, М., 2003, 179-216.

[84] О.Б. Лупанов, "Об одном подходе к синтезу управляющих систем - принципе локального кодирования", Проблемы кибернетики, 14, Наука, М., 1965, 31-110.

[85] Л. А. Шоломов, "Информационные свойства функционалов сложности для систем недоопределенных булевых функций", Проблемы кибернетики, 34, Наука, M., 1978, 133-150. 
[86] Л. А. Шоломов, “О реализации недоопределенных булевых функций схемами из функциональных элементов”, Проблемы кибернетики, 21, Наука, М., 1969, $215-226$.

[87] О. Б. Лупанов, “Об одном классе схем из функциональных элементов”, Проблемы кибернетики, 7, Физматгиз, М., 1962, 61-114.

[88] Б.И. Фиников, "Об одном семействе классов функций алгебры логики и их реализации в классе П-схем", Докл. АН СССР, 115:2 (1957), 247-248.

[89] Е.П. Липатов, "Об одном случае неравномерного локального кодирования", Проблемы кибернетики, 26, Наука, М., 1973, 95-107.

[90] Е. П. Липатов, “Об асимптотически оптимальном по сложности и задержке синтезе классов вектор-функций”, Дискретный анализ, 24, Ин-т математики СО АН СССР, Новосибирск, 1974, 69-83.

[91] N. Pippenger, "Information theory and the complexity of Boolean functions", Math. Systems Theory, 10:2 (1976/77), 129-167.

[92] Л.А. Шоломов, "О функционалах, характеризующих сложность систем недоопределенных булевых функций”, Проблемы кибернетики, 19, Наука, М., 1967, $123-139$.

[93] Л.А. Шоломов, "О сложности последовательной реализации частичных булевых функций схемами", Дискрет. анализ и исслед. опер. Сер. 1, 14:1 (2007), 110-139; англ. пер.: L. A. Sholomov, "On the complexity of the sequential realization of partial Boolean functions by schemes", J. Appl. Industr. Math., 2:2 (2008), 270-289.

[94] А. А. Семенов, "Сложность приближенной реализации булевых функций схемами из функциональных элементов”, Математические вопросы кибернетики, $\mathbf{5}$, Физматлит, М., 1994, 262-279.

[95] Э. И. Нечипорук, “О синтезе схем из пороговых элементов”, Проблемы кибернетики, 11, Наука, М., 1964, 49-62.

[96] О. Б. Лупанов, “О синтезе схем из пороговых элементов”, Проблемы кибернетики, 26, Наука, М., 1973, 109-140.

[97] Н. А. Карпова, "О сложности реализации функций алгебры логики в некоторых бесконечных базисах", Методы дискретного анализа в теории булевых функuий и схем, Методы дискретного анализа, 35, Ин-т математики СО АН СССР, Новосибирск, 1980, 9-14.

[98] А. А. Марков, "Об инверсионной сложности систем функций", Докл. АН CCCP, 116:6 (1957), 917-919.

[99] А.А. Марков, "Об инверсионной сложности систем булевых функций”, Докл. АН СССР, 150:3 (1963), 477-479; англ. пер.: A. Markov, "On the inversion complexity of systems of Boolean functions", Soviet Math. Dokl., 4 (1963), 694-696.

[100] Н. А. Карпова, "О некоторых свойствах функций Шеннона", Матем. заметки, 8:5 (1970), 663-674; англ. пер.: N. A. Karpova, "Some properties of Shannon functions", Math. Notes, 8:5 (1970), 843-849.

[101] Н. А. Карпова, "Некоторые замечания об асимптотическом поведении функций Шеннона", Проблемы кибернетики, 30, Наука, М., 1975, 313-318.

[102] М. И. Гринчук, "О сложности реализации булевых функций в трех классах схем в базисе, состоящем из всех симметрических функций”, Дискрет. анализ и исслед. опер., 3:1 (1996), 3-8.

[103] О.М. Касим-Заде, "Об одном методе получения оценок сложности над бесконечными базисами", Математические вопросы кибернетики, 11, Физматлит, М., 2002, 247-254.

[104] О.М. Касим-Заде, "Об одном методе получения оценок сложности схем над произвольным бесконечным базисом", Дискрет. анализ и исслед. опер. Сер. 1, 11:2 (2004), 41-65. 
[105] Н. А. Карпова, "О сложности класса схем из многополюсных функциональных элементов", Математические вопросы кибернетики, 7, Физматлит, М., 1998, 67-84.

[106] Н. А. Карпова, "О сложности класса схем из информационно бедных многополюсных элементов", Математические вопросы кибернетики, 15, Физматлит, М., 2006, 155-164.

[107] С. Б. Гашков, "О глубине булевых функций”, Проблемы кибернетики, 34, Наука, M., 1978, 265-268.

[108] О. М. Касим-Заде, “О глубине булевых функций при реализации схемами над произвольным базисом", Вестн. Моск. ун-та. Сер. 1. Матем., мех., 2007, № 1, 18-21; англ. пер.: O. M. Kasim-Zade, "The depth of the Boolean functions in the realization by the schemes over an arbitrary basis", Moscow Univ. Math. Bull., 62:1 (2007), 18-21.

[109] О. Б. Лупанов, "О схемах из функциональных элементов с задержками”, Проблемы кибернетики, 23, Наука, М., 1970, 43-81.

[110] В. М. Храпченко, "Различие и сходство между задержкой и глубиной", Проблемы кибернетики, 35, Наука, М., 1979, 141-168.

[111] В.М. Храпченко, "Новые соотношения между глубиной и задержкой", Дискрет. матем., 7:4 (1995), 77-85; англ. пер.: V. M. Khrapchenko, "New inequality relations between depth and delay", Discrete Math. Appl., 5:6 (1995), 547-555.

[112] В.М. Храпченко, "Принципиальное расхождение между глубиной и задержкой", Дискрет. матем., 20:3 (2008), 51-72; англ. пер.: V. M. Khrapchenko, "The fundamental difference between depth and delay", Discrete Math. Appl., 18:4 (2008), 391-412.

[113] М.Н. Вайнцвайг, "О мощности схем из функциональных элементов”, Докл. АН CCCP, 139:2 (1961), 320-323; англ. пер.: M. Vaincvaig, "On the power of networks of functional elements", Soviet Phys. Dokl., 6 (1962), 454-547.

[114] О.М. Касим-Заде, "Об одновременной минимизации сложности и мощности схем из функциональных элементов", Проблемы кибернетики, 33, Наука, М., 1978, 215-220.

[115] О. М. Касим-Заде, "Об одной мере сложности схем из функциональных элементов", Проблемы кибернетики, 38, Наука, М., 1981, 117-179.

[116] О. М. Касим-Заде, "Об одной мере активности схем из функциональных элементов”, Математические вопросы кибернетики, 4, Физматлит, М., 1992, 218-228.

[117] А. В. Кузнецов, "О средствах для обнаружения невыводимости или невыразимости", Логический вывод, Наука, М., 1979, 5-53.

[118] О.М. Касим-Заде, "О сложности параметрических представлений булевых функций”, Математические вопросы кибернетики, 7, Физматлит, М., 1998, $85-160$.

[119] А. Д. Коршунов, “Об оценках сложности схем из объемных функциональных элементов и объемных схем из функциональных элементов", Проблемы кибернетики, 19, Наука, М., 1967, 275-283.

[120] С. С. Кравцов, "О реализации функций алгебры логики в одном классе схем из функциональных и коммутационных элементов", Проблемы кибернетики, 19, Наука, М., 1967, 285-292.

[121] А. Альбрехт, "О схемах из клеточных автоматов", Проблемы кибернетики, 33, Наука, М., 1978, 209-214.

[122] Н. А. Шкаликова, "О сложности реализации некоторых функций клеточными автоматами", Сб. работ по математической кибернетике, 1, ВЦ АН СССР, М., 1967, 102-115. 
[123] Э. И. Нечипорук, "О сложности схем в некоторых базисах, содержащих нетривиальные элементы с нулевыми весами", Проблемы кибернетики, 8, Физматгиз, M., 1962, 123-160.

[124] Е. С. Горелик, "О сложности реализации элементарных конъюнкций и дизъюнкций в базисе $\{x / y\}$ ”, Проблемы кибернетики, 26, Наука, М., 1973, 27-36.

[125] Г. А. Кочергина, "О сложности реализации элементарных конъюнкций и дизъюнкций схемами в некоторых полных базисах", Математические вопросы кибернетики, 11, Физматлит, М., 2002, 219-246.

[126] C. P. Schnorr, "The combinational complexity of equivalence", Theoret. Comput. Sci., 1:4 (1976), 289-295; рус. пер.: К. П. Шнорр, "Комбинационная сложность эквивалентности", Кибернет. сб. Нов. сер., 16, Мир, М., 1979, 74-81.

[127] Н. П. Редькин, “Доказательство минимальности некоторых схем из функциональных элементов”, Проблемы кибернетики, 23, Наука, М., 1970, 83-101.

[128] Н. П. Редькин, "О минимальной реализации двоичного сумматора", Проблемы кибернетики, 38, Наука, М., 1981, 181-216.

[129] И. С. Шкребела, "О сложности реализации линейных булевых функций схемами из функциональных элементов в базисе $\{x \rightarrow y, \bar{x}\}$ ", Дискрет. матем., 15:4 (2003), 100-112; англ. пер.: I. S. Shkrebela, "On complexity of realisation of linear Boolean functions by circuits of functional elements over the basis $\{x \rightarrow y, \bar{x}\}$ ", Discrete Math. Appl., 13:5 (2003), 483-496.

[130] Э. И. Нечипорук, "Об одной булевской матрице”, Проблемы кибернетики, 21, Наука, М., 1969, 237-240.

[131] L. H. Harper, "An $n \log n$ lower bound on synchronous combinational complexity", Proc. Amer. Math. Soc., 64:2 (1977), 300-306.

[132] L. H. Harper, J. E. Savage, "Lower bounds on synchronous combinational complexity", SIAM J. Comput., 8:2 (1979), 115-119.

[133] Л. А. Шоломов, "Об информационной сложности задач, связанных с минимальной реализацией булевых функций схемами", Проблемы кибернетики, 26, Наука, М., 1973, 207-256.

[134] Л. А. Шоломов, “Об одной последовательности сложно реализуемых функций”, Матем. заметки, 17:6 (1975), 957-966; англ. пер.: L. A. Sholomov, "A sequence of complexly computable functions", Math. Notes, 17:6 (1975), 574-579.

[135] С. С. Марченков, "О сложности вычисления экспоненты”, Матем. заметки, 31:3 (1982), 457-463; англ. пер.: S. S. Marchenkov, "The complexity of computing exponents", Math. Notes, 31:3 (1982), 234-237.

[136] А. А. Разборов, "Нижние оценки монотонной сложности логического перманента", Матем. заметки, 37:6 (1985), 887-900; англ. пер.: А. A. Razborov, "Lower bounds on monotone complexity of the logical permanent", Math. Notes, 37:6 (1985), 485-493.

[137] А.Е. Андреев, "Об одном методе получения эффективных нижних оценок монотонной сложности", Алгебра и логика, 26:1 (1987), 3-26; англ. пер.: A.E. Andreev, "A method for obtaining efficient lower bounds for monotone complexity", Algebra Logic, 26:1 (1987), 1-18.

[138] J. E. Hopcroft, R. M. Karp, "An $n^{5 / 2}$ algorithm for maximun matchings in bipartite graphs", SIAM J. Comput., 2:4 (1973), 225-231.

[139] L. Lovász, "The works of A. A. Razborov", Proceedings of the International Congress of Mathematicians, v. I (Kyoto, 1990), Math. Soc. Japan, Tokyo, 1991, 37-40; pyc. пер.: Л. Ловас, "О работах А. А. Разборова", Международный конгресс математиков (Киото, 1990), Мир, М., 1996, 52-58.

[140] А.А. Разборов, "Нижние оценки сложности булевых функций”, Proceedings of the International Congress of Mathematicians, v. 2 (Berkeley, CA, 1986), Amer. 
Math. Soc., Providence, RI, 1987, 1478-1487; англ. пер.: A. A. Razborov, "Lower bounds for monotone complexity of Boolean functions", Amer. Math. Soc. Trans. (2), 147 (1990), 75-84.

[141] N. Alon, R. B. Boppana, "The monotone circuit complexity of Boolean functions", Combinatorica, 7:1 (1987), 1-22.

[142] А. Карацуба, Ю. Офман, "Умножение многозначных чисел", Докл. АН СССР, 145:2 (1962), 293-294.

[143] А. Л. Тоом, "О сложности схемы из функциональных элементов, реализующей умножение целых чисел", Докл. АН СССР, 150:3 (1963), 496-498.

[144] A. Schönhage, V. Strassen, "Schnelle Multiplikation großer Zahlen", Computing, 7:3-4 (1971), 281-292; рус. пер.: А. Шёнхаге, В. Штрассен, "Быстрое умножение больших чисел", Кибернет. сб. Нов. сер., 10, Мир, М., 1973, 87-98.

[145] А. Л. Тоом, "О сложности реализации двоичных функций, имеющих мало подфункций”, Проблемы кибернетики, 18, Наука, М., 1967, 83-90.

[146] Д. Улиг, "О связи между сложностью схемной реализации функций алгебры логики и числом их подфункций", Проблемы кибернетики, 26, Наука, М., 1973, $183-201$.

[147] Д. Улиг, "Об одном семействе классов просто реализуемых функций алгебры логики", Проблемы кибернетики, 28, Наука, М., 1974, 25-42.

[148] В. М. Храпченко, "Об асимптотической оценке времени сложения параллельного сумматора", Проблемы кибернетики, 19, Наука, М., 1967, 101-122.

[149] Е. Ю. Захарова, "Об одном обобщении электронно-ламповых схем", Проблемы кибернетики, 7, Физматлит, М., 1962, 43-59.

[150] Б. А. Субботовская (Мучник), "О сравнении базисов при реализации функций алгебры логики формулами", Докл. АН СCCP, 149:4 (1963), 784-787; англ. пер.: В. A. Subbotovskaya, "Comparison of bases in the realization by formulas of functions of the algebra of logic", Soviet Math. Dokl., 4 (1963), 478-481.

[151] Б. А. Мучник (Субботовская), "Об одном критерии сравнимости базисов при реализации функций алгебры логики формулами", Матем. заметки, 1:5 (1967), 515-524; англ. пер.: В. А. Muchnik, "One criterion for comparability of bases for the realization of Boolean functions by formulas", Math. Notes, 1:5 (1967), 341-346.

[152] Д. Ю. Черухин, "Об одной бесконечной последовательности улучшающихся булевых базисов”, Дискрет. анализ и исслед. опер. Сер. 1, 4:4 (1997), 79-95.

[153] Д. Ю. Черухин, "О сложности реализации линейной функции формулами в конечных булевых базисах", Дискрет. матем., 12:1 (2000), 135-144; англ. пер.: D. Yu. Cherukhin, "On the complexity of realization of the linear function by formulas over finite Boolean bases", Discrete Math. Appl., 10:2 (2000), 147-157.

[154] Д. Ю. Черухин, "О реализации линейной функции формулами в различных базисах", Вестн. Моск. ун-та. Сер. 1. Матем., мех., 2001, №6, 15-19; англ. пер.: D. Yu. Cherukhin, "Realization of linear functions by formulas in various bases", Moscow Univ. Math. Bull., 56:6 (2001), 14-18.

[155] Р.Е. Кричевский, "О реализации функций суперпозициями”, Проблемы кибернетики, 2, Физматгиз, М., 1959, 123-138.

[156] А.Е. Андреев, "Об одном методе синтеза формул", Вестн. Моск. ун-та. Сер. 1. Матем., мех., 1994, №6, 23-27; англ. пер.: А.Е. Andreev, "On a formula synthesizing method", Moscow Univ. Math. Bull., 49:6 (1994), 22-25.

[157] Н. А. Карпова, "О возможном асимптотическом поведении функций Шеннона при реализации функций алгебры логики формулами", Проблемы кибернетики, 26, Наука, М., 1973, 37-51.

[158] Э. И. Нечипорук, "О синтезе логических сетей в неполных и вырожденных базисах", Проблемы кибернетики, 14, Наука, М., 1965, 111-160. 
[159] В. М. Храпченко, "О соотношении между сложностью и глубиной формул", $M e-$ тоды дискретного анализа в синтезе управляющих систем, Методы дискретного анализа, 32, Ин-т математики СО АН СССР, Новосибирск, 1978, 76-94.

[160] С. А. Ложкин, "О синтезе формул, сложность и глубина которых не превосходят асимптотически наилучших оценок высокой степени точности", Becтн. Моск. ун-та. Сер. 1. Матем., мех., 2007, № 3, 19-25; англ. пер.: S. A. Lozhkin, "Synthesis of formulas whose depth and complexity do not exceed asymptotically the best estimates of high accuracy", Moscow Univ. Math. Bull., 62:3 (2007), 101-107.

[161] О.Б. Лупанов, "О влиянии глубины формул на их сложность", Кибернетика, 1970, № 2, 46-49.

[162] M. J. Fischer, A. R. Meyer, M. S. Paterson, "Lower bounds on the size of Boolean formulas: preliminary report", Seventh Annual ACM Symposium on Theory of Computing (Albuquerque, NM, 1975), Assoc. Comput. Mach., New York, 1975, 37-44.

[163] Д. Ю. Черухин, "Нижние оценки формульной сложности симметрических булевых функций”, Дискрет. анализ и исслед. опер. Сер. 1, 7:3 (2000), 86-98.

[164] Б. А. Мучник (Субботовская), “Оценка сложности реализации линейной функции формулами в некоторых базисах", Кибернетика, 1970, № 4, 29-38.

[165] В. М. Храпченко, "Об одном методе получения нижних оценок сложности П-схем", Матем. заметки, 10:1 (1971), 83-92; англ. пер.: V. M. Khrapchenko, "Method of determining lower bounds for the complexity of P-schemes", Math. Notes, 10:1 (1971), 474-479.

А. Д. Коршунов (A.D. Korshunov)

Институт математики им. С. Л. Соболева СО РАН

E-mail: korshun@math.nsc.ru
Поступила в редакцию 04.10 .2011 